STRATEGIES FOR THE SUST AINABLE USE AND MANAGEMENT OF TIMBER TREE SPECIES SUBJECT TO INTERNATIONAL TRADE:

\title{
SOUTH EAST ASIA
}

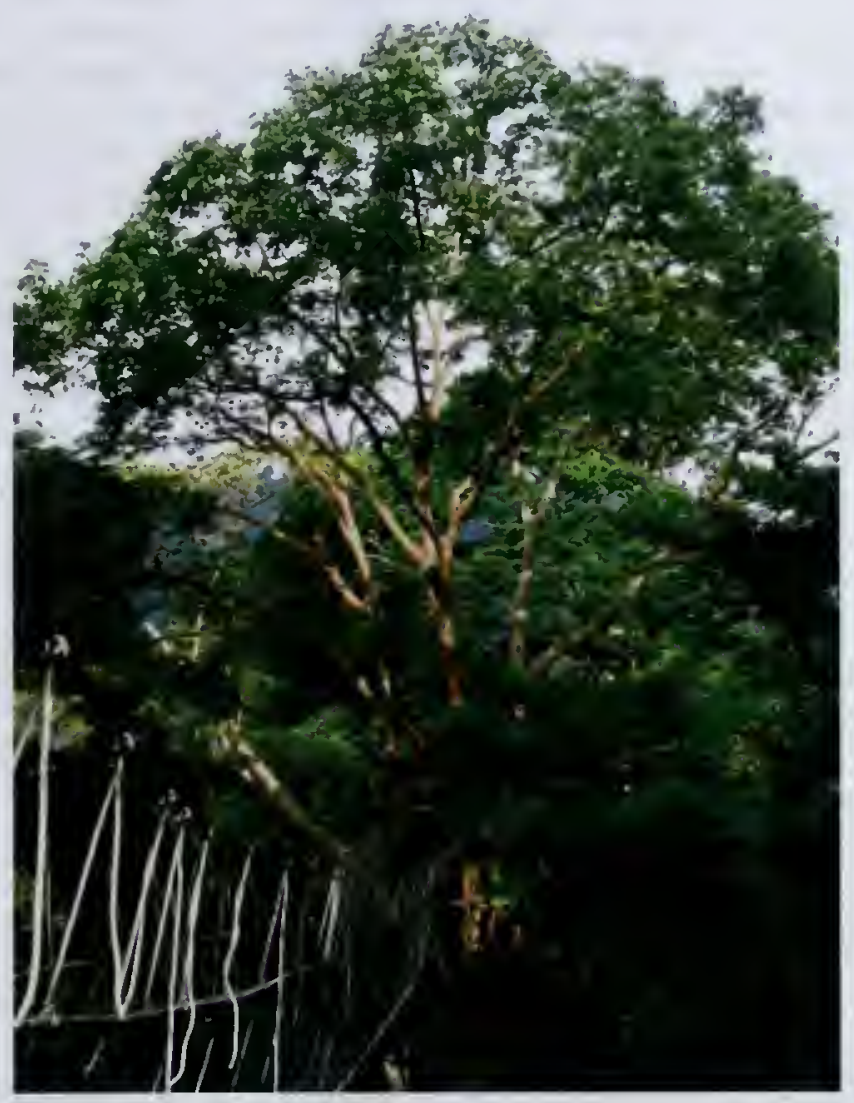

2008

COMPILED BY UNEP-WCMC

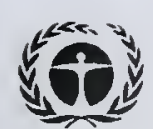

UNEP WCMC

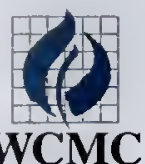

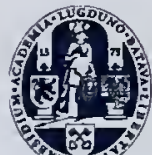

Universiteit Leiden

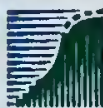


Prepared and produced by: UNEP World Conservation Monitoring Centre, Cambridge, UK

\section{About UNEP World Conservation Monitoring Centre}

www.unep-wcmc.org

The UNEP World Conservation Monitoring Centre is the biodiversity assessment and policy implementation arm of the United Nations Environment Programme (UNEP), the world's foremost intergovernmental environmental organisation. UNEP-WCMC aims to help decision-makers recognize the value of biodiversity to people everywhere, and to apply this knowledge to all that they do. The Centre's challenge is to transform complex data into policy-relevant information, to build tools and systems for analysis and integration, and to support the needs of nations and the international community as they engage in joint programmes of action.

UNEP-WCMC provides objective, scientifically rigorous products and services that include ecosystem assessments, support for implementation of environmental agreements, regional and global biodiversity information, research on threats and impacts, and development of future scenarios for the living world.

Prepared by UNEP-WCMC, with the support of the governments of the Netherlands and the United Kingdom of Great Britain and Northern Ireland.

Edited by: Harriet Gillett \& Pablo Sinovas, UNEP-WCMC

(C) Copyright: UNEP World Conservation Monitoring Centre, 2008

The designations of geographical entities in this report and the presentation of the material, do not imply the expression of any opinion whatsoever on the part of UNEPWCMC concerning the legal status of any country, territory, or area, or of its authorities, or concerning the delimitation of its frontiers or boundaries.

Cover photo: Canopy walkway, supported by Intsia palembanica (Merbau), Bukit Lagong Forest Reserve, Forest Research. Institute of Malaysia $\mathbb{C}$ Harriet Gillett 
Forests 3

Forest Protection: national \& international actions ............................................... 4

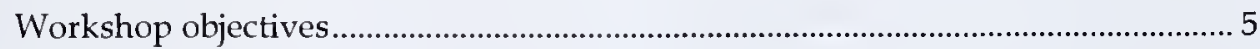

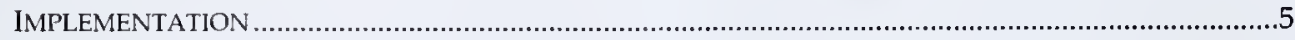

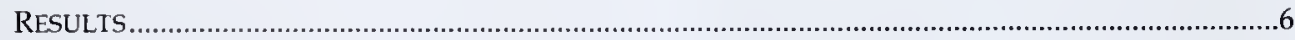

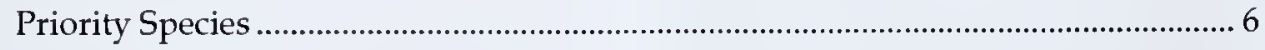

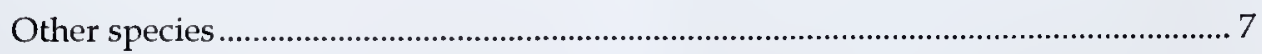

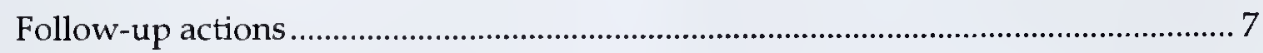

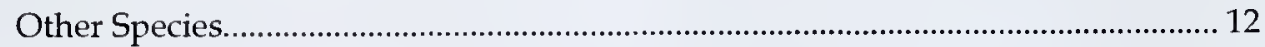

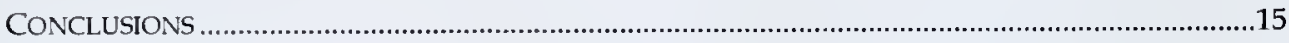

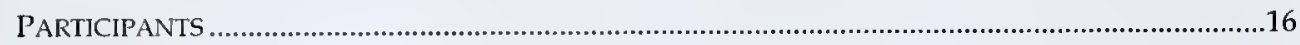

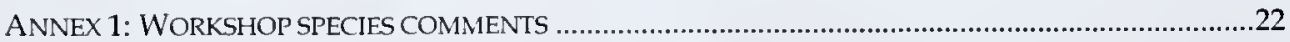

Table 1. Timber tree species in international trade, of conservation concern.......... 22

Table 2. Timber tree species in international trade, of possible conservation

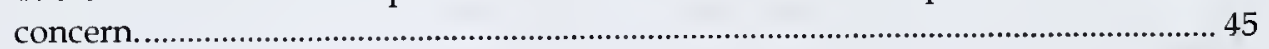

Table 3. Timber tree species possibly in international trade and of conservation concern, but in need of taxonomic revision................................................................ 51

Table 4. Timber tree species in international trade, not considered to be of

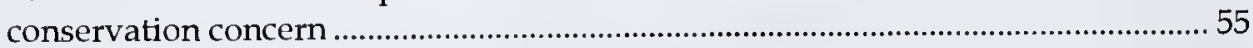

Table 5. Timber tree of conservation concern not thought to be in international

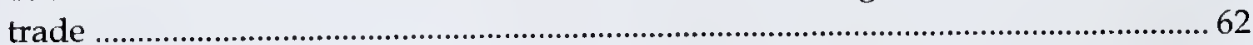

Table 6. Timber tree species not thought to be in international trade or to be of conservation concern 68 


\section{ACKNOWLEDGEMENTS}

The participants at the workshop were fundamental to the success of the project, sharing their extensive knowledge and experience before, during and after the workshop. The project benefited from the enthusiastic collaboration of all present. They, and the organisations supporting their attendance at the workshop are thanked for their contributions. Jan de Koning (the Netherlands) and Greg Leach (Australia), jointly chaired the workshop, and were critical to ensuring rapid progress was made and conclusions agreed for each of the 115 species under discussion.

The project was managed by Harriet Gillett. Pablo Sinovas acted as rapporteur and provided logistical support to the workshop. Soh Wuu Kuang updated the background documentation, including the incorporation of data on new species, when he was a UNEP-WCMC Chevening Scholar in Biodiversity
2005/2006, funded by the United Kingdom (Foreign \& Commonwealth Office) and the Salters' Company. He also helped organise and facilitate the workshop. Gerardo Fragoso helped guide project development and implementation and was responsible for overall project supervision.

The workshop was funded by the governments of the Netherlands (Ministry of Agriculture, Nature and Food Quality) and the United Kingdom (Defra). The support from and collaboration of Stefan Verbunt, Chris Schürmann and Jan de Koning (the Netherlands) is gratefully acknowledged. James Paver (United Kingdom) is also thanked for his support at the workshop.

Dato' Dr. Wan Razali, the Deputy Director General of FRIM is thanked for providing an official welcome on behalf of the Ministry of Natural Resources and Environment.

\section{ORGANISATIONS, ACRONYMS AND WEBLINKS}

CBD

CITES

Defra

FAO

FFI

FRIM

TRAFFIC

UNEP

UNEP-WCMC
Convention on Biological Diversity www.biodiv.org

Convention on International Trade in Endangered Species of Fauna and Flora www.cites.org

Department for Environment, Food and Rural Affairs www.defra.gov.uk

Food And Agriculture Organization Of The United Nations

www.fao.org

Fauna and Flora International www fauma-flora.org

Forest Research Institute of Malaysia www.frim.gov.mv

TRAFFIC International www. traffic.org

United Nations Environment Programme

http://www.unep.org

United Nations Environment Programme World Conservation Monitoring Centre http:/ / www.unep-wcmc.org

World Wide Fund for Nature http://www. wwf.org 
Internationally agreed targets to manage the world's living resources sustainably are recognised by the United Nations (Millenium Development Goal 7), Convention on Biological Diversity (CBD; 2010 target), and CITES (Strategic Plan). Timber producing trees are particularly prone to overexploitation. For species in international trade, regionally agreed sustainable use and management strategies are needed at the species level if these international targets are to be met.

South East Asian timber tree species in international trade and in need of action at the national or international level to ensure their long-term sustainable use, were identified by a panel of experts at a regional workshop held in Kuala Lumpur, Malaysia, 5-7 September 2007.
The group involved experts from various sectors: forestry, timber trade, plant taxonomy, botanic garden, CITES and FAO. Experiences in the management and sustainable trade of a total of 122 regional timber species were shared to identify species that are or that may be subject to unsustainable harvesting for international trade. Details were collated on the pressures or threats that the species' face, including knowledge relevant to the use of each species.

Sixty-six species were considered to be in international trade and of known, or possible, conservation concern (tables 1, 1A \& 2). Of these, 15 species and the genera Dalbergia and Diospyros (included in tables $1 \& 1 \mathrm{~A}$ ) were identified as immediate priorities for action at the national or international level.

\section{INTRODUCTION}

\section{Forests}

Forests play a critical role in the lives and wellbeing of people around the world. Six million indigenous people living in the rain forests of Latin America, Southeast Asia and West Africa depend heavily on forests. A further 350 million people living in, or next to, dense forests rely on them for subsistence or income whilst 1.2 billion people in developing countries use trees on farms to generate food and cash (FAO, 2005'1).

Forests also play a major role in relation to climate change. According to FAO (2005) they contribute about one-fifth of global carbon emissions

\footnotetext{
1 FAO. 2005. FAO website www.fao.org/forestry
}

when cleared; they react sensitively to a changing climate; when managed sustainably, they produce wood fuels as a benign alternative to fossil fuels; and finally, they have the potential to absorb about one-tenth of projected global carbon emissions into their biomass, soils and products and store them - in principle in perpetuity.

Despite this, the world's natural forests are shrinking and forest systems worldwide are under threat for a wide range of reasons. These include habitat destruction, and exploitation for use as timber, firewood or for other non-timber forest products, which are major commodities in international trade. However, whereas habitat destruction may impact the forest in general, use, 
and particularly international use, of trees is species specific, depending on the quality of the product used: wood, fruit, sap etc. The very high value of a final product may put great pressure on the species concerned leading to the decline of high value tree species in their natural habitats. However, in general, efforts to ensure the sustainable use of forests have so far focussed on the forest as a whole rather than on particular species that may be subject to specific exploitation.

\section{Forest Protection: national \& international actions}

Countries have addressed the issue of forest protection through tools such as legally gazetting areas in which disturbance to the ecosystem is then limited, and by passing legislation that limits the species and the size and quantity of specimens that may be felled or harvested. However, there is evidence of low levels of compliance of both protected area and timber harvesting legislation, and international trade in illegally sourced timber is a major source of concern. International efforts have therefore developed to address two issues: 1) ensuring forest use is sustainable; 2) ensuring forest use is legal.

Direct measures to manage the way in which trees are exploited include development of certification standards and standards that codify practices for sustainable forestry. These are essentially voluntary and depend on a range of factors, including producer engagement; effective monitoring of the frequently complex chain-ofcustody from producer to consumer; and consumer demand.

International initiatives with targets that encompass or necessitate safeguarding the world's forests include:

UN Millennium Development Goal 1 to eradicate extreme poverty and hunger
> UN Millennium Development Goal 7 to ensure environmental sustainability

$>$ CBD 2010 target, "to achieve by 2010 a significant reduction of the current rate of biodiversity loss at the global, regional and national level" and, CBD Global Strategy for Plant Conservation

$>$ CITES strategic plan: No species of wild flora subject to unsustainable exploitation because of international trade

Relevant actions that need to be implemented to ensure sustainable forest management are identified in the World Summit on Sustainable Development (WSSD) Plan of Implementation and the CBD Workplan. These include the need to:

$>$ Determine status and conservation needs of endemic or threatened species and the impacts of current forest management practices on them;

$>$ Develop initiatives that address the sustainable use of timber and non-timber forest products;

- Assist importing countries to prevent the entry of unsustainably harvested forest resources which are not covered by CITES. 
The workshop directly addressed these three needs. Demand for forest information has never been greater or more complex than now, with countries being asked to report regularly in order to fulfil their obligations to the international agreements to which they are party, and as civil society becomes increasingly concerned about the state and trends in forest stocks, goods and services.

The current initiative was undertaken in response to the need to ensure that authoritative information at the species level is available to those people responsible for decision making. Thus, the workshop's specific objective was to identify, on a regional basis, timber tree species involved in international trade, for which data on use and population size indicate that species specific management plans are needed, and additionally to produce recommendations for these species. By collaborating with organisations at a regional level, synthesising all existing relevant information and making this available in an integrated format, the capacity of countries and organisations to make well-informed decisions is enhanced, particularly for species occurring in more than one country in the region.

\section{IMPLEMENTATION}

The concept for this workshop was based on earlier work funded by the governments of the Netherlands and the United Kingdom. This earlier work included compilation of a desk study Contribution to an evaluation of tree species using the new CITES listing criteria in 1998 (funded by the Netherlands). A history of subsequent activities leading up to the workshop is provided on the CITES website in PC16 Doc. 19.22

This work lead to the decision to implement a series of regional workshops to bring regional experts together to discuss the timber trees of each region included in the desk study. The objective was to identify species in international trade and of conservation concern and to subsequently identify strategies to support their long-term sustainable use.

2

http://www.cites.org/eng/com/PC/16/

E-PC16-19-02.pdf
The first regional workshop, for Central America, was held in Nicaragua in 2005.

Experts from South East Asian countries were invited to participate in a regional workshop, held in Malaysia, 5-7 September 2007. In addition, representatives of UN organisations, other inter-governmental organisations, and non-governmental organisations were invited to attend. Participants had diverse backgrounds and experience of the forest sector, timber trade, plant taxonomy, and implementation of CITES.

Participants were asked to provide information on 115 South East Asian timber tree species in international trade, identified by UNEP-WCMC based on a global survey undertaken previously (WCMC, 1999). The background information on the 115 species is available at:

www.unep-wcmc.org/forest/timber 
The participants discussed the 115 species, with a view to identifying, as a priority, those that are in international trade and of known or of possible conservation concern.
Participants also nominated an additional seven species for consideration and provided information on actions currently underway in their countries in relation to all the species discussed.

\section{RESULTS}

The 115 timber species discussed in detail during the workshop were eventually divided into six categories, according to the criteria:

1 In intemational trade $\mathcal{E}$ threatened (Table 1)

- Threatened in at least one country/province in the region (excluding Singapore, which was considered together with Peninsular Malaysia).

- Known to be threatened, but threat not necessarily due to trade.

$2 \quad$ In international trade but data deficient for conservation concem (Table 2).

- Threatened in at least one country/province in the region (excluding Singapore, which was considered together with Peninsular Malaysia).

- Insufficient information available to determine whether the species is threatened

\section{Taxononic confusion (Table 3)}

- Possibly in intemational trade and possibly of conservation concern.

- Confusion over taxonomy of the species prevents clear understanding of the trade situation.

4 In international trade, but not considered to be threatened (Table 4).

- Known to be in international trade.

- Available information suggests the species is not threatened.
$5 \quad$ Not in international trade but thought to be threatened by workshop participants or previously recorded as threatened by IUCN (Table 5).

- Not known to be in international trade.

- Available information suggests the species is threatened.

6 Not in international trade $\mathcal{E}$ not considered to be threatened (Table 6).

- Not known to be in international trade.

- Not known to be threatened.

Due to the large number of species for discussion, the decision was taken to focus the workshop on considering data on the status of the 115 species.

It was agreed that attempting to identify relevant actions for each species was impracticable within the time constraints. Information on the 115 species provided by workshop participants is given in Annex 1 (Tables 1-6).

This information augments the data provided in the background documentation, available at www.unep-rwcmc.org/forest/timber

\section{Priority Species}

Fifty-nine of the 115 species were identified as being in international trade, and of conservation concern, or possibly of conservation concern.

Forty species (Table 1) were identified as being in international trade and of conservation concern and 19 species 
(Table 2) were identified as being in international trade and of possible conservation concern.

Eight species and two genera in table 1 were identified by particular countries as being of highest priority for action.

Seven additional species identified by participants as being of conservation concern and in international trade and considered to be priorities for action are listed in table $1 \mathrm{~A}$. There was insufficient time and information to discuss these in detail.

\section{Other species}

Seven species (Table 3) were considered to be subject to taxonomic confusion, but possibly in international trade and possibly of conservation concern.

Seventeen species were considered to be in international trade, but not threatened (table 4). Thirty two species were considered not to be in international trade (tables 5 and 6).

\section{Follow-up actions}

Actions proposed by the workshop participants for the species identified as priority by specific countries are included for each of those species in the Annex.
A number of other actions were proposed during the workshop. These are detailed in the Annex and are also outlined below:

a. The background document for Neesia malayana indicating that the species is threatened in Indonesia was questioned, and it was suggested that Indonesia undertake research to assess status.

b. It was decided that ITTO would be asked to check the nomenclature of timber traded as Slorea in order to avoid confusion between species in trade statistics.

c. Dalbergia bariensis is under revision in Thailand, and it was noted that one of the Thai participants (Dr. Chayamarit) could supply information on who is carrying out the revision, so that the botanists involved can be contacted.

d. Further clarification was considered to be needed for Palaquium bataanense, and it was suggested that UNEP-WCMC and the Philippines double-check on which list it should be included. 
Table 1: Timber tree species in international trade, of conservation concern: priorities for action ${ }^{3}$

$$
\text { Family Species }
$$

1 Anacardiaceae Mangifera macrocarpa

2 Apocynaceae Dyera polypliylla

3 Bombacaceae Neesia altissinn

$4 \quad$ Neesia malayana

5 Burseraceae Canarium

pseudosmmatranumu

6 Crypteroniaceae $\begin{aligned} & \text { Dactylocladus } \\ & \text { stenostacliys }\end{aligned}$

7 Dipterocarpaceae Anisoptern costata

8

9

10

11

12

13 Ebenaceae

\begin{tabular}{lllll}
\hline 14 & Diospyros plilippinensis & $\underline{\mathrm{G}}$ & $\underline{\mathrm{G}}$ & \\
\cline { 2 - 5 } 15 lcacinaceae & Cantleya cormiculata & $\sqrt{ }$ & $\sqrt{ }$ & \\
\hline 16 Lauraceae & Eusideroxylon zungeri & $\sqrt{ }$ & $\underline{\mathrm{S}}$ & $\sqrt{ }$
\end{tabular}

${ }^{3} \underline{S}$ indicates species identified by that range state as a priority.

$\underline{G}$ indicates the species is from a genus identified as a priority

$\sqrt{ }$ species occurrence confirmed

$(\sqrt{ })$ species occurrence uncertain

${ }^{4}$ Known occurrence in the countries participating in the workshop (except for Singapore).

Cambodia KH

Indonesia ID

Lao P.D.R. LA

Malaysia $\quad$ MY

Philippines $\mathrm{PH}$

Thailand TH

Viet Nam VN

5 "Other" refers to occurrence in any of the remaining SE Asian countries, in particular Brunei, Myanmar and/or Singapore. 
Family

Species

Afzelia xylocarpa

18

19

20

21

22

23

24

25

26

27

28

29

30

31

32

33

34

35

36

37 Pinaceae

38 Proteaceae

39 Sapotaceae

40

Cymonetra elmeri

Dalbergia annamensis

Dalbergia cambodiana

Dalhergia mammosa

Dalhergia olizeri

Dalbergia tonkinensis

Erythroplileum fordii

Intsia bijnga

Koompassia excelsa

Sindora inermis

Sindora supa

Xylia xylocarpa

Pinns merkusii

Madluca pasquieri

Cynometra inaequifolia

Cynometra malaccensis

[Also listed in table 3]

Dalhergia cochinchinensis

Dialium cochinchinense

Koompassia grandiflora

Koompassia malaccensis

Pterocarpus macrocarpus

Alloxylon brachycarpum

$\underline{G}$

$\underline{G}$

KH ID LAMYPHTH VNOther

$\checkmark$

$\checkmark$

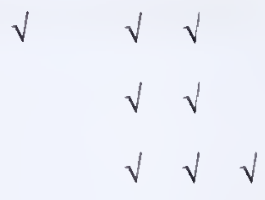

$\underline{G}$

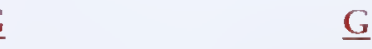

$\underline{G} \quad \underline{G} \quad \underline{G}$

$\underline{G}$

$\underline{G} \quad \underline{G} \quad \underline{G} \quad \underline{G}$

$\underline{\mathrm{S}}$

$(\sqrt{)}$

$\sqrt{ }$

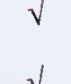

$\sqrt{ } \sqrt{ } \sqrt{ } \quad \sqrt{ }$

S $\sqrt{ } \sqrt{ } \quad \sqrt{ }$

$\sqrt{ }$
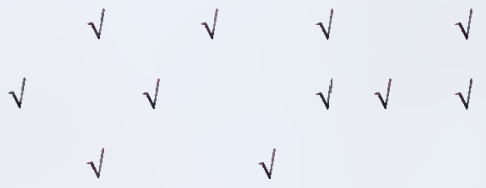

G

G 
Table 1A: Additional timber tree species in international trade, of conservation concern, identified by workshop participants as priorities for action
Species
Proposed by
Workshop comments

Bignoniaceae

1 Markhamia

Viet Nam The VN participants proposed carrying out stipulata further research on the species' ecology, distribution, uses, planting and conservation status.

Dipterocarpaceae

2 Parashorea

Viet Nam

See above.

chinensis

\section{Guttiferae}

3 Mesua ferrea

Viet Nam

See above

\section{Lauraceae}

4 Potoxylon melagangai
Malaysia

MY participants noted that felling restrictions for the species are in place in the country.

Also, see comments under Dactylocladus stenostachys (Annex 1, Table 1, record 6).

Endemic to Borneo.

\section{Leguminosae}

5 Intsia palembanica Indonesia \& Malaysia

For ID participants' comments see Shorea curtisii Annex Table 1 (record 10).

MY participants noted that felling restrictions for the species are in place in the country. Also, see comments under Dactylocladus stenostachys (Annex 1, Table 1, record 6).

\section{Leguminosae}

\section{Sindora siantensis Viet Nam}

VN participants proposed carrying out further research on the species' ecology, distribution, uses, planting and conservation status.

Meliaceae 
Table 2: Timber tree species in international trade, data deficient for conservation concern

\begin{tabular}{|c|c|c|c|c|c|c|c|c|}
\hline \multirow{2}{*}{ Family } & \multirow[t]{2}{*}{ Species } & \multicolumn{7}{|c|}{ Range States } \\
\hline & & KH & ID & LA & MY & PH & TH VN & Other \\
\hline 1 Celastraceae & Loplopetalum javanicum & & $\sqrt{ }$ & & $\sqrt{ }$ & $\sqrt{ }$ & $\sqrt{ }$ & \\
\hline 2 & Loplopetalum multinervium & & $\sqrt{ }$ & & $\sqrt{ }$ & & & $\sqrt{ }$ \\
\hline 3 & Loplopetalım pachypluyllum & & $\sqrt{ }$ & & $\sqrt{ }$ & & & \\
\hline 4 & Lopinopetallum rigidnm & & $\sqrt{ }$ & & $\sqrt{ }$ & & & $\sqrt{ }$ \\
\hline 5 Chrysobalanaceae & Parinari costata & & $\sqrt{ }$ & & $\sqrt{ }$ & $\sqrt{ }$ & & $\sqrt{ }$ \\
\hline 6 & Parinari oblongifolia & & $\sqrt{ }$ & & $\sqrt{ }$ & & & $\sqrt{ }$ \\
\hline 7 Ebenaceae & Diospyros rumpliii & & $\sqrt{ }$ & & & & & \\
\hline 8 Flacourtiaceae & Hydnocarpus sumatrana & & $\sqrt{ }$ & & $\sqrt{ }$ & $\sqrt{ }$ & $\sqrt{ }$ & \\
\hline 9 Guttiferae & Calopluyllum cannum & & $\sqrt{ }$ & & $\sqrt{ }$ & & & $\sqrt{ }$ \\
\hline 10 & $\begin{array}{l}\text { Calophyllum carrii var. } \\
\text { longigemmatum }\end{array}$ & & $\sqrt{ }$ & & & & & \\
\hline 11 & Calopliyllum insularum & & $\sqrt{ }$ & & & & & \\
\hline 12 & Calopinyllum papuanum & & $\sqrt{ }$ & & & & & \\
\hline 13 Leguminosae & Albizia splendens & & $\sqrt{ }$ & & $\sqrt{ }$ & & $\sqrt{ }$ & $\sqrt{ }$ \\
\hline$\overline{14}$ Myrtaceae & Syzygium flosculifera & & & & $\sqrt{ }$ & & & \\
\hline 15 & Syzygium koordersiana & & & & $\sqrt{ }$ & & & \\
\hline 16 & Syzygium ridleyi & & & & $\sqrt{ }$ & & $\sqrt{ }$ & $\sqrt{ }$ \\
\hline 17 Podocarpaceae & Podocarpus neriifolius & $\sqrt{ }$ & $\sqrt{ }$ & $\sqrt{ }$ & $\sqrt{ }$ & $\sqrt{ }$ & $\sqrt{ }$ & $\sqrt{ }$ \\
\hline 18 Sapotaceae & Madlutuca betis & & $\sqrt{ }$ & & & $\sqrt{ }$ & & \\
\hline 19 & Madlutuca boerlageana & & $\sqrt{ }$ & & & & & \\
\hline
\end{tabular}


Table 3: Timber tree species possibly in international trade and in need of taxonomic revision

Family

1 Araucariaceae

2

3

4 Ebenaceae

5

6 Leguminosae 7
Species

Agathis bomeensis

Agathis dammara

Agathis endertii

Diospyros blancoi

Diospyros ferrea

Dalbergia bariensis

Dalbergia cambodiana

[also listed in table 1]
Range States\#

KH ID LA MY PH TH VN Other $\sqrt{ }$ $\sqrt{ } \quad \sqrt{ }$

G G

$\underline{G} \underline{G} \quad \underline{G} \quad \underline{G} \quad \underline{G}$

G $\quad \underline{G}$

G $\underline{G}$

$\underline{\mathrm{G}}$

\# G indicates the species is from a genus identified as a priority

\section{Other Species}

The remaining 50 species, allocated to categories 4-6, are listed in tables 4-6.

Table 4: Timber tree species in international trade, not considered to be of conservation concern

\begin{tabular}{|c|c|c|c|c|c|c|c|c|}
\hline Family & Species & $\mathrm{KH}$ & ID & $\begin{array}{l}\text { ans } \\
\text { MY }\end{array}$ & $\begin{array}{l}\text { St } \\
\mathrm{PH}\end{array}$ & $\begin{array}{l}\text { tes } \\
\Gamma H\end{array}$ & VN & ther \\
\hline 1 Anacardiaceae & Mangifera decandra & & $\sqrt{ }$ & $\sqrt{ }$ & & & & $\sqrt{ }$ \\
\hline 2 Apocynaceae & Dyera costulata & & $\sqrt{ }$ & $\sqrt{ }$ & & $\sqrt{ }$ & & $\sqrt{ }$ \\
\hline 3 Araucariaceae & Arancaria cumninglamii & & $\sqrt{ }$ & $\sqrt{ }$ & & & & \\
\hline 4 Bombacaceae & Durio dulcis & & $\sqrt{ }$ & $\sqrt{ }$ & & & & \\
\hline 5 & Durio kutejensis & & $\sqrt{ }$ & $\sqrt{ }$ & & & & $\sqrt{ }$ \\
\hline 6 Burseraceae & Santiria laevigata & & $\sqrt{ }$ & & & $\sqrt{ }$ & & $\sqrt{ }$ \\
\hline 7 & Triomma malaccensis & & $\sqrt{ }$ & $\sqrt{ }$ & & & & $\sqrt{ }$ \\
\hline 8 Datiscaceae & Octomeles sumatrama & & $\sqrt{ }$ & $\sqrt{ }$ & & & & \\
\hline 9 Lauraceae & Delinasia caesin & & $\sqrt{ }$ & $\sqrt{ }$ & & & $\sqrt{ }$ & $\sqrt{ }$ \\
\hline 10 & Deluansia cumeata & $\sqrt{ }$ & $\sqrt{ }$ & $\sqrt{ }$ & & $\sqrt{ }$ & $\sqrt{ }$ & \\
\hline 11 Leguminosae & Sindorn beccariana & & $\sqrt{ }$ & $\sqrt{ }$ & & & & $\sqrt{ }$ \\
\hline 12 Olacaceae & Oclanostacliys amentacea & & $\sqrt{ }$ & $\sqrt{ }$ & & & & $\sqrt{ }$ \\
\hline 13 Sapotaceae & $\begin{array}{l}\text { Palaquinun } \\
\text { imıressinervinm }\end{array}$ & & & $\sqrt{ }$ & & $\sqrt{ }$ & & \\
\hline 14 & Palaquinm maingayi & & & $\sqrt{ }$ & & $\sqrt{ }$ & & \\
\hline
\end{tabular}




\begin{tabular}{|c|c|c|c|c|c|c|c|c|}
\hline Family & Species & \multirow{2}{*}{\multicolumn{7}{|c|}{$\begin{array}{l}\text { Range States } \\
\text { KH ID LA MY PH TH VN Other } \\
\sqrt{ }\end{array}$}} \\
\hline 15 Sterculiaceae & Pterocymbium beccarii & & & & & & & \\
\hline 16 Verbenaceae & Gmelina arborea & $\sqrt{ }$ & $\sqrt{ }$ & $\sqrt{ }$ & $\sqrt{ }$ & $\sqrt{ }$ & $\sqrt{ }$ & $\sqrt{ }$ \\
\hline 17 & Tectona grandis & $\checkmark \quad \sqrt{ }$ & $\sqrt{ }$ & $\sqrt{ }$ & & $\sqrt{ }$ & $\sqrt{ }$ & $\sqrt{ }$ \\
\hline
\end{tabular}

Table 5: Timber tree of conservation concern not thought to be in international trade

\begin{tabular}{|c|c|c|c|c|c|c|c|c|}
\hline Family & Species & KH ID & $\begin{array}{r}\text { Ran } \\
\text { LA M }\end{array}$ & $\begin{array}{l}\text { nge } S \\
\text { IY PH }\end{array}$ & $\begin{array}{l}\text { Stat } \\
\text { H T }\end{array}$ & $\begin{array}{l}\text { tes } \\
\mathrm{HI}\end{array}$ & & Other \\
\hline 1 Burseraceae & Canarimm luzonicum & & & $\sqrt{ }$ & $\sqrt{ }$ & & & \\
\hline 2 Cephalotaxaceae & Cephalotaxus oliveri & & $\sqrt{ }$ & & & & $\sqrt{ }$ & \\
\hline 3 Fagaceae & Fagus longipetiolata & & & & & & $\sqrt{ }$ & \\
\hline 4 Lauraceae & Cinnamommm porrectum & $\sqrt{ }$ & & & & & $\sqrt{ }$ & $\sqrt{ }$ \\
\hline 5 Leguminosae & Afzelia rhomboidea & $\sqrt{ }$ & $\sqrt{ }$ & $\sqrt{ }$ & $\sqrt{ }$ & & & \\
\hline 6 & Kalappia celebica & $\sqrt{ }$ & & & & & & \\
\hline 7 & Pericopsis mooniana & $\sqrt{ }$ & $\sqrt{ }$ & $\sqrt{ }$ & j & & & \\
\hline 8 & $\begin{array}{l}\text { Wallaceodendron } \\
\text { celebicum }\end{array}$ & $\checkmark$ & & $\sqrt{ }$ & j & & & \\
\hline 9 Meliaceae & Aglaia perviridis & & $\sqrt{ }$ & $\sqrt{ }$ & & $\sqrt{ }$ & $\sqrt{ }$ & \\
\hline 10 & Aglain silzestris & $\sqrt{ }$ & $\sqrt{ }$ & $\sqrt{ }$ & 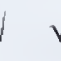 & $\sqrt{ }$ & $\sqrt{ }$ & \\
\hline 11 & Toona calantas & $\sqrt{ }$ & $\sqrt{ }$ & $\checkmark$ & 1 & & & \\
\hline 12 Olacaceae & Strombosia javanica & $\sqrt{ }$ & $\sqrt{ }$ & $\sqrt{ }$ & & $\sqrt{ }$ & & $\sqrt{ }$ \\
\hline 13 Rutaceae & Merrillia caloxylon & $\sqrt{ }$ & $\sqrt{ }$ & v & & $\sqrt{ }$ & & $\checkmark$ \\
\hline 14 Sapotaceae & Palaquium bataanense & & & $\sqrt{ }$ & 1 & & & \\
\hline 15 Sterculiaceae & Scaplitum longiflonum & $\sqrt{ }$ & $\sqrt{ }$ & v & & & & \\
\hline 16 Verbenaceae & Tectona philippinensis & & & $\sqrt{ }$ & 1 & & & \\
\hline 17 & Vitex parviflora & $\checkmark$ & & $\sqrt{ }$ & 1 & & & \\
\hline
\end{tabular}


Table 6: Timber tree species not thought to be in international trade or to be of conservation concern

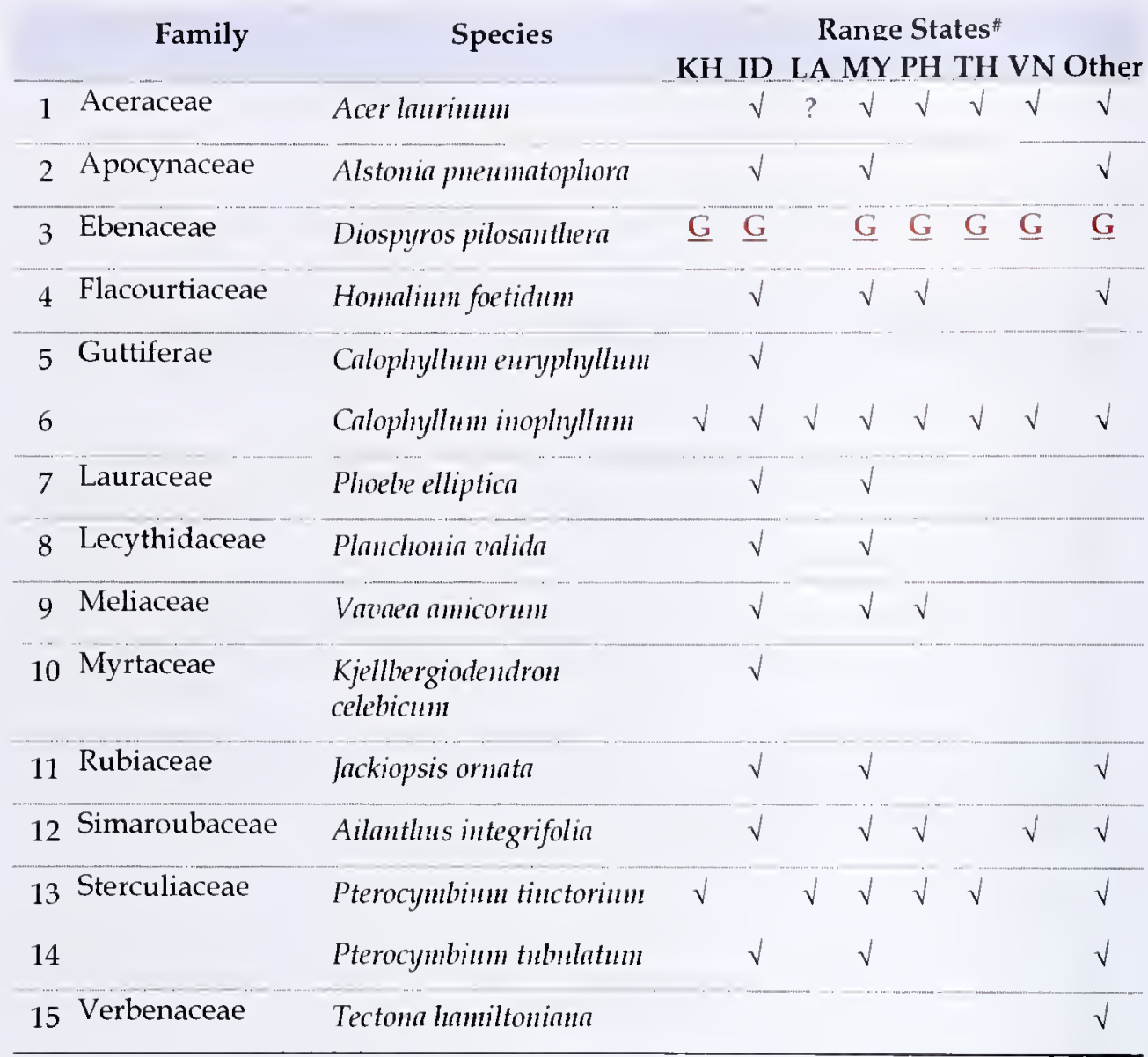

${ }^{*} \underline{G}$ indicates the species is from a genus identified as a priority 


\section{CONCLUSIONS}

1. The workshop report should be sent as a priority to:

- Range state national authorities, for consideration on actions concerning the species for which they are a range state.

$>$ the CITES Plants Committee, for consideration concerning species in international trade

$>1 \mathrm{UCN} \quad(\operatorname{Red} \quad$ List programme), for consideration concerning species identified as threatened.

2. Species in four genera were identified as being in need of taxonomic revision:

$>$ Agathis (kauri/dammar) Araucariaceae (A taxonomic revision of Agathis in Indonesia was not considered necessary by the Indonesian participants).

$>$ Cynonuetra

(kekatong) Leguminosae

$>$ Dalbergia (rosewoods) Leguminosae

$>$ Diospyros (ebonies) Ebenaceae

Two of these genera, Dalbergia and Diospyros were noted as being of particular importance.
Given the taxonomic confusion within these genera and the identification of Dalbergia species as priority species in both the Central American and the South East Asian workshops, it was suggested that the two genera should be priorities for taxonomic and subsequent trade review. UNEP-WCMC will forward the document to the CITES Nomenclature Committee, Royal Botanic Garden Kew and other taxonomic organisations.

3. Stefan Verbunt, on behalf of the government of the Netherlands, noted in his concluding speech, that the responsibility to prepare proposals to include any species in CITES Appendices I or II rested with range states, but that the Netherlands would be willing to provide support to range states in the preparation of such proposals.

4. Participants noted that it would be important to run a follow-up workshop in the region, to assess action in relation to the species listed in tables $1,1 \mathrm{~A}, 2$ and 3 and to identify relevant actions needed as well as to consider additional species, including those listed in the table $1 \mathrm{~A}$. 
Regional representatives

\author{
CAMBODIA \\ Mr. Neang Thy \\ Officer of National Park and Wildlife \\ Sanctuary Office \\ Department of Nature Conservation \\ and Protection \\ Ministry of Environment \\ CAMBODIA \\ T: +85516 671771 \\ E: neangthy@yahoo.com
}

\section{INDONESIA}

Dr. Eko Baroto Walujo

Head of Botany Division

Keeper of Herbarium Bogoriense

Research Centre for Biology

Indonesian Institute of Sciences

Herbarium Bogoriense

Bidang Botani, Puslit Biologi-LIPI

Cibinong Science Centre (CSC) - LIPI

Jalan Raya Jakarta-Bogor Km. 46

Cibinong 16911

INDONESIA

$\mathrm{T}:+62251 / 322035$

F: $+62251 / 336538$

E: herbogor@indo.net.id

Dr. Tukirin Partomihardjo

Research Centre for Biology

Indonesian Institute of Sciences

Herbarium Bogoriense

Bidang Botani, Puslit Biologi-LIPI

Cibinong Science Centre (CSC) - LIPI

Jalan Raya Jakarta-Bogor Km. 46

Cibinong 16911

CITES Regional Plant Committee (Asia)

INDONESIA

$\mathrm{T}:+62218765066$

F: + 62218765063

Dr. Teguh Triono

Research Centre for Biology

Indonesian lnstitute of Sciences
Herbarium Bogoriense

Bidang Botani, Puslit Biologi-LIPI

Cibinong Science Centre (CSC) - LPl

Jalan Raya Jakarta-Bogor Km. 46

Cibinong 16911

INDONESIA

E: ttriono@cbn.net.id

Tajudin Edy Komar

Department of Forestry

Forestry Research and Development

Agency

Forest and Nature Conservation

Research and Development Centre

Jl. Gunung Batu No. 5

Bogor

INDONESIA

T: +251 633234, 7520067

F: +251638111

E: raminppd87@vahoo.com

Mrs. Titiek Setyawati

Department of Forestry

Forestry Research and Development

Agency

Forest and Nature Conservation

Research and Development Centre

Jl. Gunung Batu No. 5

Bogor

INDONESIA

T: +251633234

F: +251638111

E: titiek29@yahoo.com

\section{LAO PDR}

Dr. Bounthong Bouahom

Department of Forestry

Vientiane

Forest Resources Conservation

Division

Head of CITES unit

Lao PDR 
Tel: $+856-21215000,+856-202401099$

Fax: +856-217161

E: phobounthanh@yahoo.com

Saysamone Phothisat

Department of Forestry

Vientiane

Lao PDR

Tel: +856-21 215000, 205336888

Fax: $+856-21222552$

E: saiphothisat@yahoo.com

\section{MALAYSIA}

Dr. Lillian Chua

Forest Research Institute Malaysia (FRIM)

52109 Kepong,

Selangor Darul Ehsan

MALA YSIA

T: +60 (3) 62797223

F: +60 (3) 62804625

E: Lilian@frim.gor.my

Julaihi Abdullah

Research Manager

Applied Forest Science \& Industry

Development (AFSID)

Sarawak Forestry Corporation

Forest Research Centre

Jalan Datuk Amar Kalong Ningkan 93250 Kuching, Sarawak

MALAYSIA

T: +6082614826

F: +6082617953

Toll Free Line: 1-800-88-ALAM

E: julaihilai@sarawakforestry.com

Mr. John Sugau

Jabatan Perhutanan Sabah

Senior Research Officer

Herbarium Forest Research Centre

Sabah Forestry Department

P.O.Box 1407, Sepilok

90715 Sandakan

Sabah

MALAYSIA

E: Iohn.Sugau@sabah.gov.my

Dr. Saw Leng Guan

Director
Tropical Forest Biodiversity Centre Forest Research Institute Malaysia 52109 Kepong, Selangor

\section{MALAYSIA}

T: +603-62797218

F: $+603-62731041$

E: sawlg@frim.gov.my

Dr. Lee Hua Seng

Head of Secretariat

Sarawak Timber Association

11 Floor, Wisma STA

Jalan Datuk Abang Abdul Rahim, 93450 Kuching,

Sarawak

MALAYSIA

$\mathrm{T}:+(6082) 332222$

F: $+(6082) 487888,487999$

E: hslee@sta.org.my

Aimi Lee Abdullah (Mrs)

Malaysian Timber Council

$18^{\text {th }}$ Floor, Menara PGRM,

No. 8, Jalan Pudu Ulu

Cheras

56100 Kuala Lumpur

MALAYSIA

Dr. David Chin

18th Floor, Menara PGRM,

No. 8, Jalan Pudu Ulu

Cheras

56100 Kuala Lumpur

MALAYSIA

\section{PHILIPPINES}

Dr. Domingo Madulid

Curator

Philippines National Museum

National Museum

P.O. Box 2659

Manila

THE PHILIPPINES

E: dmadulid@info.com.ph

Dr. Antonio Manila, Chief, Wildlife

Division

Protected Areas and Wildlife Bureau

Diliman,

Department of Environment and 
Natural Resources.

Quezon City

THE PHILIPPINES

T: + 6329246031 loc 222

Dr. Honorato Palis

Ecosystems Research and

Development Bureau College,

Laguna, 4031

THE PHILIPPINES

T: + $63495362269 / 5362229$

F: + 63495362850

E: hgpalis@lgn.pworld.net.ph

E: palishg@yahoo.com

\section{THAILAND}

Dr. Kongkanda Chayamarit

Director

Forest Herbarium (BKF)National Park

Wildlife and Plant Conservation

Department

61 Phahonyothin Rd., Chatuchak,

Bangkok 10900

THAILAND F: + 6625614824

T: + 662 5614292-3 Ext. 814

E: kchayama@mozart.inet.co.th
Dr. Kitichate Sridith

Associate Professor

Faculty of Science, Prince of Songkla

University

Hatyai, Songkhla 90112

THAILAND

E: Kitichate.S@psu.ac.th.

\section{VIETNAM}

Tran Ngoc Hai

Forest Plant Department

The Vietnam Forestry University

Xuan Mai - Ha Tay

VIETNAM

Tel: 008434840628

Cell phone: 0084912206245

Email: haicrungfu@yahoo.com

Mr. Hoang Van Sam

Forest Plant Department

The Vietnam Forestry University

Xuan Mai - Ha Tay

VIETNAM

E: vanhoang@nhn.leidenuniv.nl

Others

\section{AUSTRALIA}

Greg Leach

Biodiversity Conservation

Department of Natural Resources,

Environment \& the Arts

PO Box 496

Palmeston NT 0831

AUSTRALIA

T: +61889994520

M: +61 401118454

F: +61 89994527

E: greg.leach@nt.gov.au

\section{THE NETHERLANDS}

Jan de Koning

CITES Scientific Authority of the

Netherlands

Nationaal Herbarium Leiden

P.O.Box 9514

2300 RA Leiden

THE NETHERLANDS
T: +31715273559 (work)

T: +31 629571095

E: DeKoning@nhn.leidenuniv.nl

Chris Schürmann

CITES Scientific Authority of the

Netherlands

National Museum of Natural History

Naturalis

Postbus 9517 - 2300 RA Leiden

THE NETHERLANDS

$\mathrm{T}:+31715687591$;

F: +31715687666

E: c.l.schurmann@minlnv.nl

Stefan Verbunt

Ministry of Agriculture, Nature and

Food Quality

Department of Nature

P.O. Box 20401

2500 EK The Hague

THE NETHERLANDS 
$\mathrm{T}:+31-70-3784736$

F: $+31-70-3786146$

E: s.j.d.verbunt@minlnv.n]

\section{UK}

James Paver

British High Commission

Kuala Lumpur

MALAYSIA

$\mathrm{T}:+603$

F: +603

E: James.Paver@fco.gov.uk

\section{USA}

Pat Ford

Botanist

Division of Scientific Authority

U.S. Fish and Wildlife Service

4401 N. Fairfax Dr., Room 750

Arlington, VA. 22203

USA
T: 703-358-1708
F: 703-358-2276
E:Patricia_Ford@fws.gov

\section{FAO}

Masakazu Kashio

Forestry Officer

FAO

Bangkok

THAILAND

E: Masakazu.Kashio@fao.org

\section{ITTO}

Steve Johnson

International Tropical Timber

Organization (ITTO)

International Organizations Center -

5F

Pacifico-Yokohama

1-1-1 Minato-Mirai, Nishi-ku

Yokohama 220-0012

JAPAN

Tel. +81452231110

Fax +81452231111

E: johnson@itto.or.jp

E: manoel.sobral@itto.or.jp

http://www.itto.or.jp

\section{IUCN/SSC}

Bian Tan

IUCN/SSC Global Trees Specialist

Group

BGCI SE Asia Programme Coordinator c/o Registry, Singapore Botanic

Gardens

1 Cluny Road, Singapore 259569

SINGAPORE

T/F: +65 64674206

M: +65 98248627

E: bian.tan@bgci.org

TRAFFIC

Chen Hin Keong

TRAFFIC International

TRAFFIC Southeast Asia Regional

Office

Unit 9-3A, 3rd Floor, Jalan SS23/11, Taman SEA, 47400 Petaling Jaya,

Selangor

MALAYSIA

T: +60378803940

F: +60378820171

E: hkchen@pc.jaring.my

Noorainie Awang Anak

Project Officer SE Asia

TRAFFIC Southeast Asia Regional

Office

Unit 9-3A, 3rd Floor, Jalan SS23/11,

Taman SEA, 47400 Petaling Jaya,

Selangor

MALAYSIA

T: +60378803940

F: +60378820171

E: naatsea@po.jaring.my

\section{UNEP-WCMC}

Harriet Gillett

Senior Programme Officer

Species Programme

UNEP-WCMC

219 Huntingdon Rd,

Cambridge CB3 ODL

UK

T: +44 1223277314 ex 250

F: +441223277136

E: harriet.gillett@unep-wcmc.org 
Pablo Sinovas

Research Assistant

Species Programme

UNEP-WCMC

219 Huntingdon Rd,

Cambridge CB3 0DL

UK

T: +44 1223277314

F: +44 1223277136

E: pablo.sinovas@unep-wcmc.org

Soh Wuu Kuang

Consultant

c/o UNEP-WCMC

219 Huntingdon $\mathrm{Rd}$,

Cambridge CB3 0DL

UK
T: +353-(0)1-8961421

M: +3530872368150

E: wuukuang@gmail.com

WWF

Ivy Wong

WWF-Malaysia

49, Jalan SS23/15

Taman SEA

47400 Petaling Jaya

Selangor

MALAYSIA

T: +60378033772

F: +60378035157

E: IWong@wwf.org.my

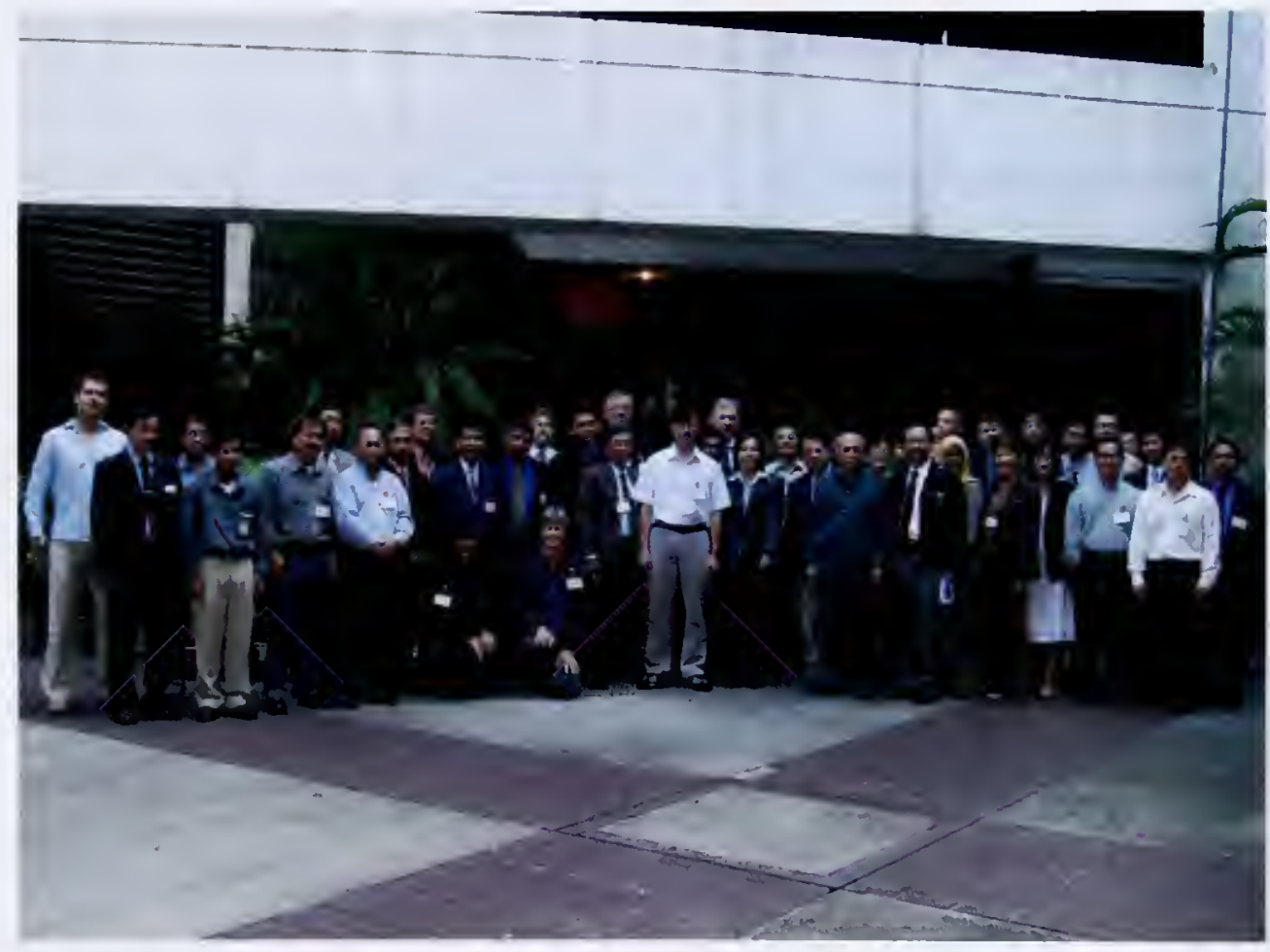

Workshop participants 
ANNEX 1

WORKSHOP SPECIES COMMENTS 


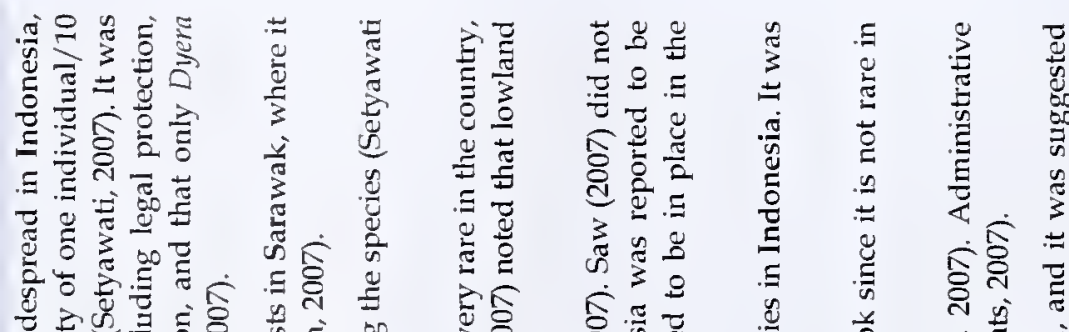

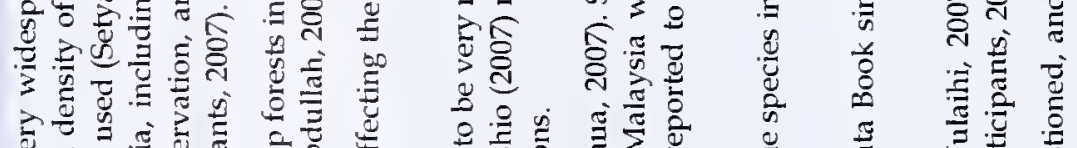

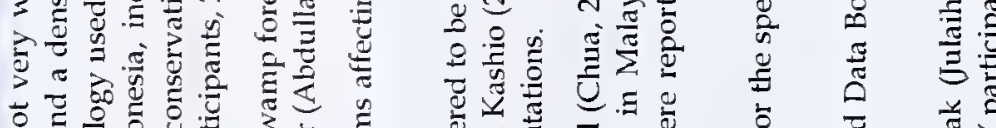

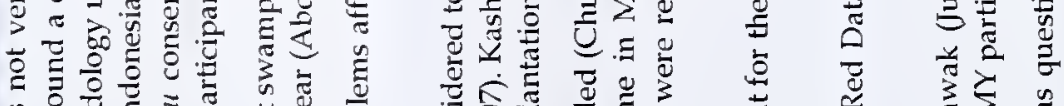

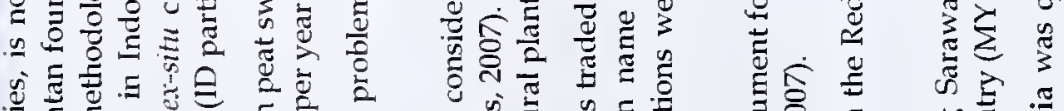

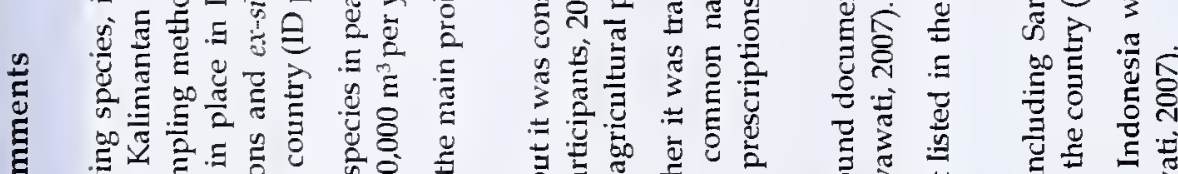

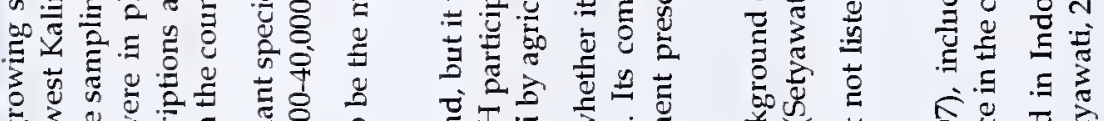

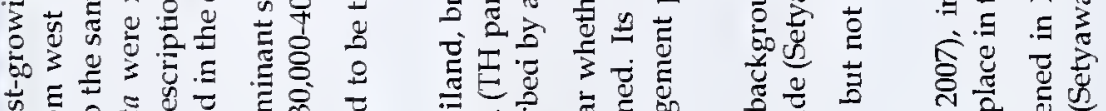

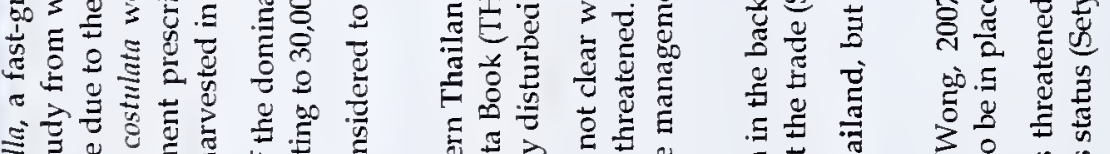

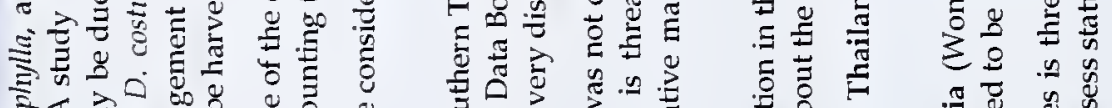

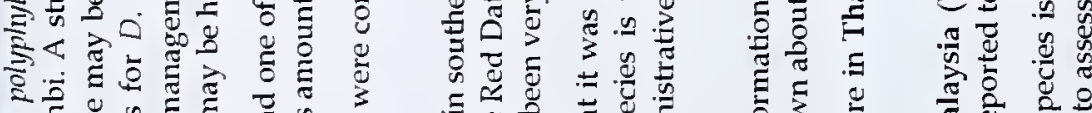

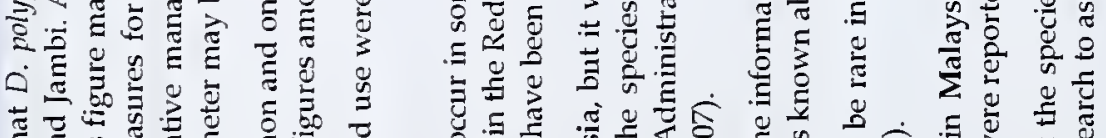

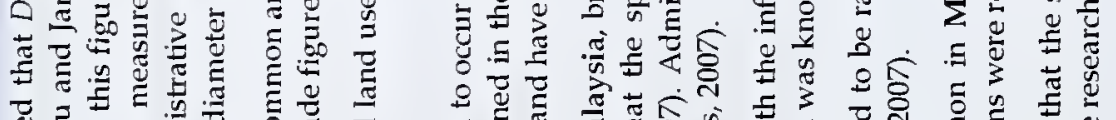

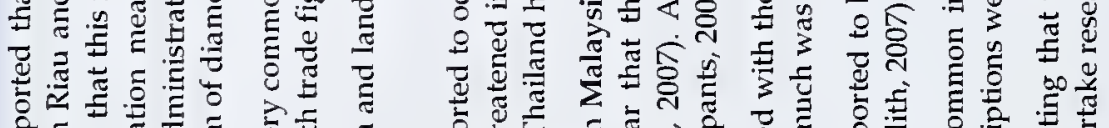

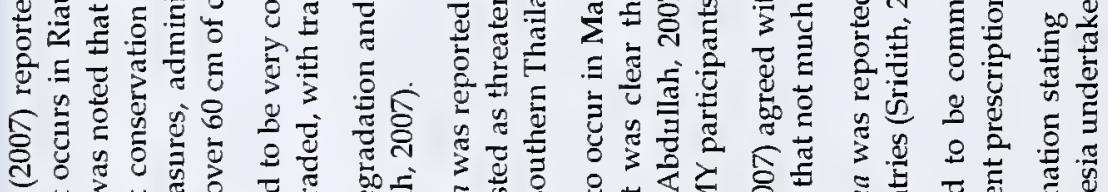

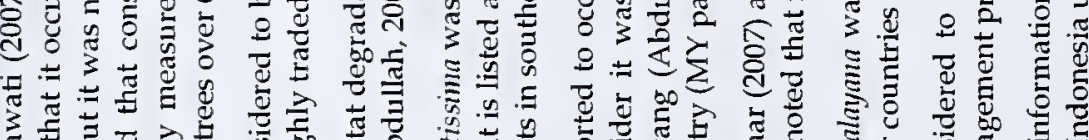

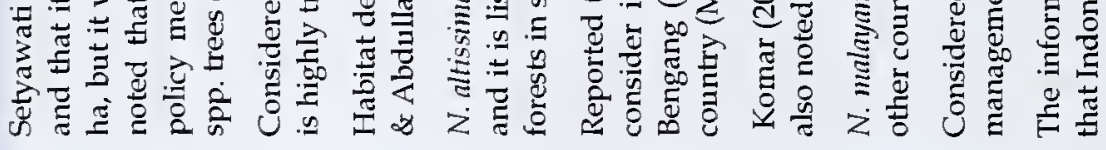
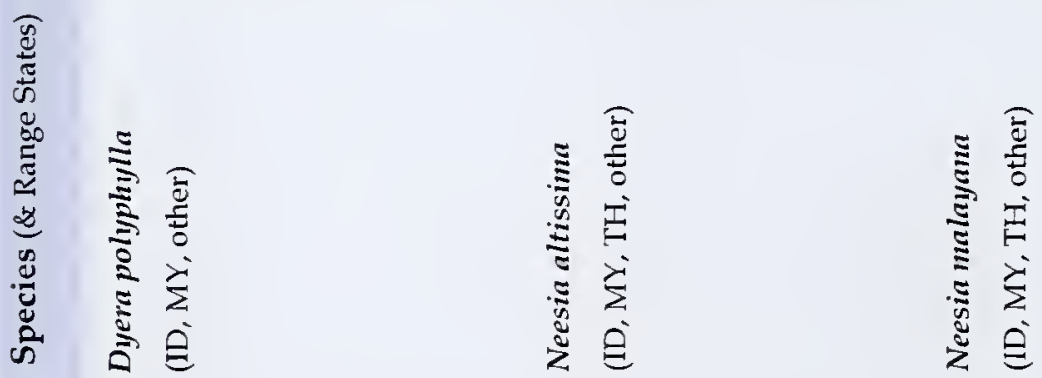

管

$\sum_{0}^{\substack{5 \\ 0}}$

N 


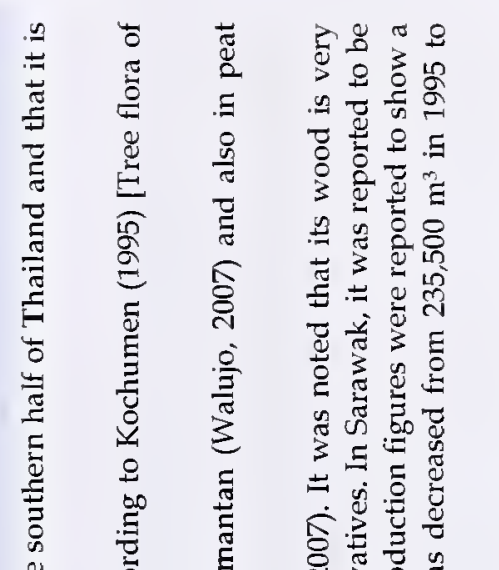

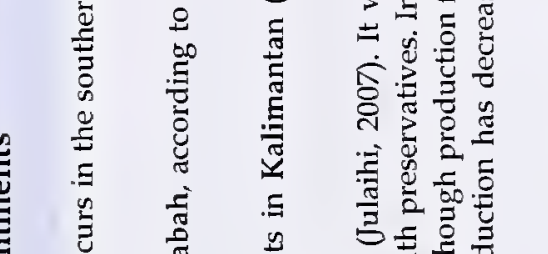

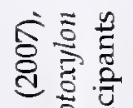

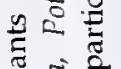

翼彭

(1)

旁

$\sum \sum \stackrel{0}{0}$

Ð

क.

范

造

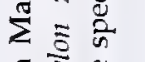

ำ

釆旁

空

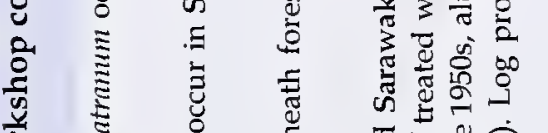

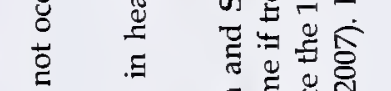

का मा च

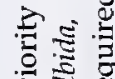

脑

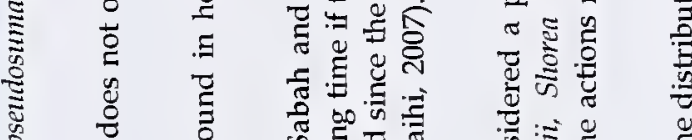

.

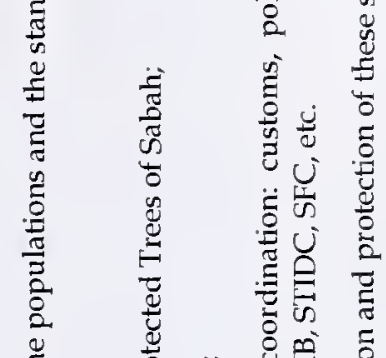

胥

ह

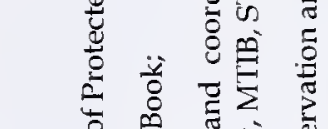

ह

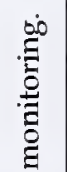

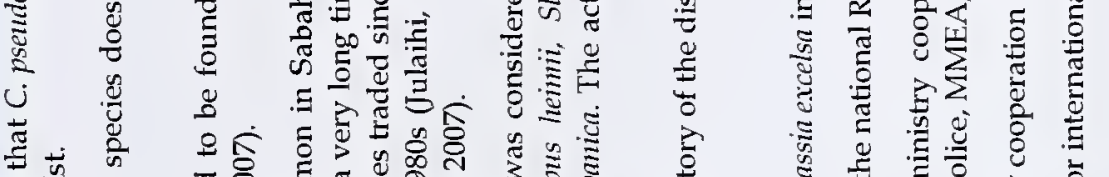

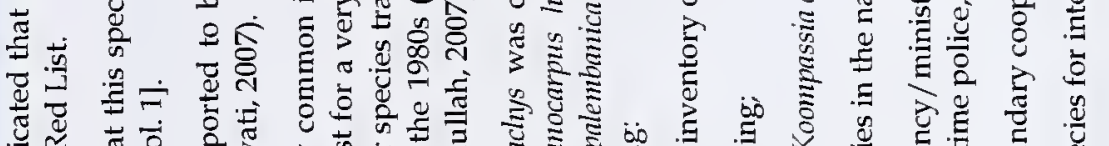

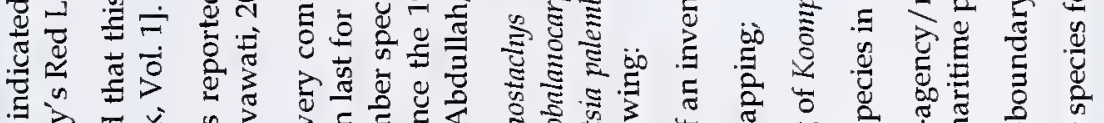

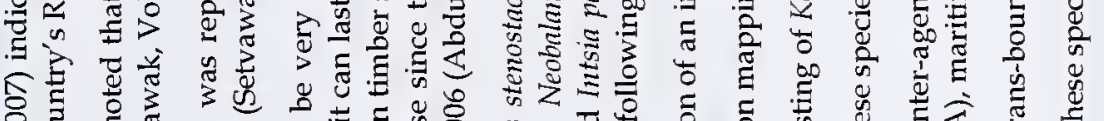

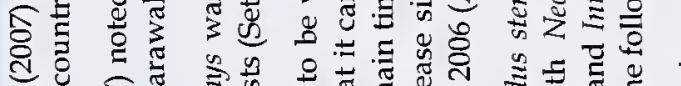

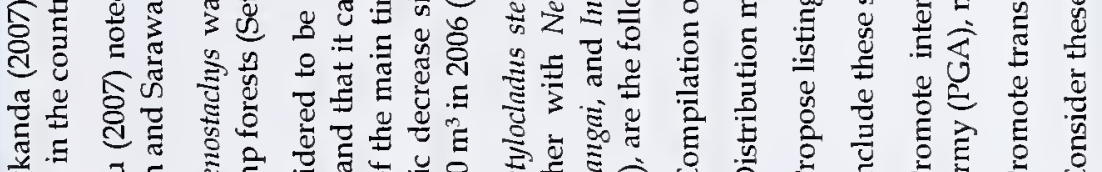

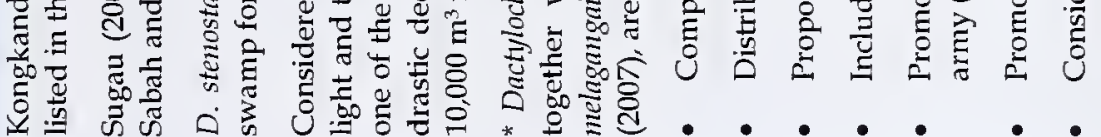

$\frac{\mathscr{8}}{\pi}$

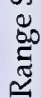

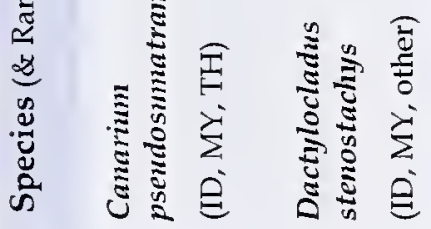

خ

in

o 


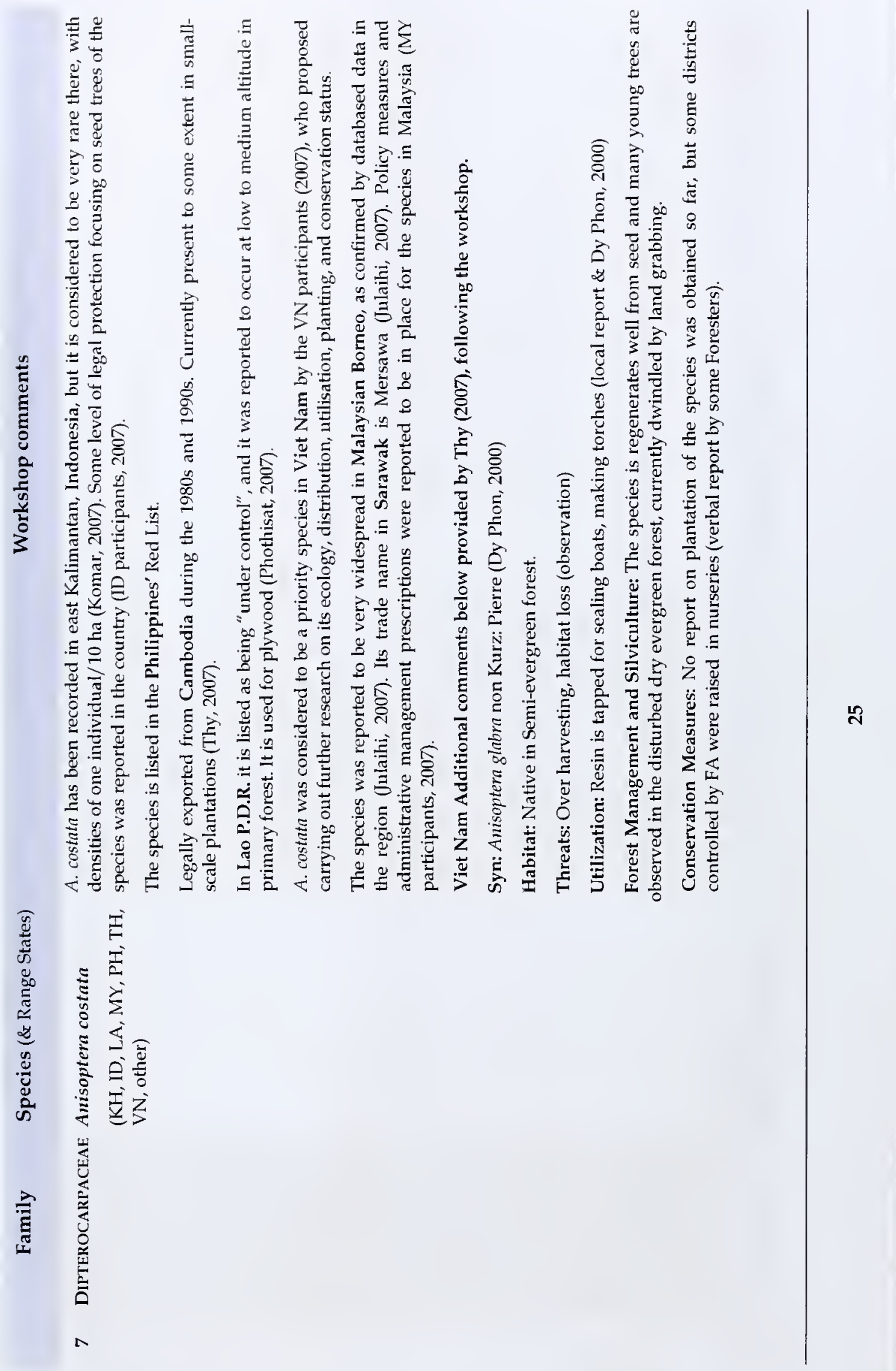




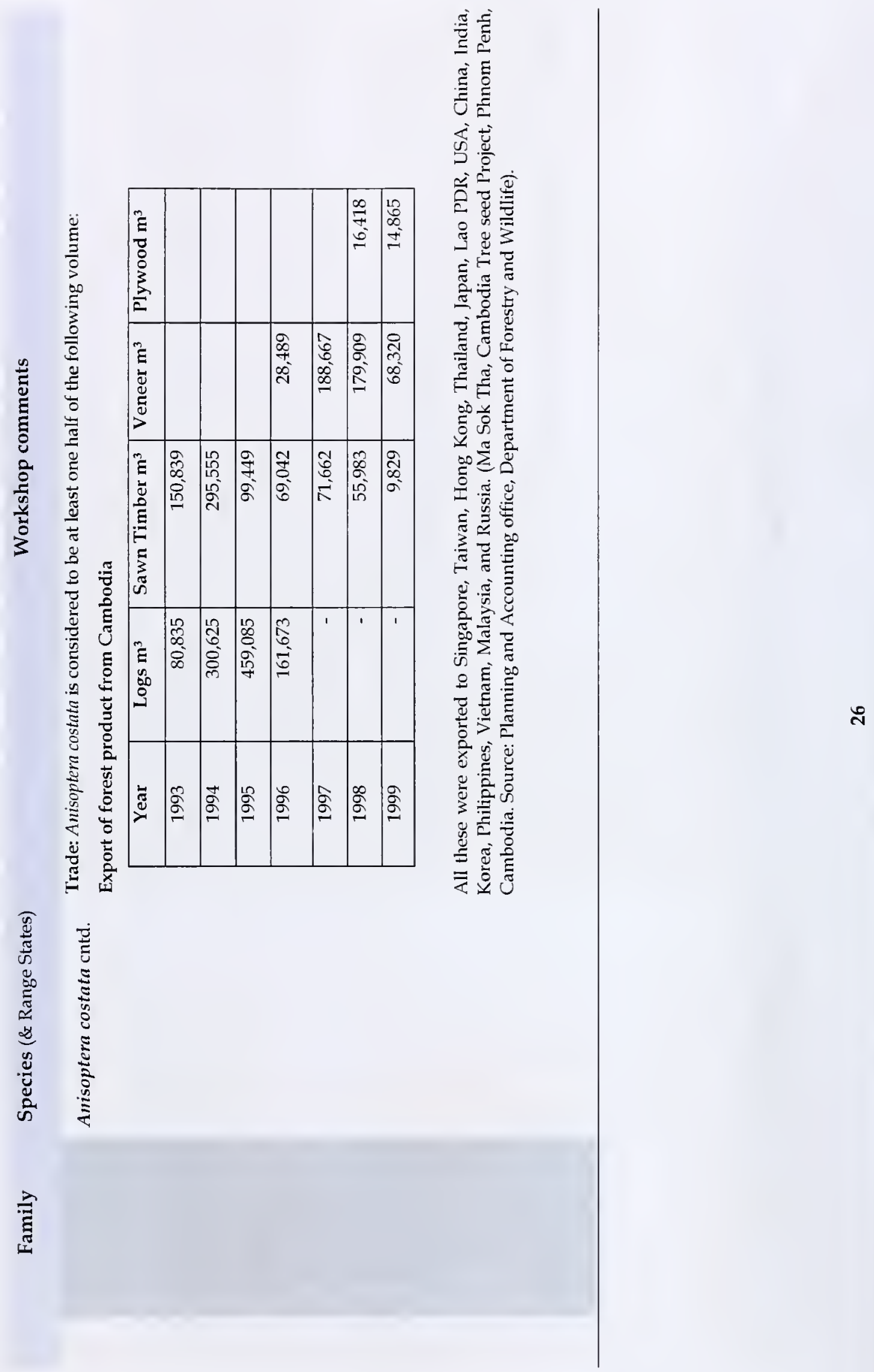




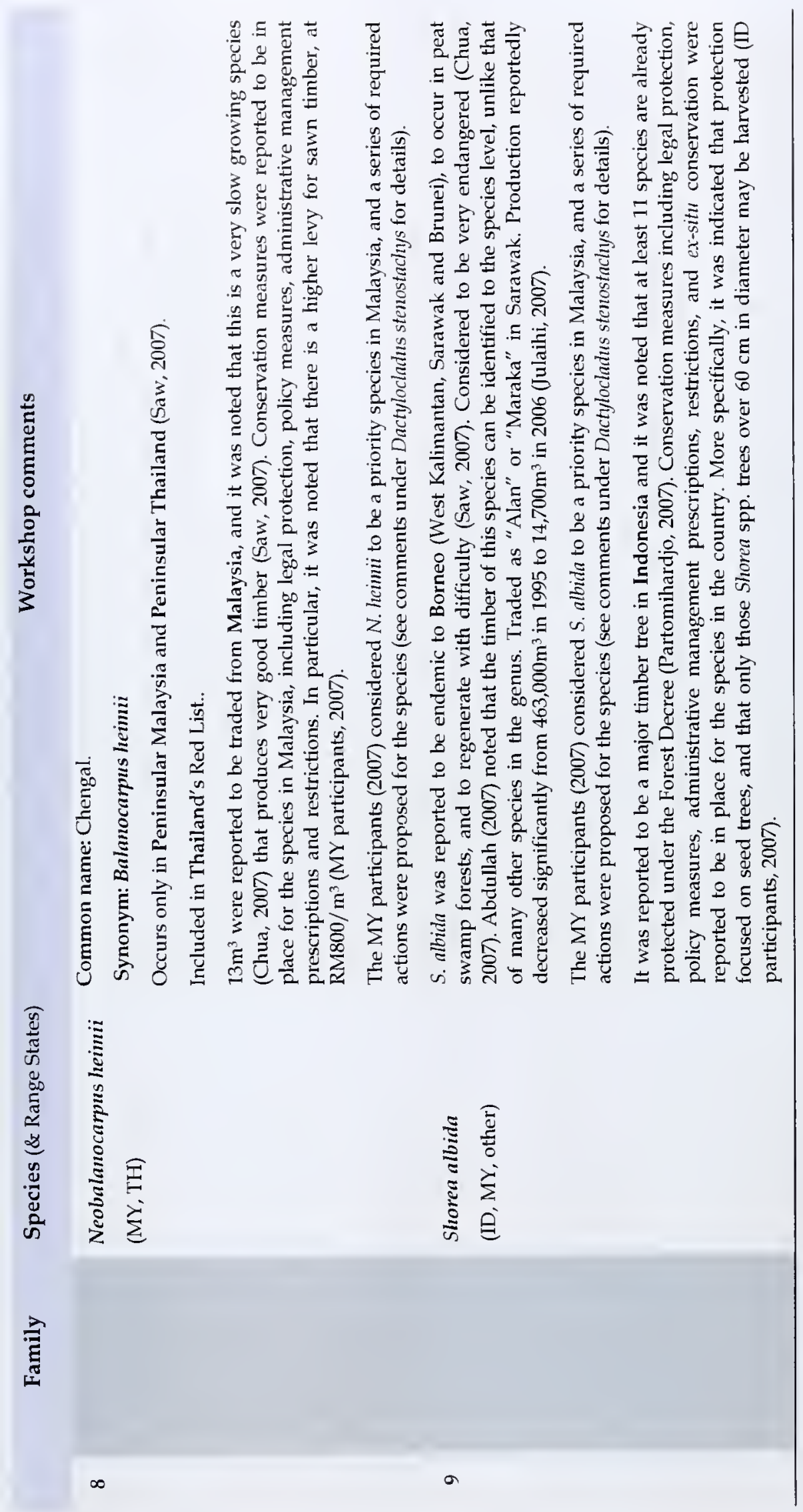

N 


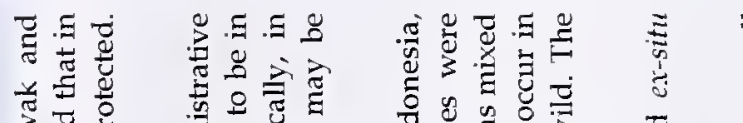

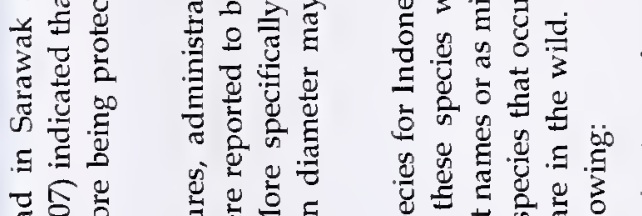

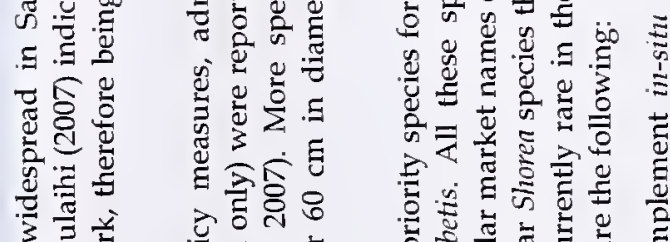

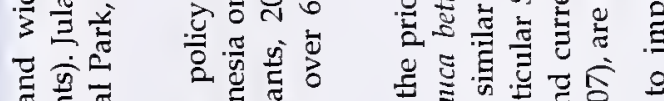

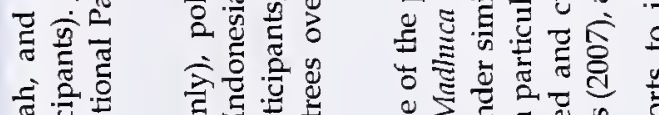

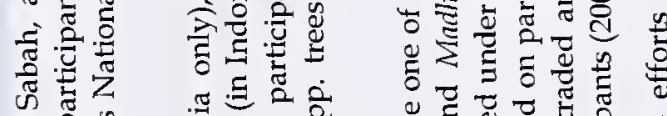

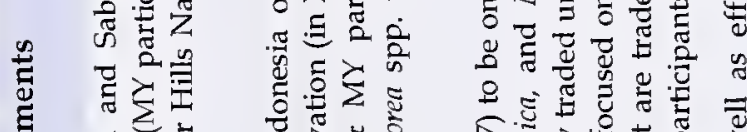

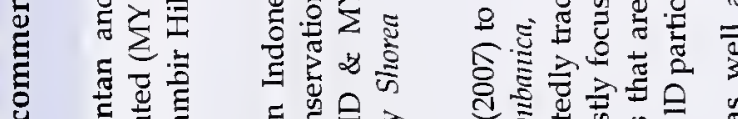

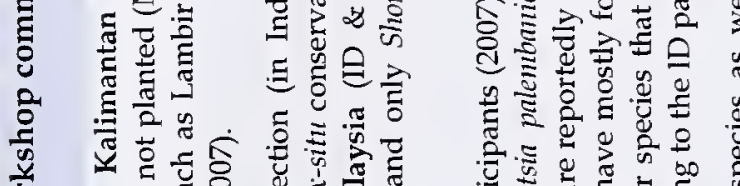

1)

5 放

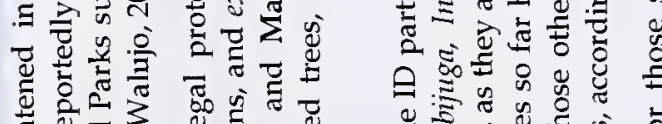

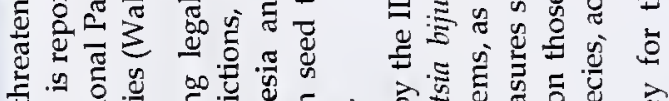

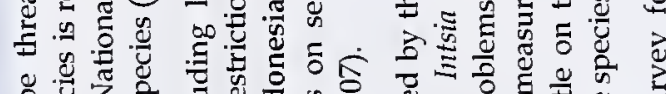

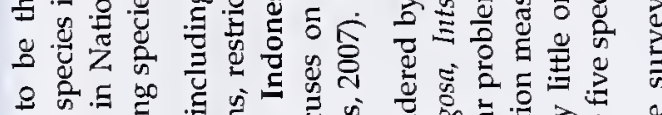

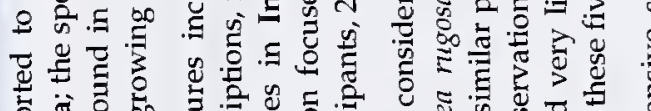

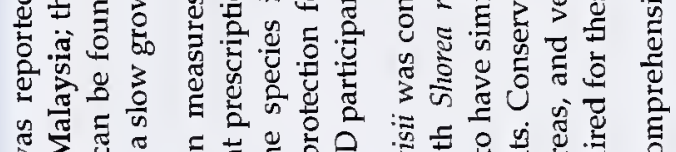

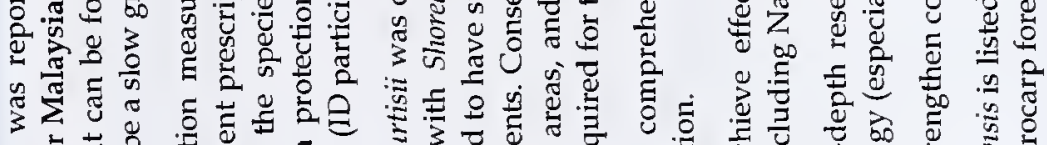

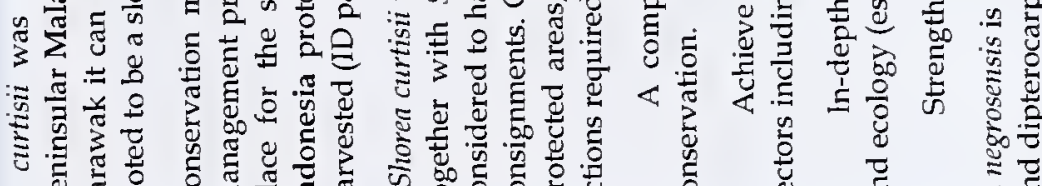

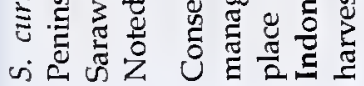

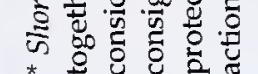

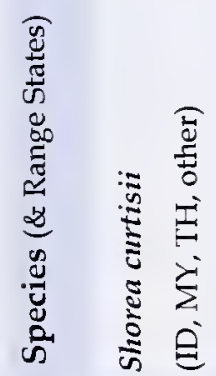

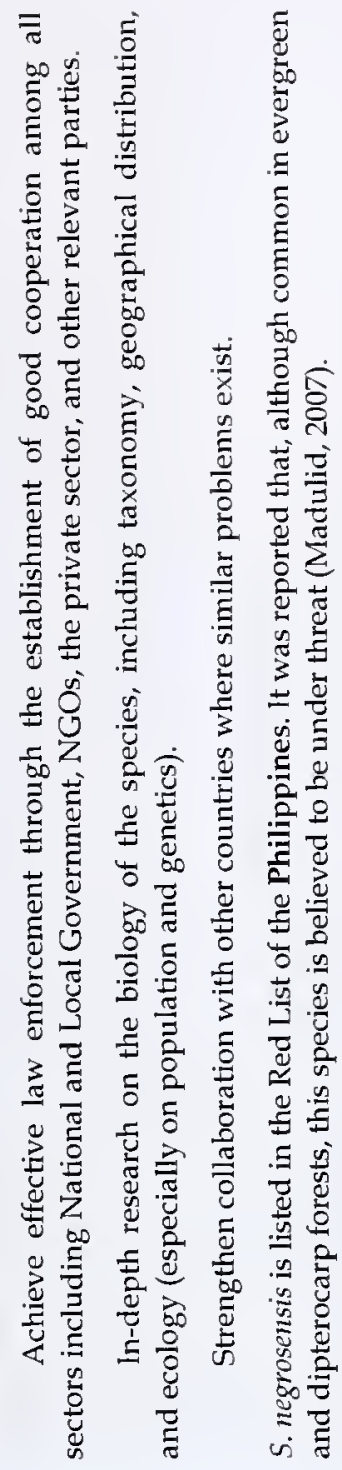




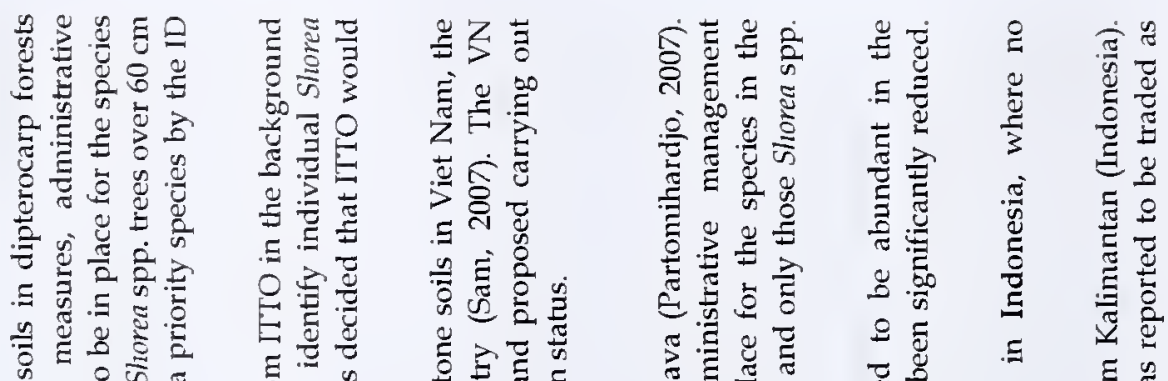

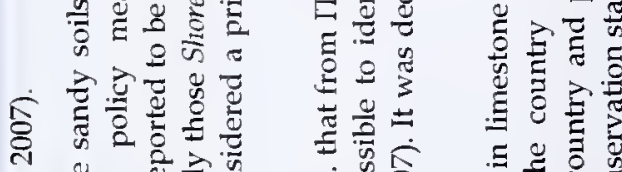

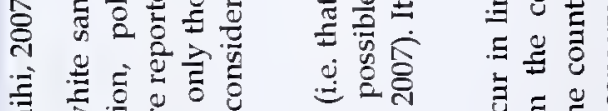

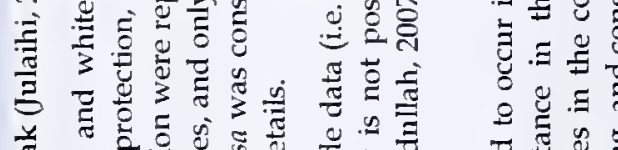

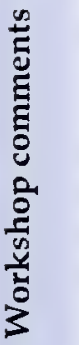

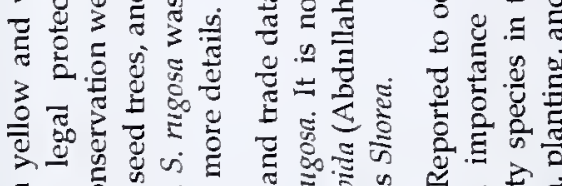

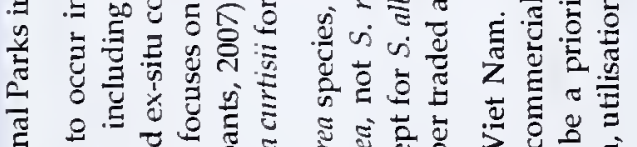

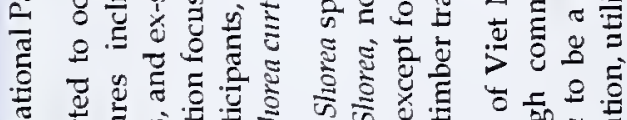

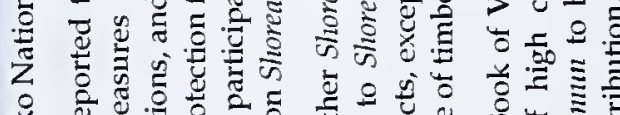

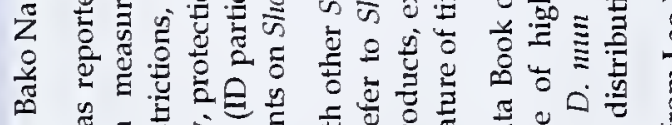

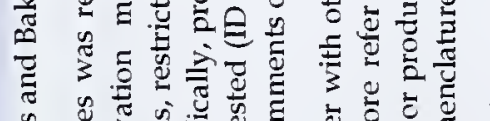

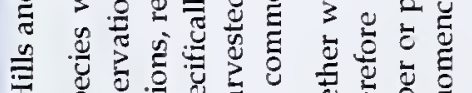

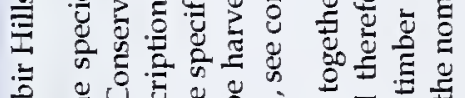
范

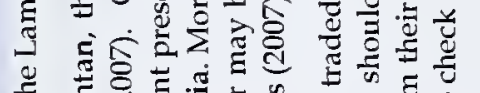

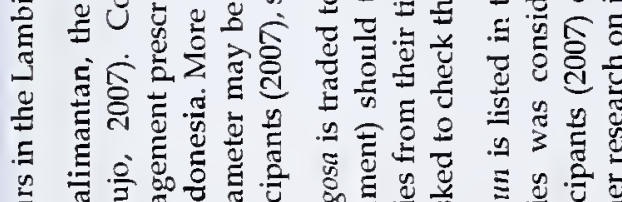

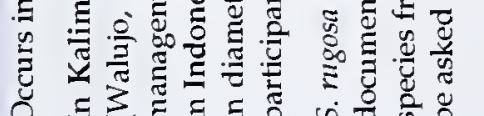
㱐

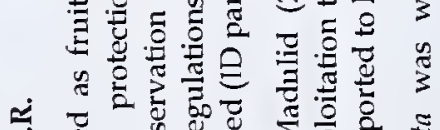

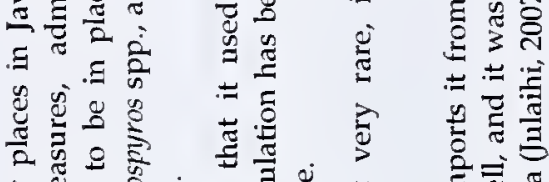

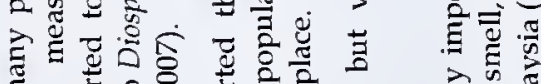

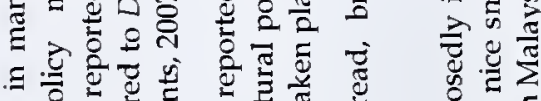

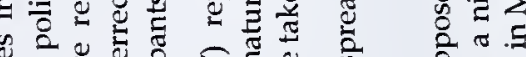

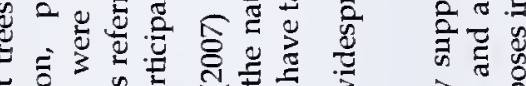

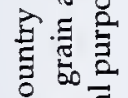
8 需

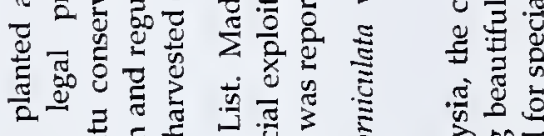

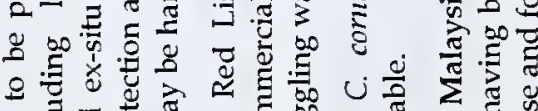

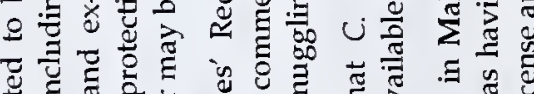

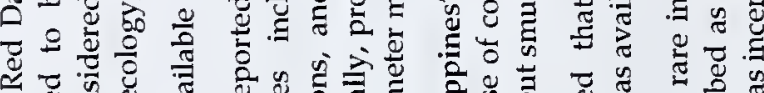

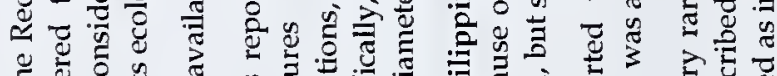

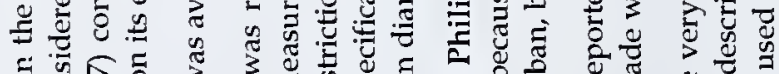
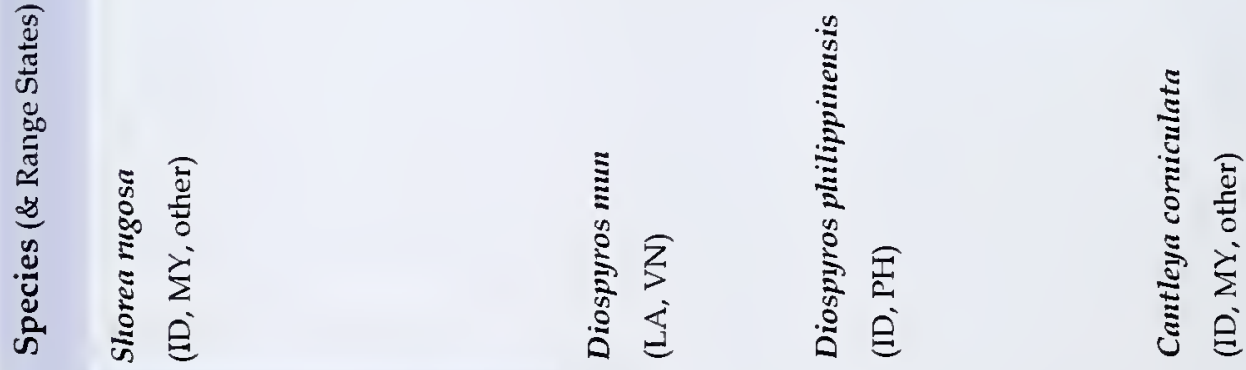

촐

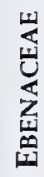



I

$m$

$\nexists$

$\stackrel{2}{2}$ 


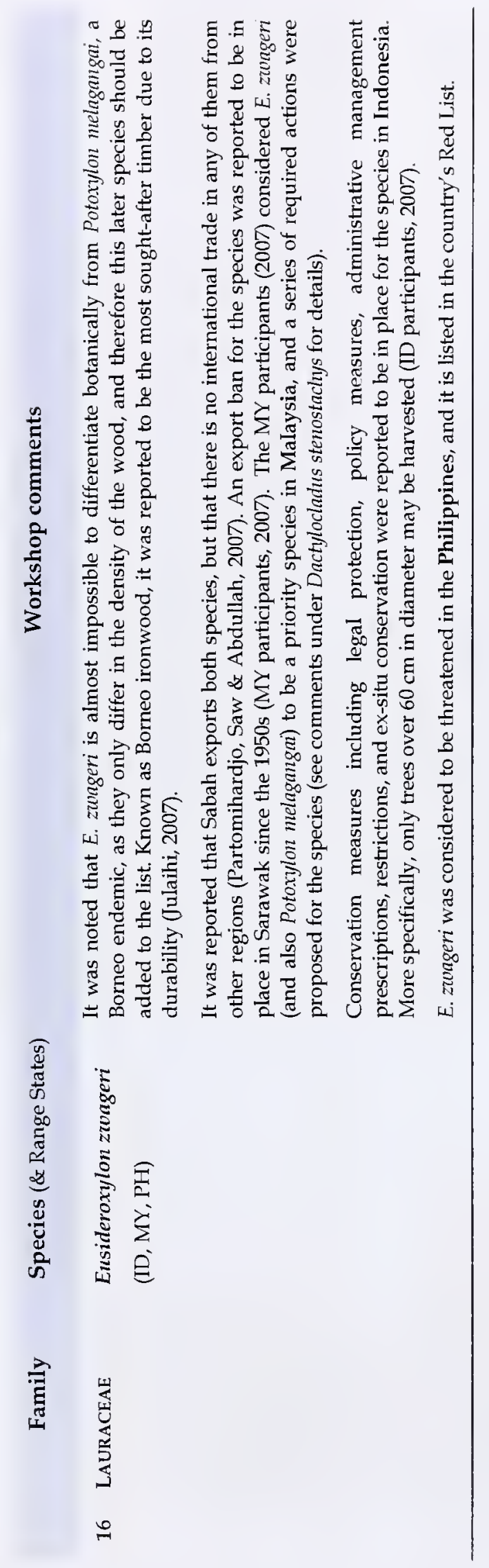

요 


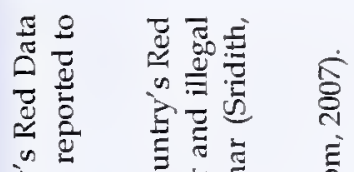

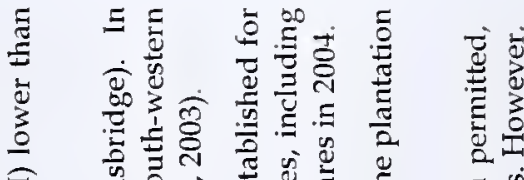

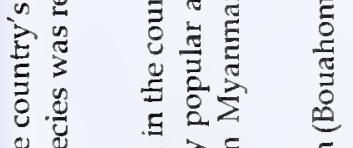

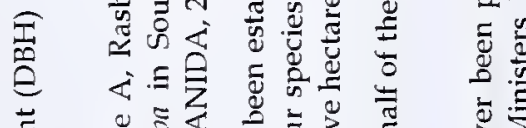

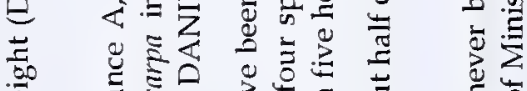

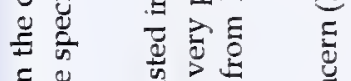

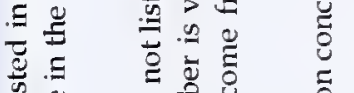

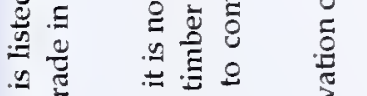

绕

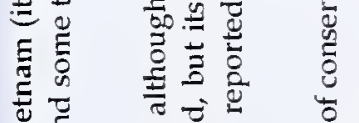

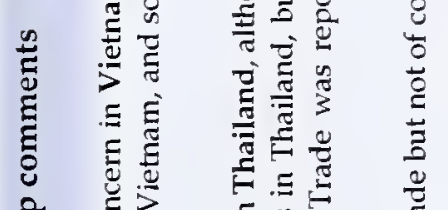

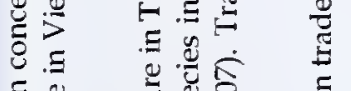

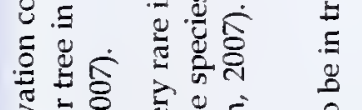

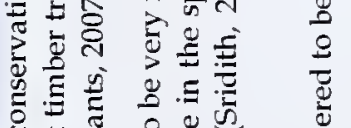

5)

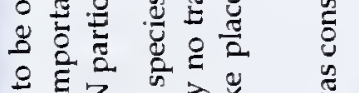

을

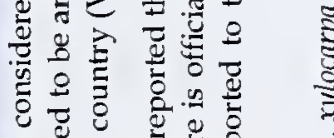

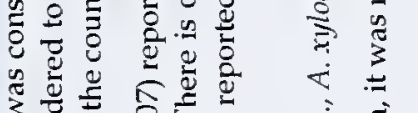

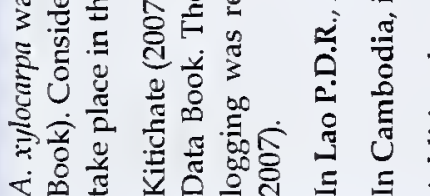

હ $\frac{0}{8}$

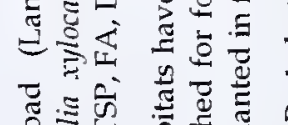

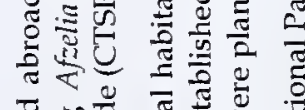

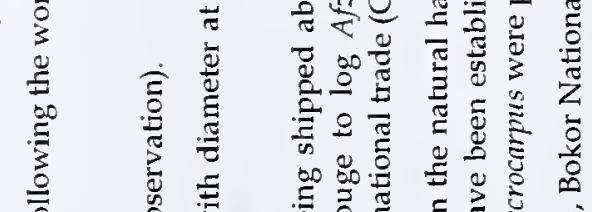

岁

这

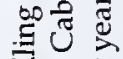

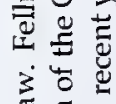

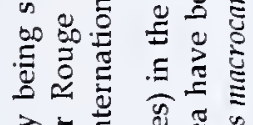

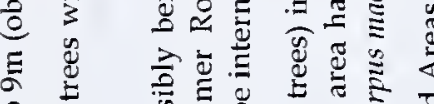

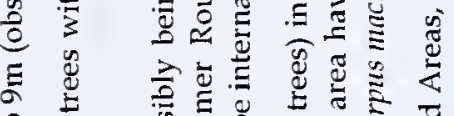

$\simeq \Xi$

글

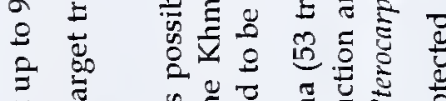

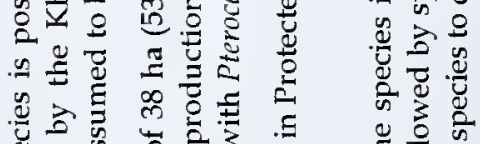

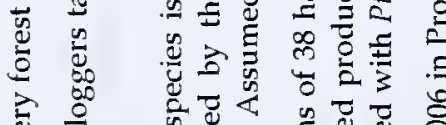

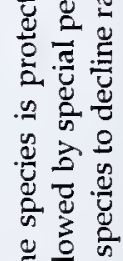

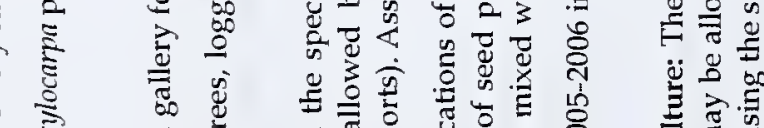

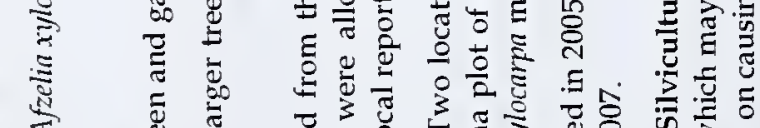

To

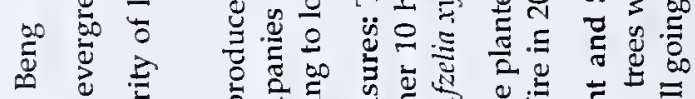

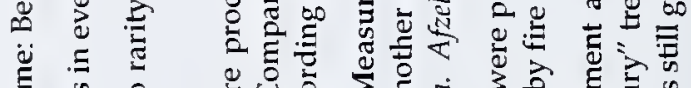

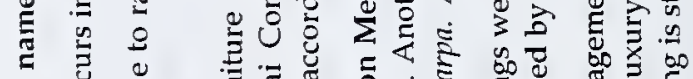

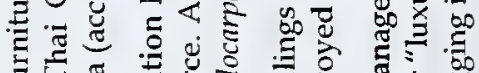

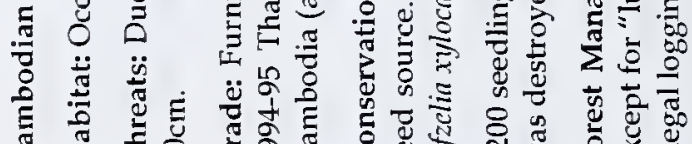

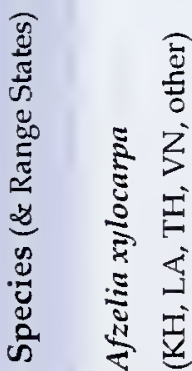

竞毫

든 


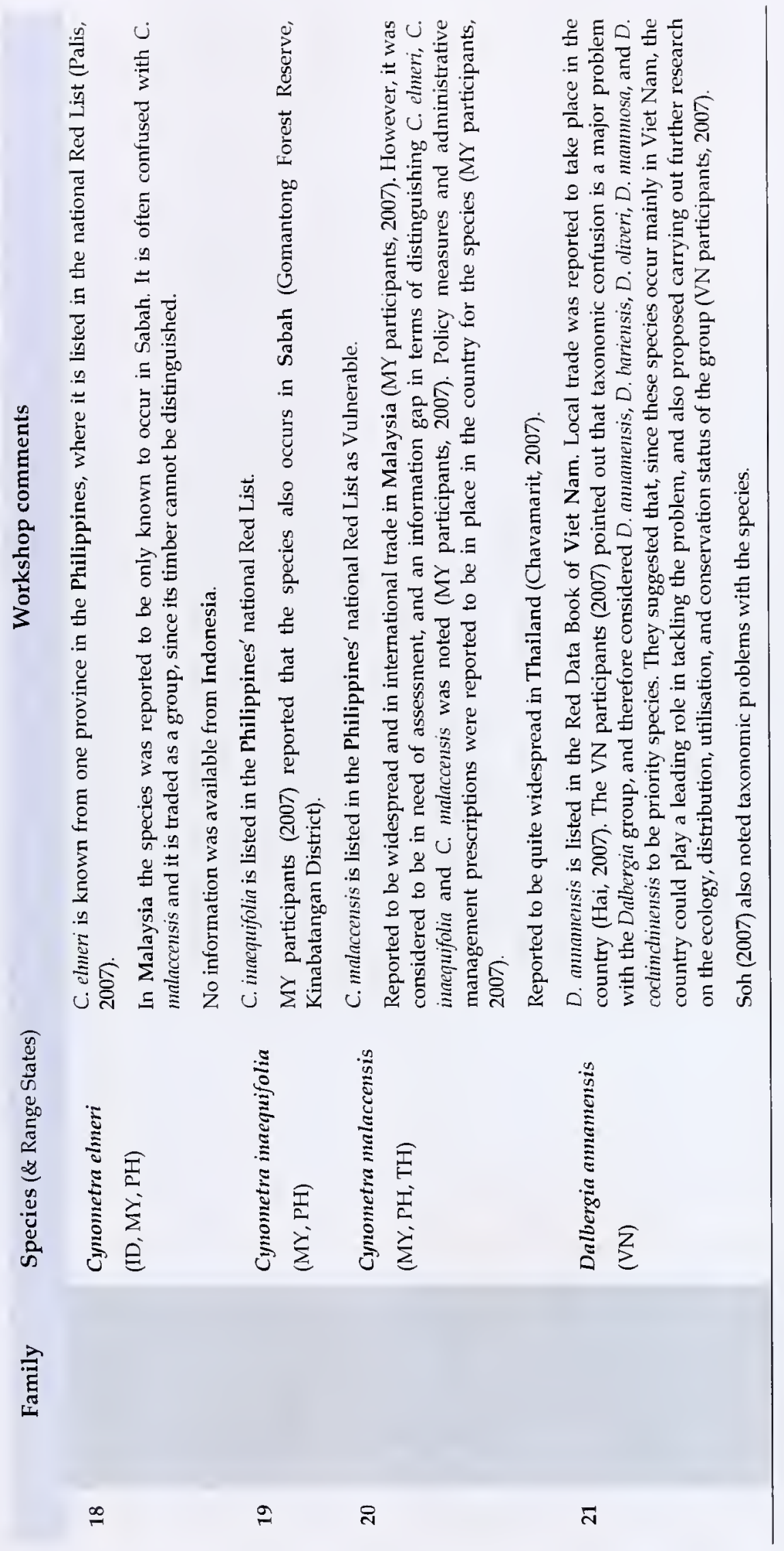

$\tilde{\text { ल }}$ 


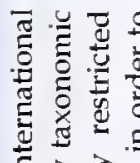

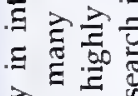

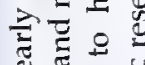

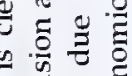

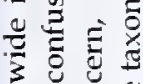

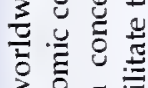

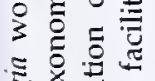

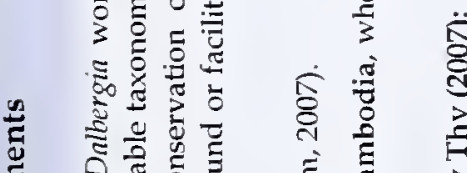

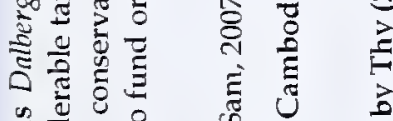

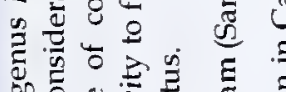

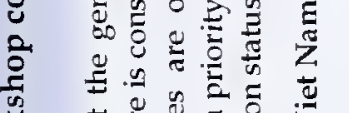

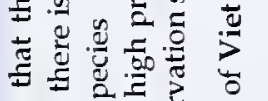

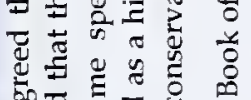

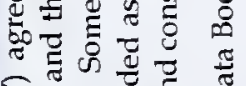

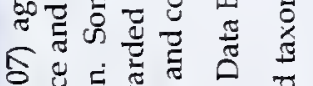

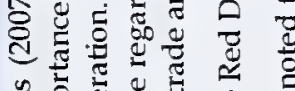

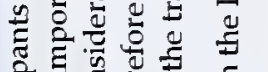
늘

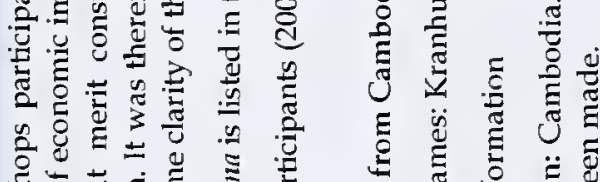

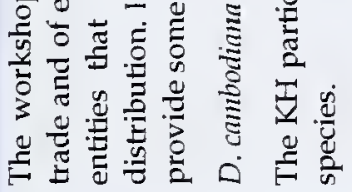

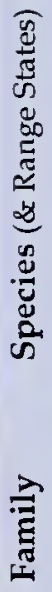

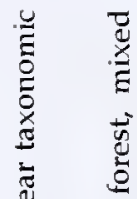

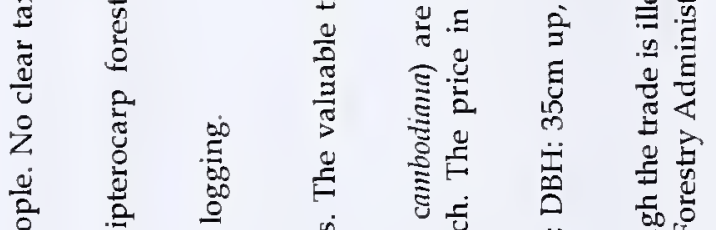

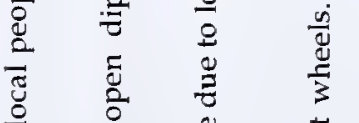

茶

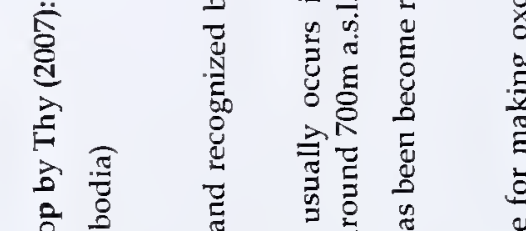

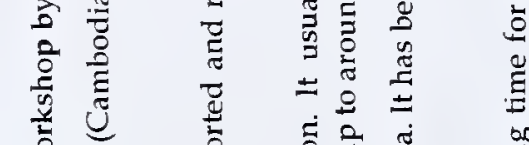

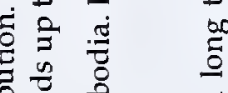

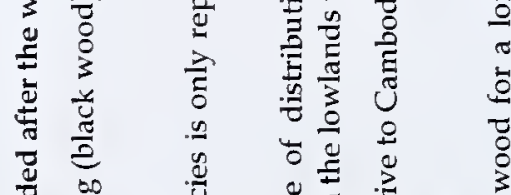

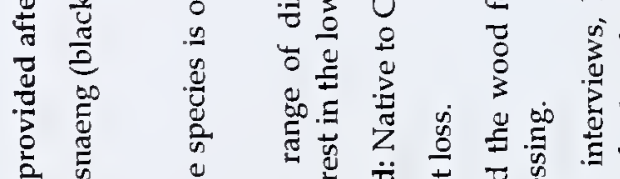

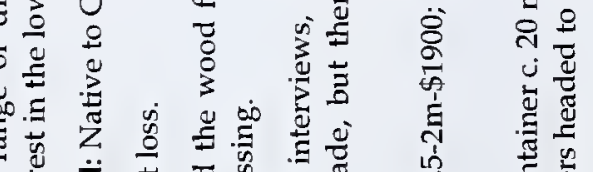

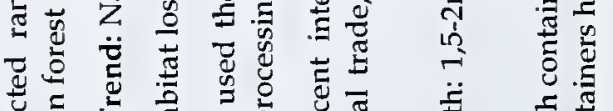

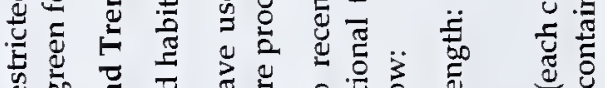

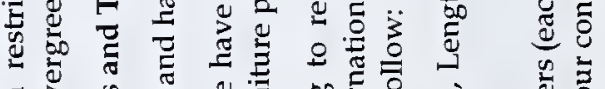

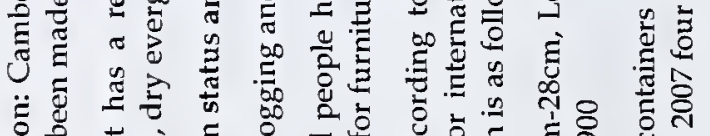

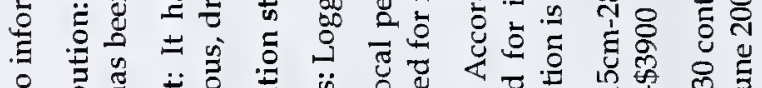

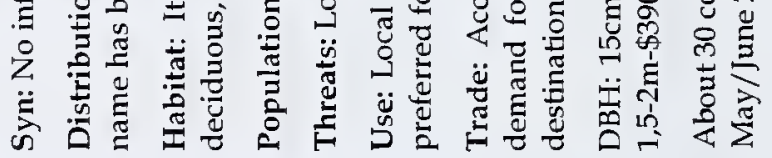




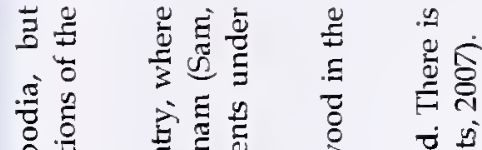

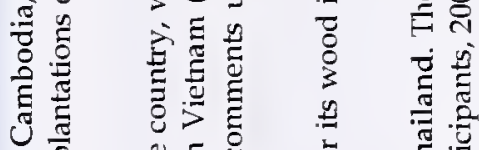

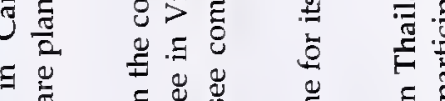

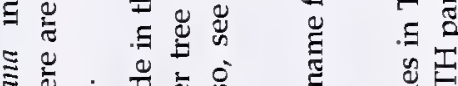

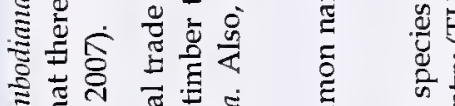

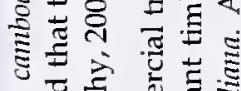

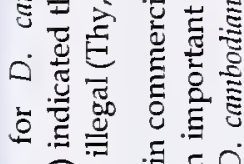

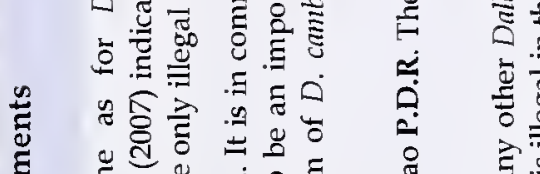

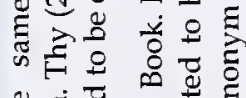

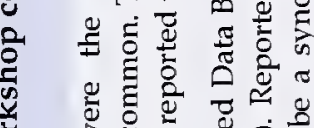

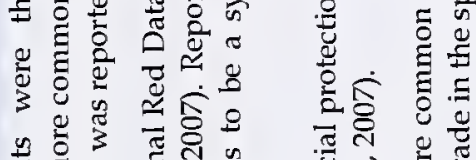

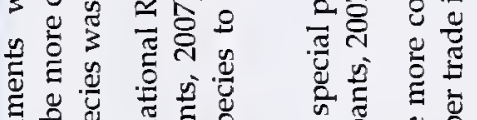

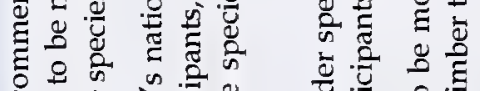

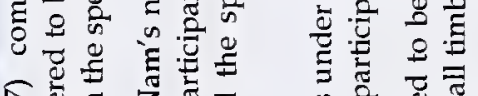
क्ष

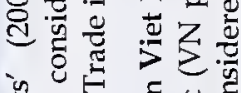

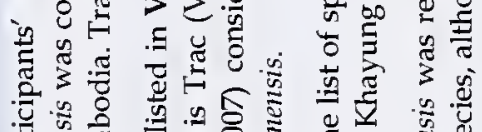

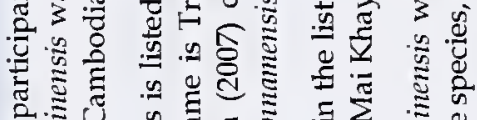

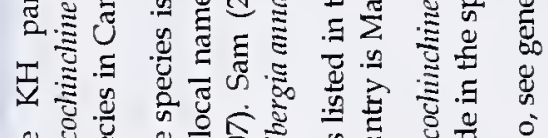

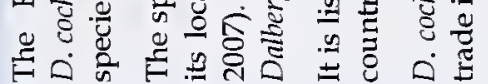

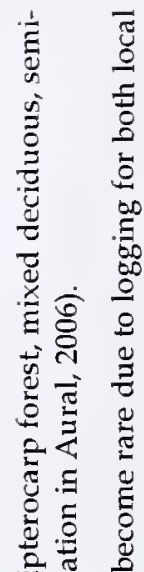

的

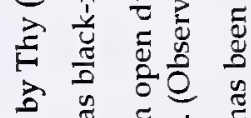

政

政

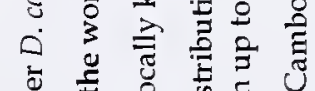

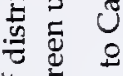

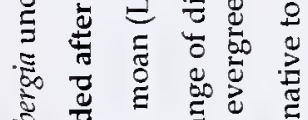

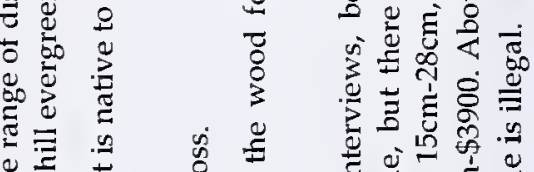

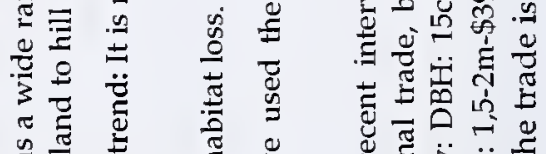

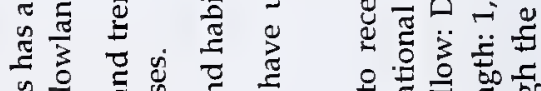

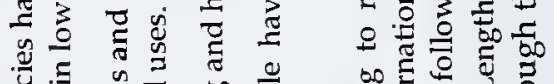

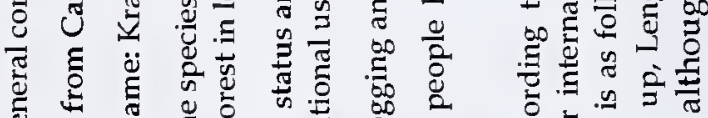
党

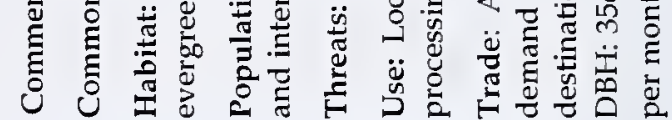

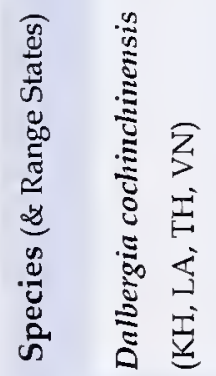

$\underset{\text { 胥 }}{\mathbb{Z}}$ 


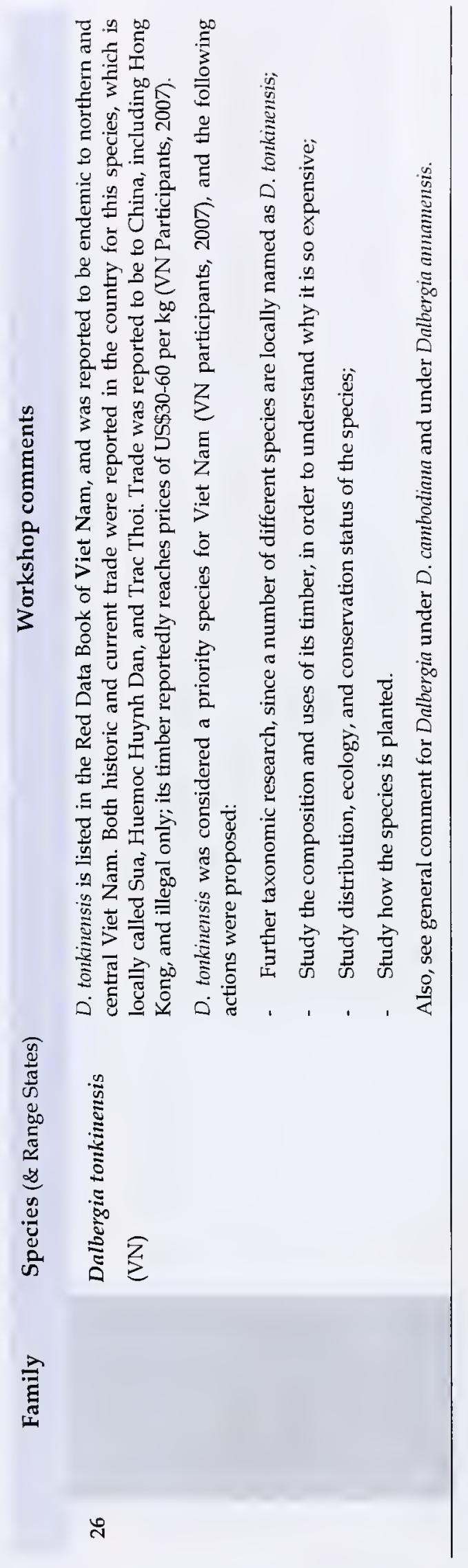




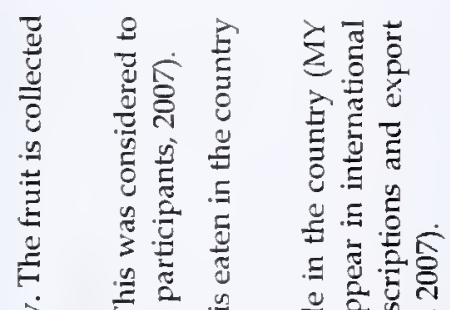

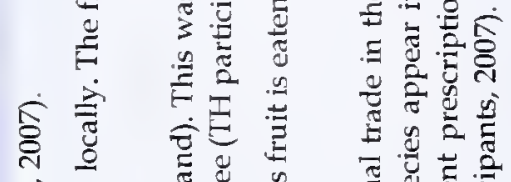

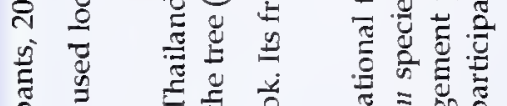

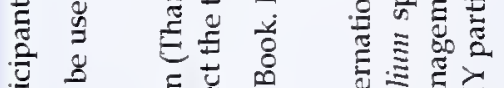

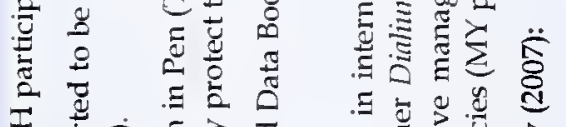

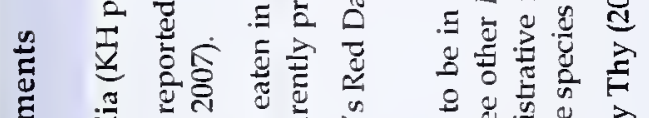

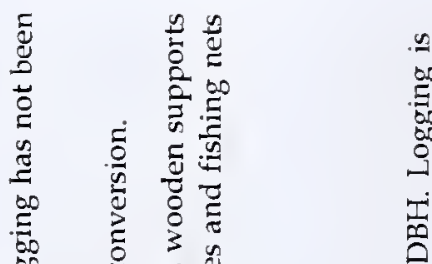

है

อ

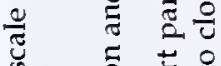

品

๘

ปั.

节育

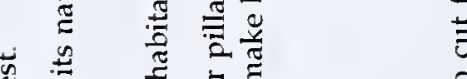

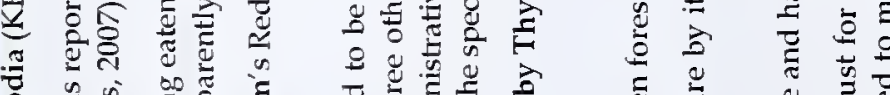

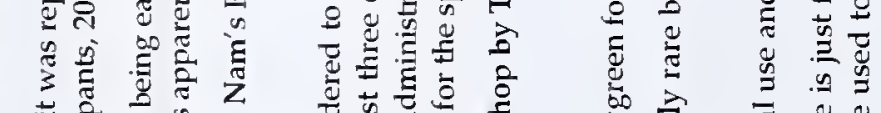

త

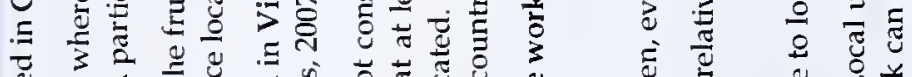

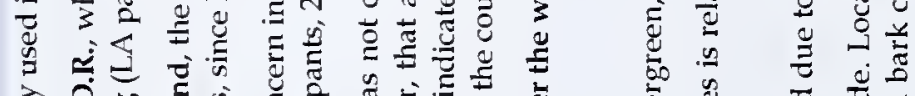

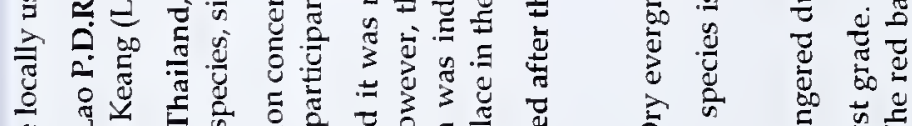

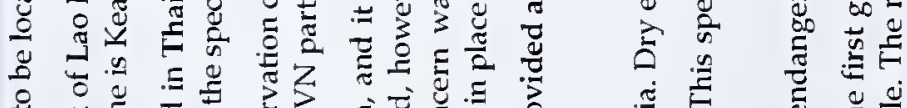

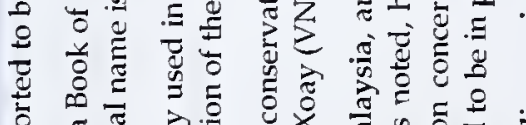

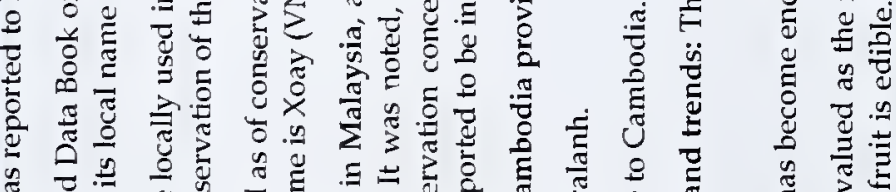

莺滎

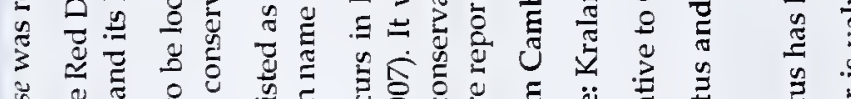

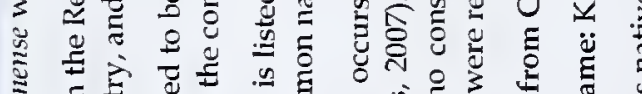

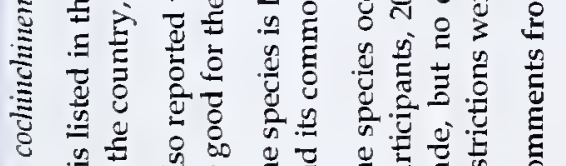

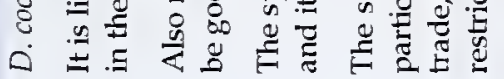

空

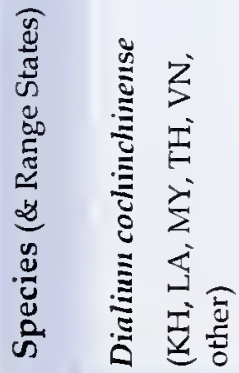

常 


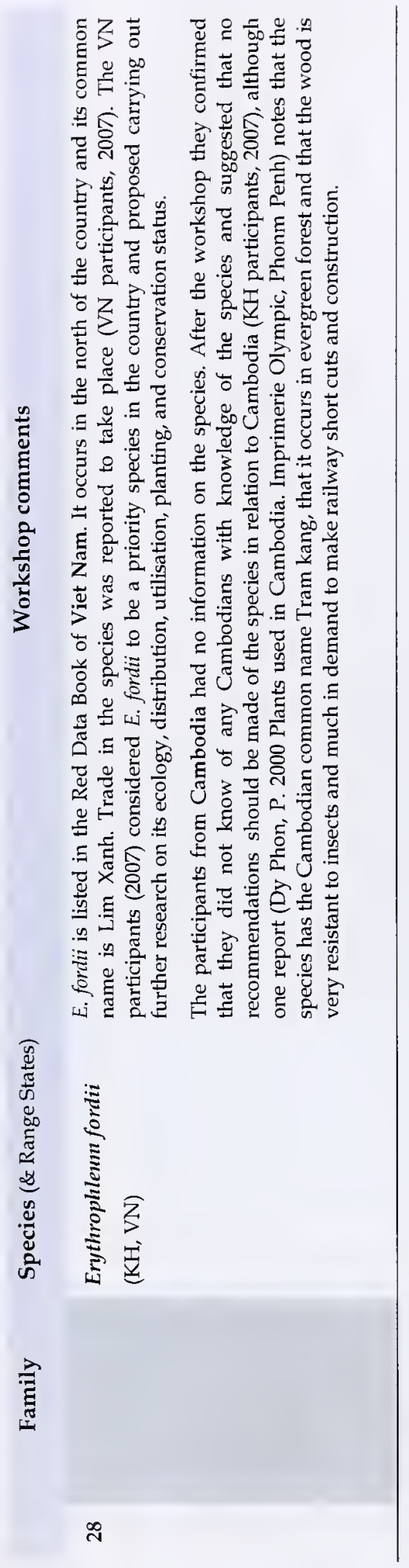




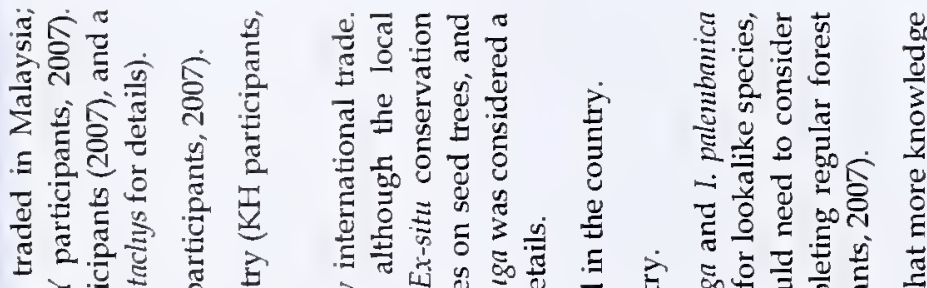

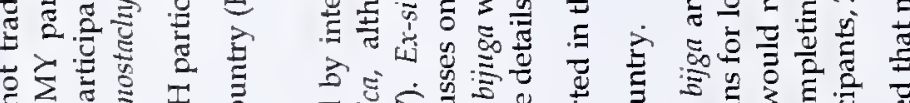

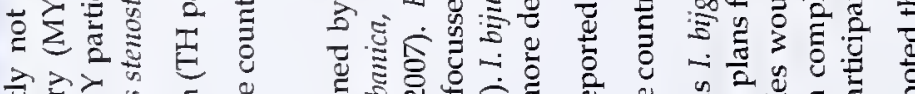

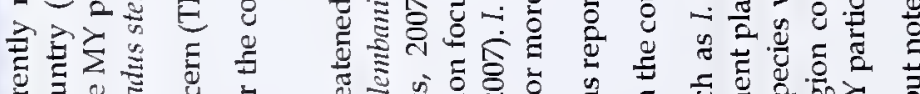

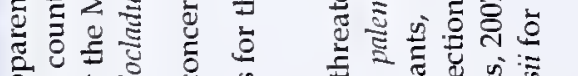
일

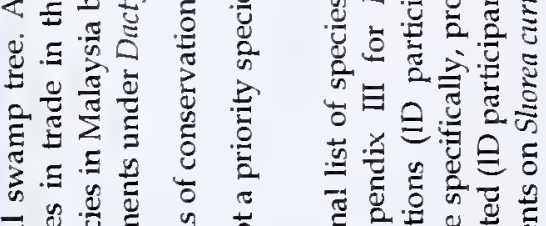

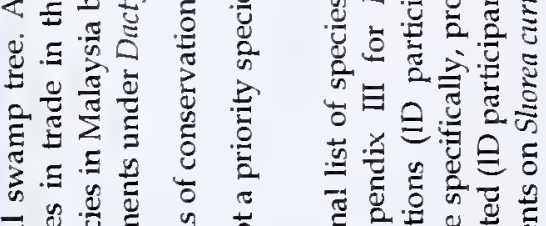

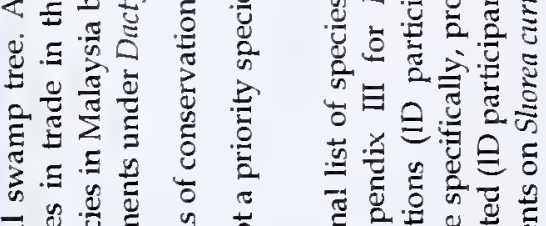

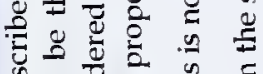

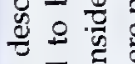

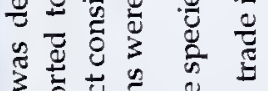

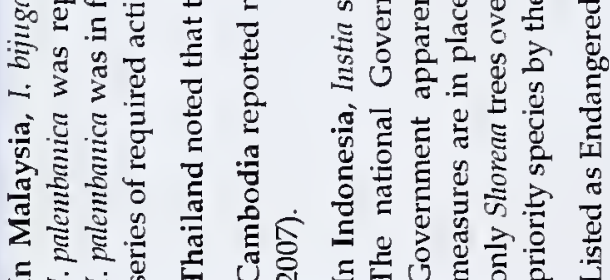

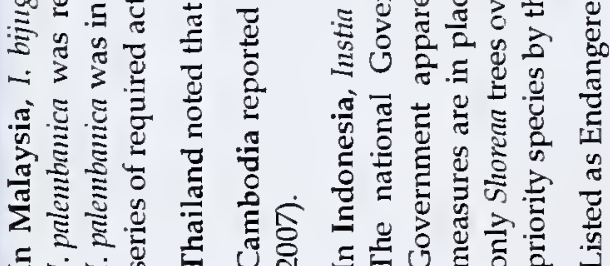

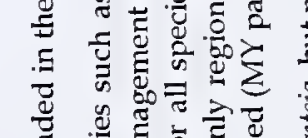

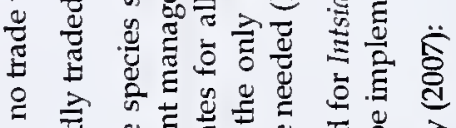

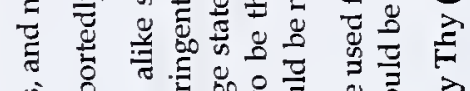
这告

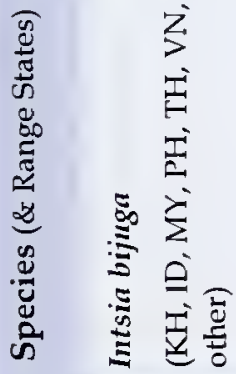

를 


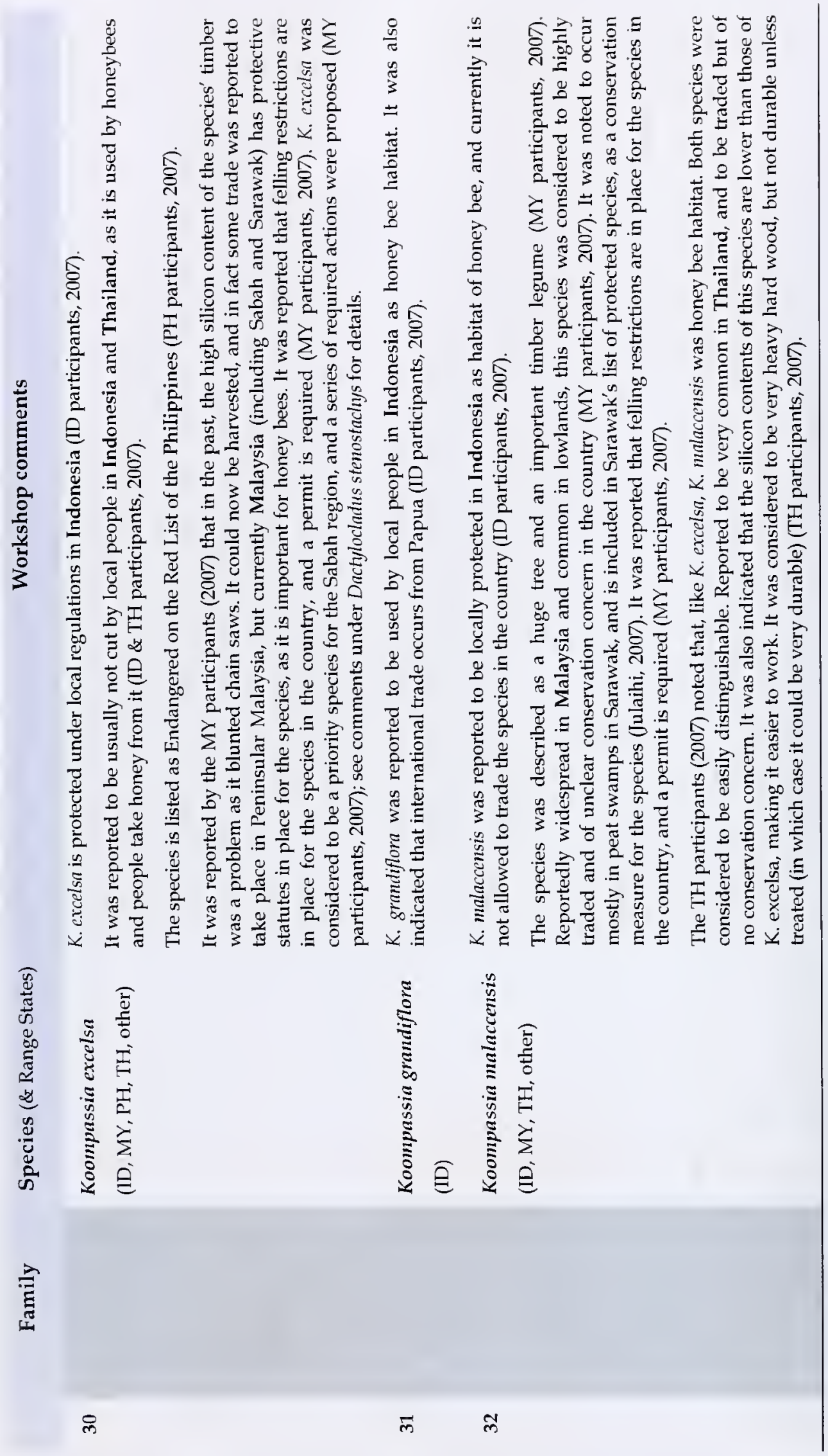

아 


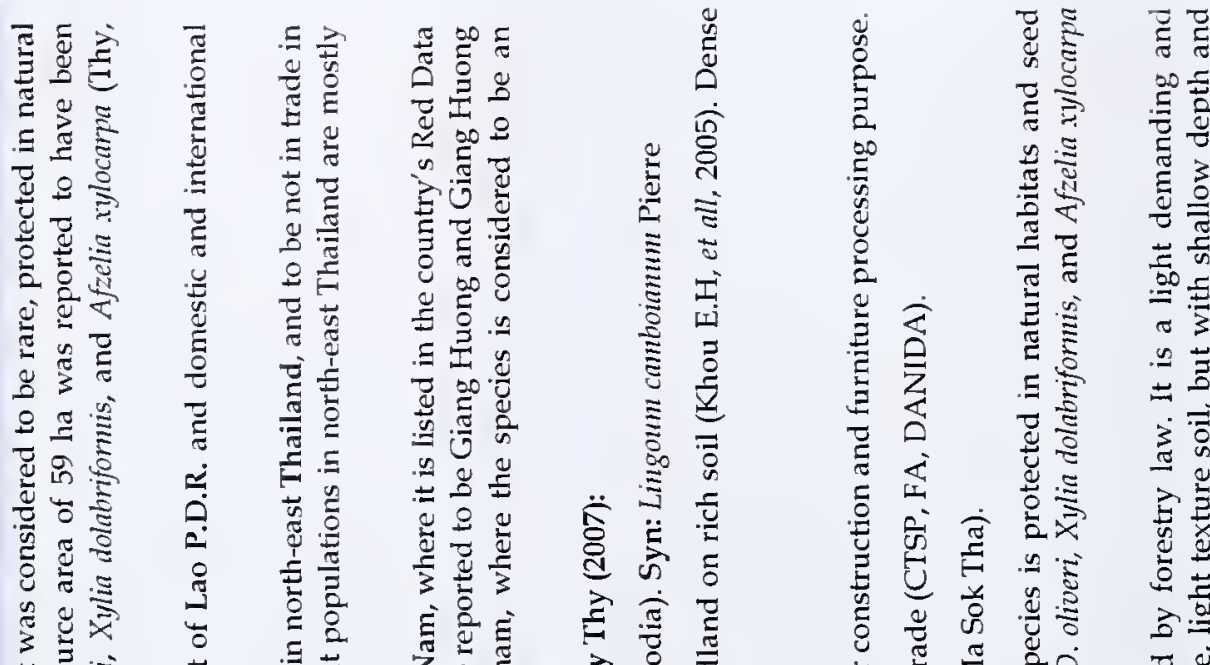

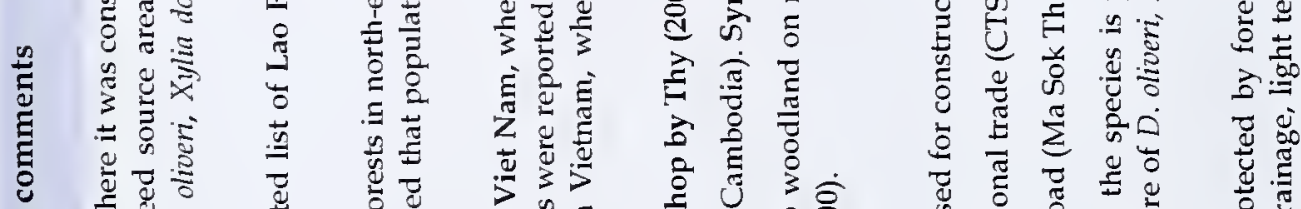

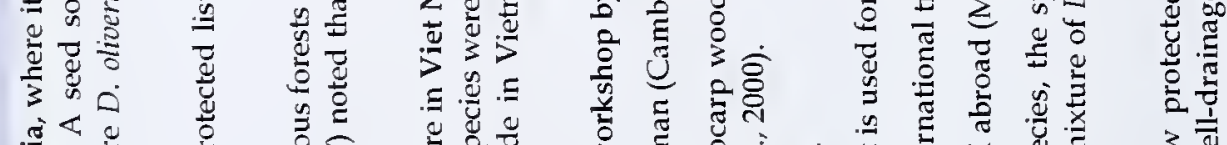

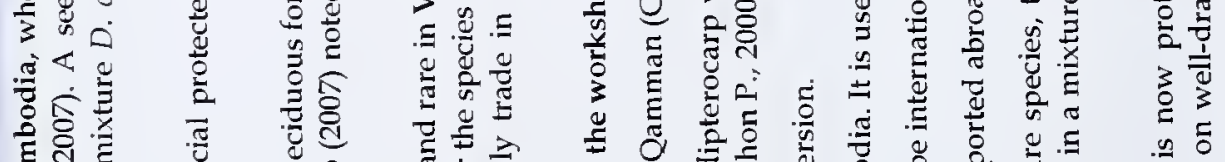

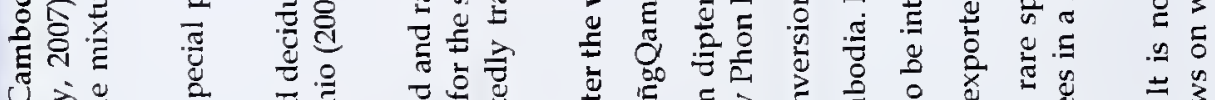

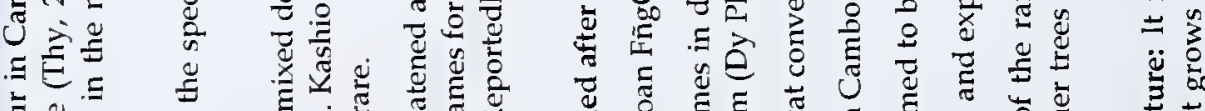

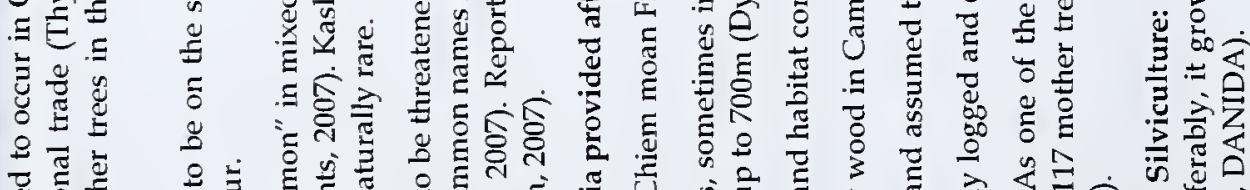

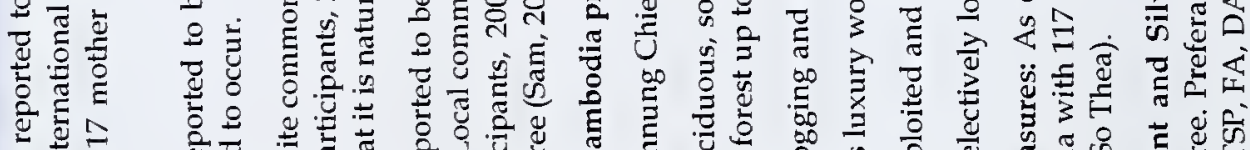

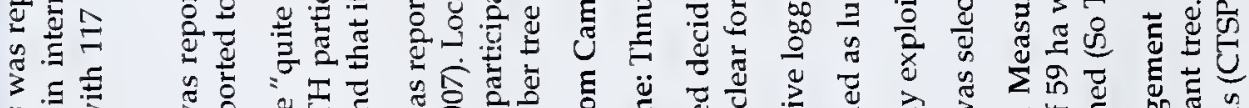

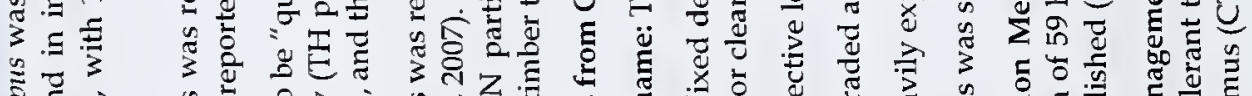

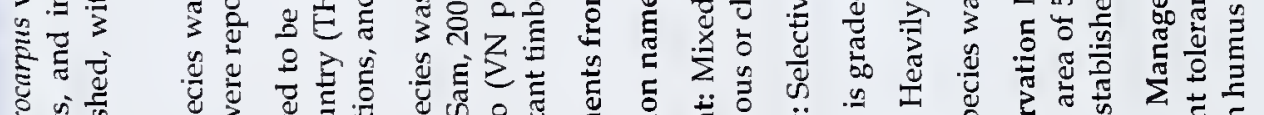

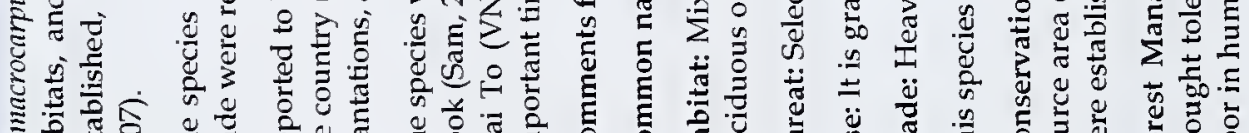

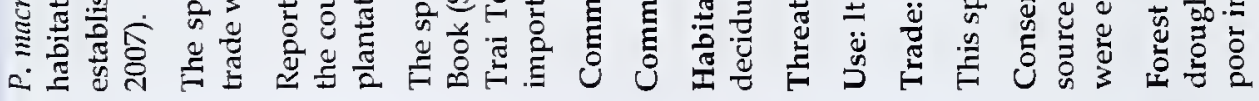

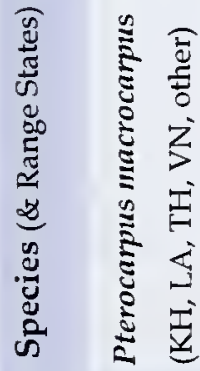

产 


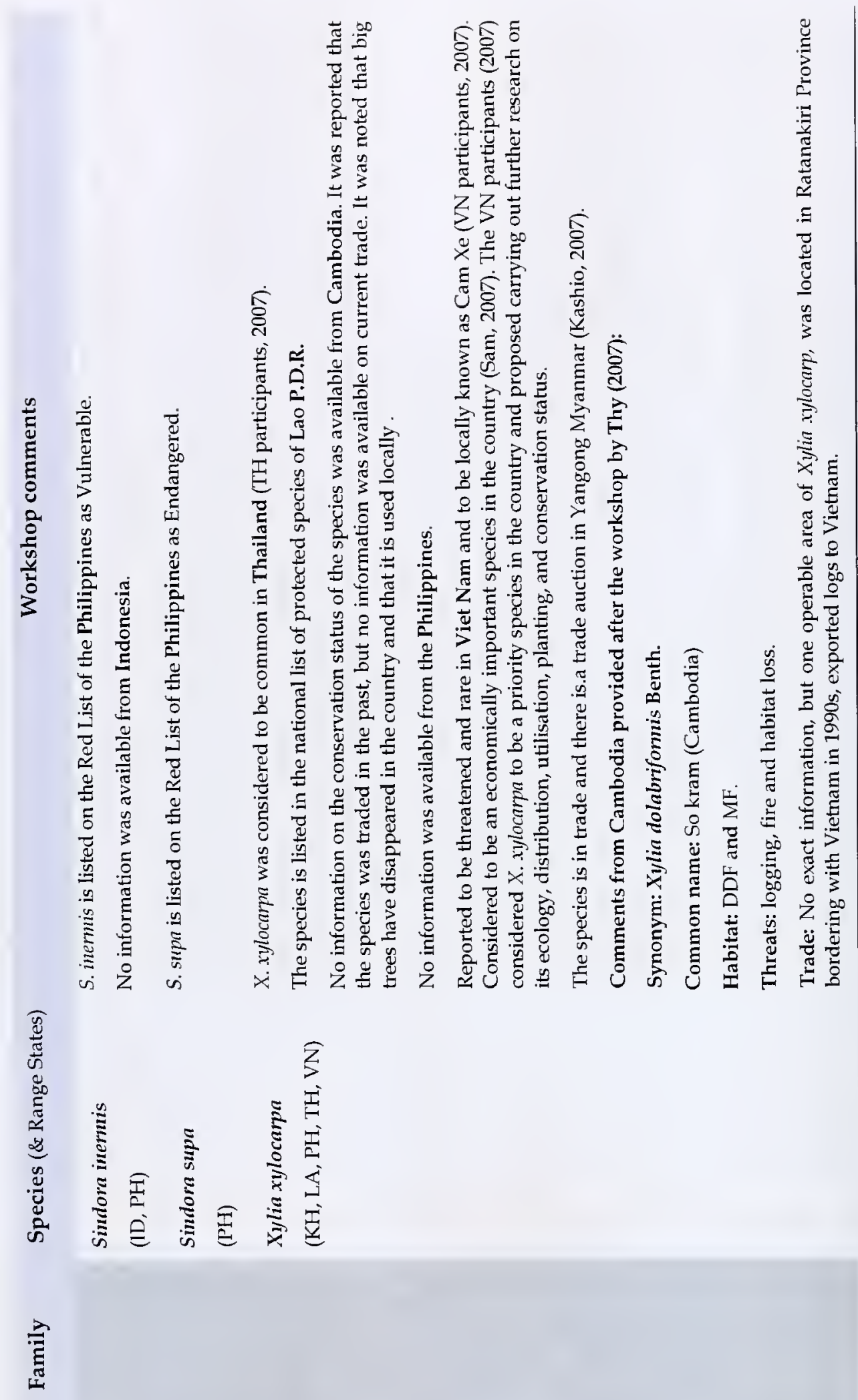

से 


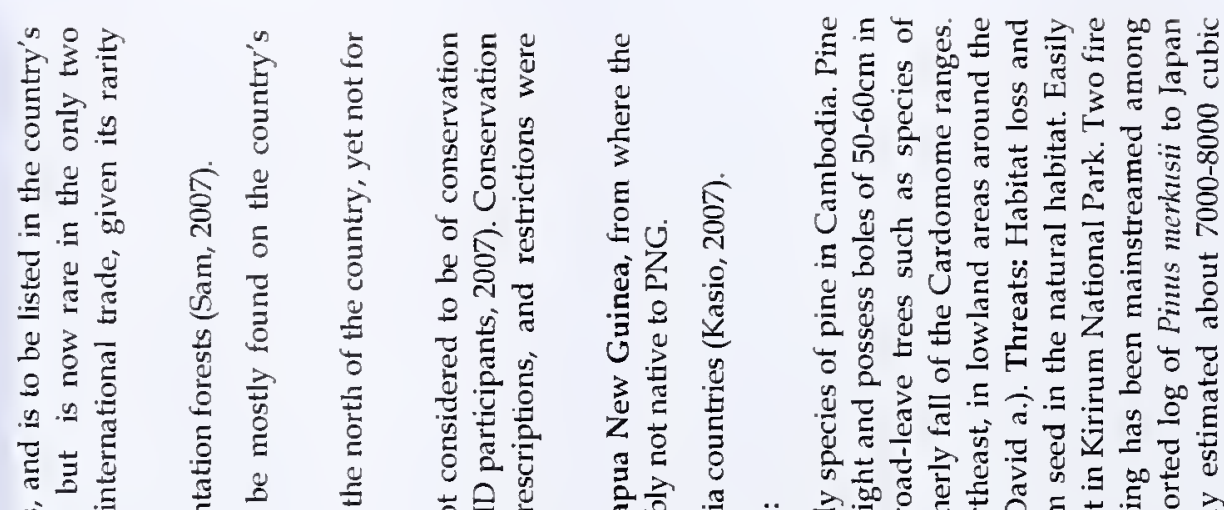

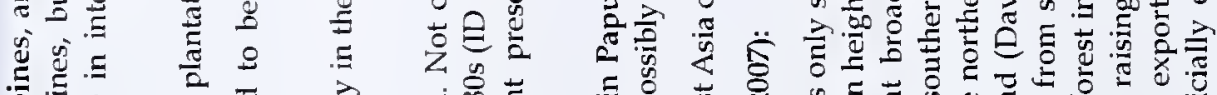

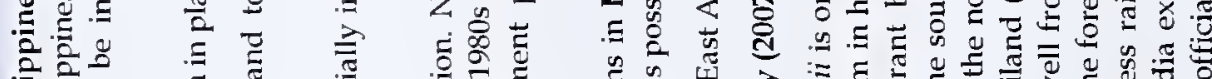

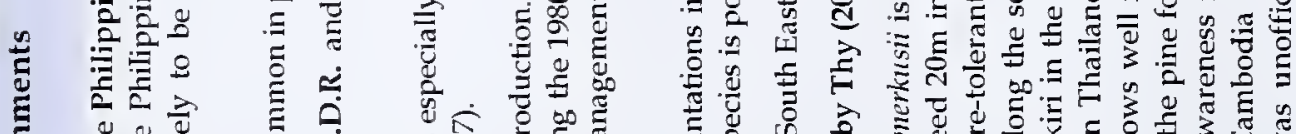

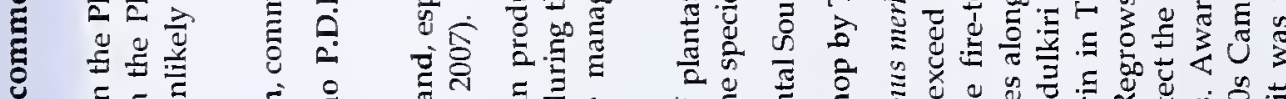

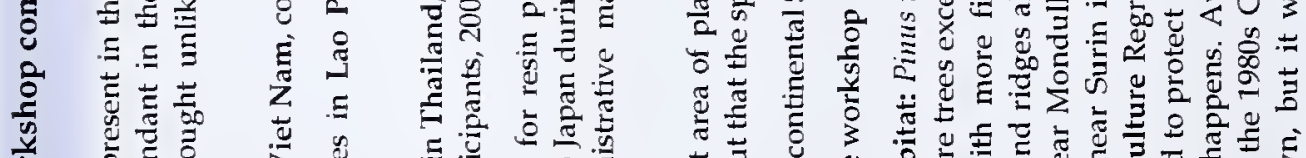

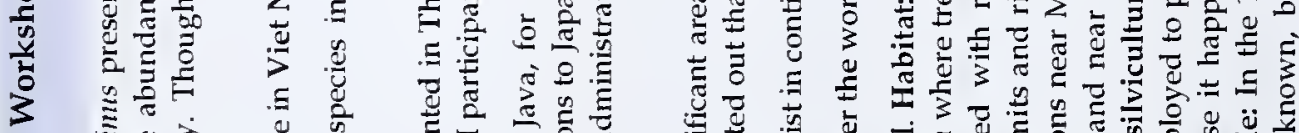

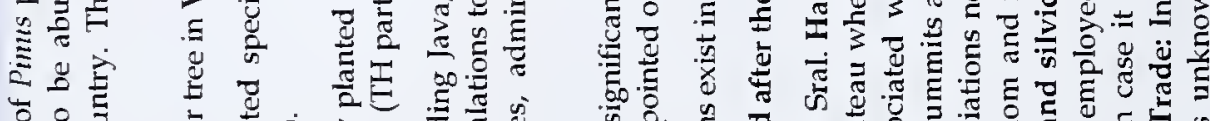

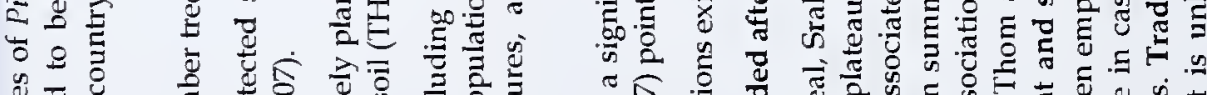

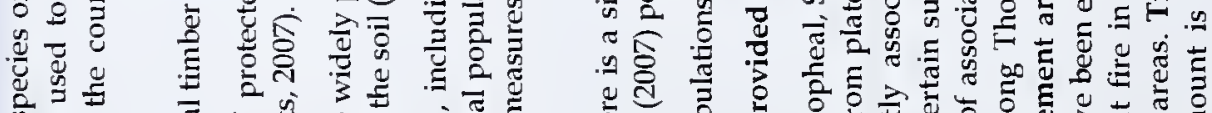

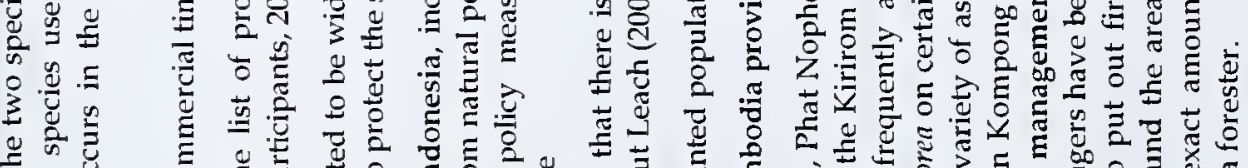

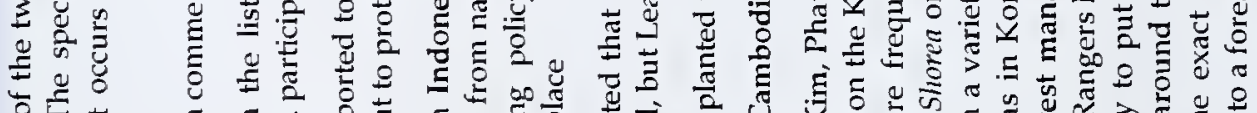

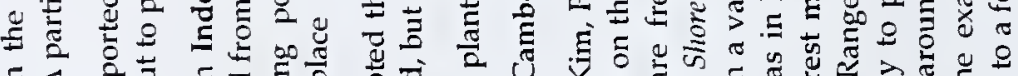

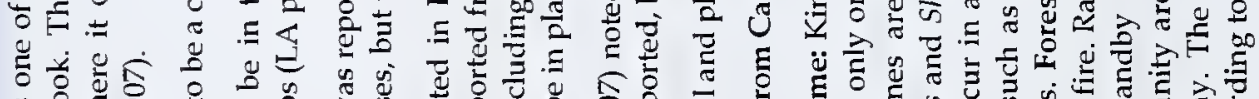

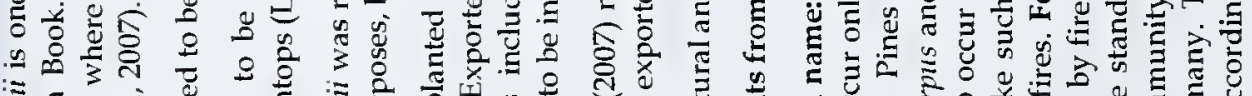

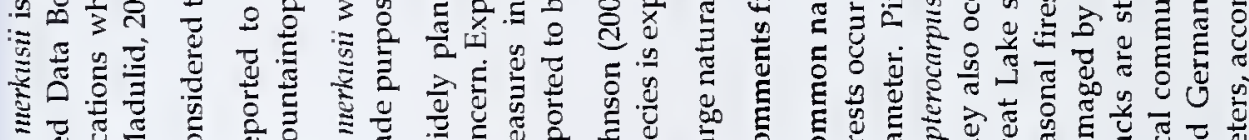

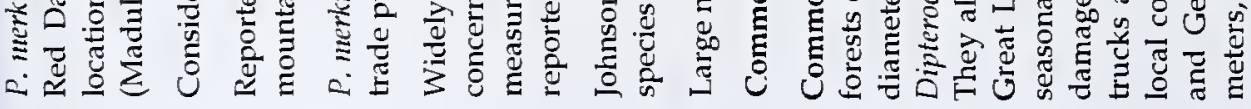

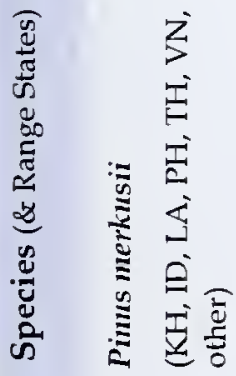

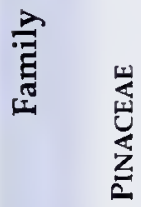




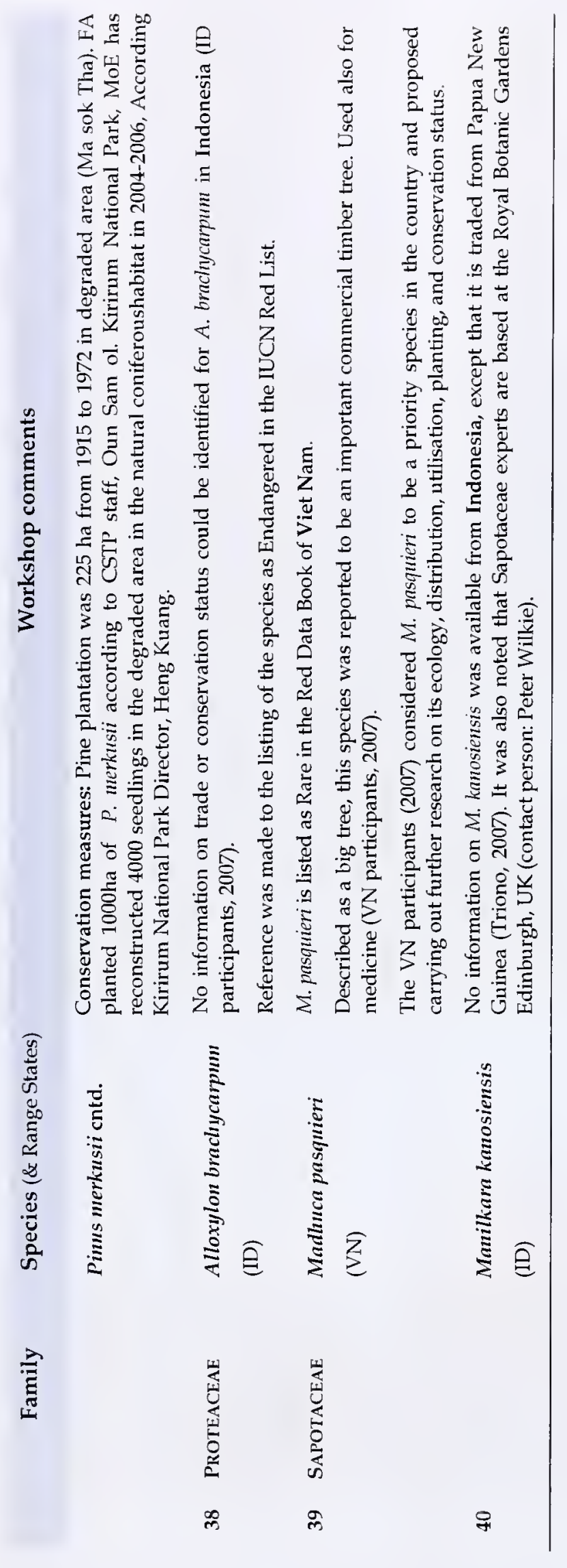

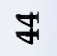




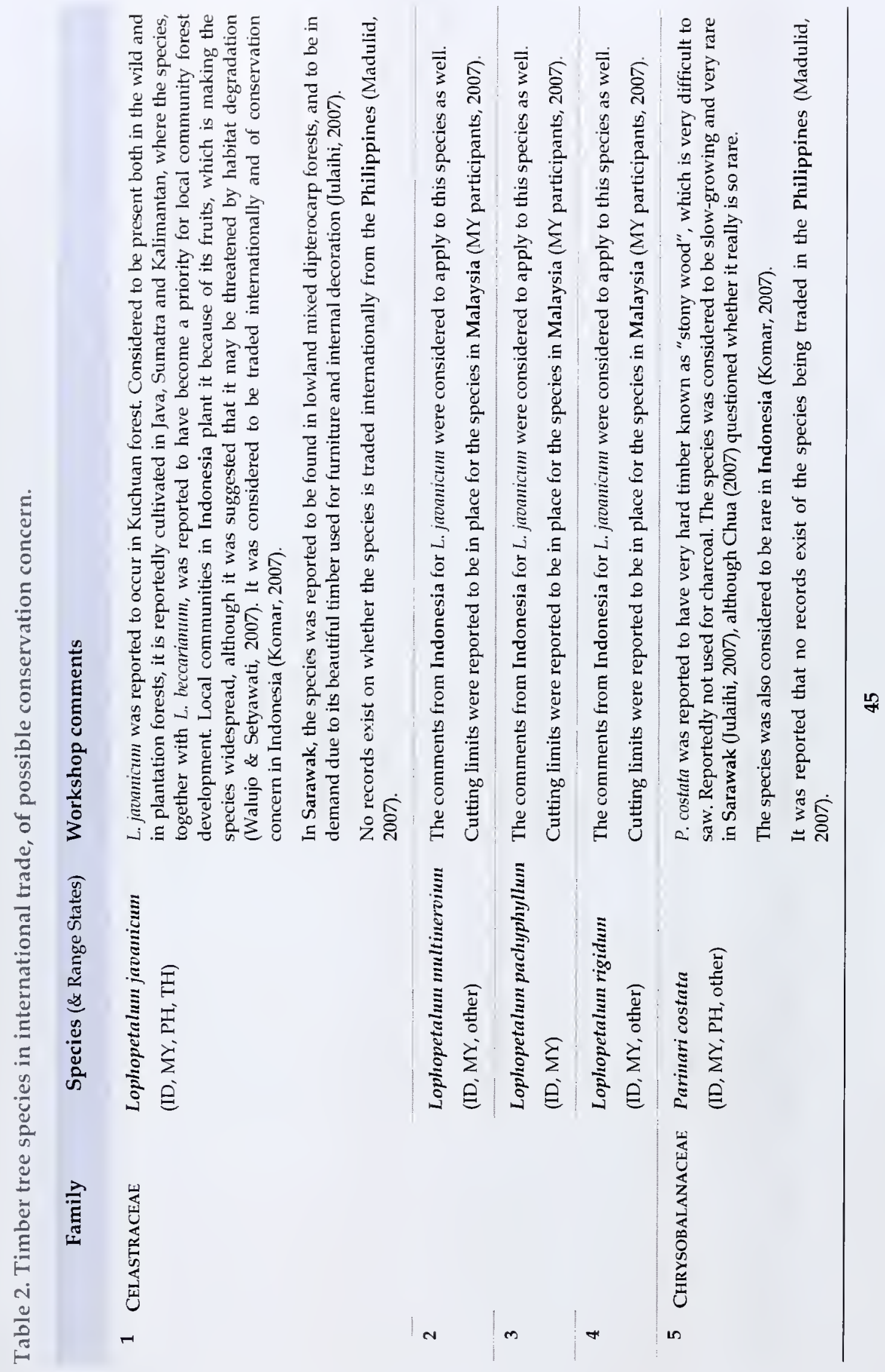




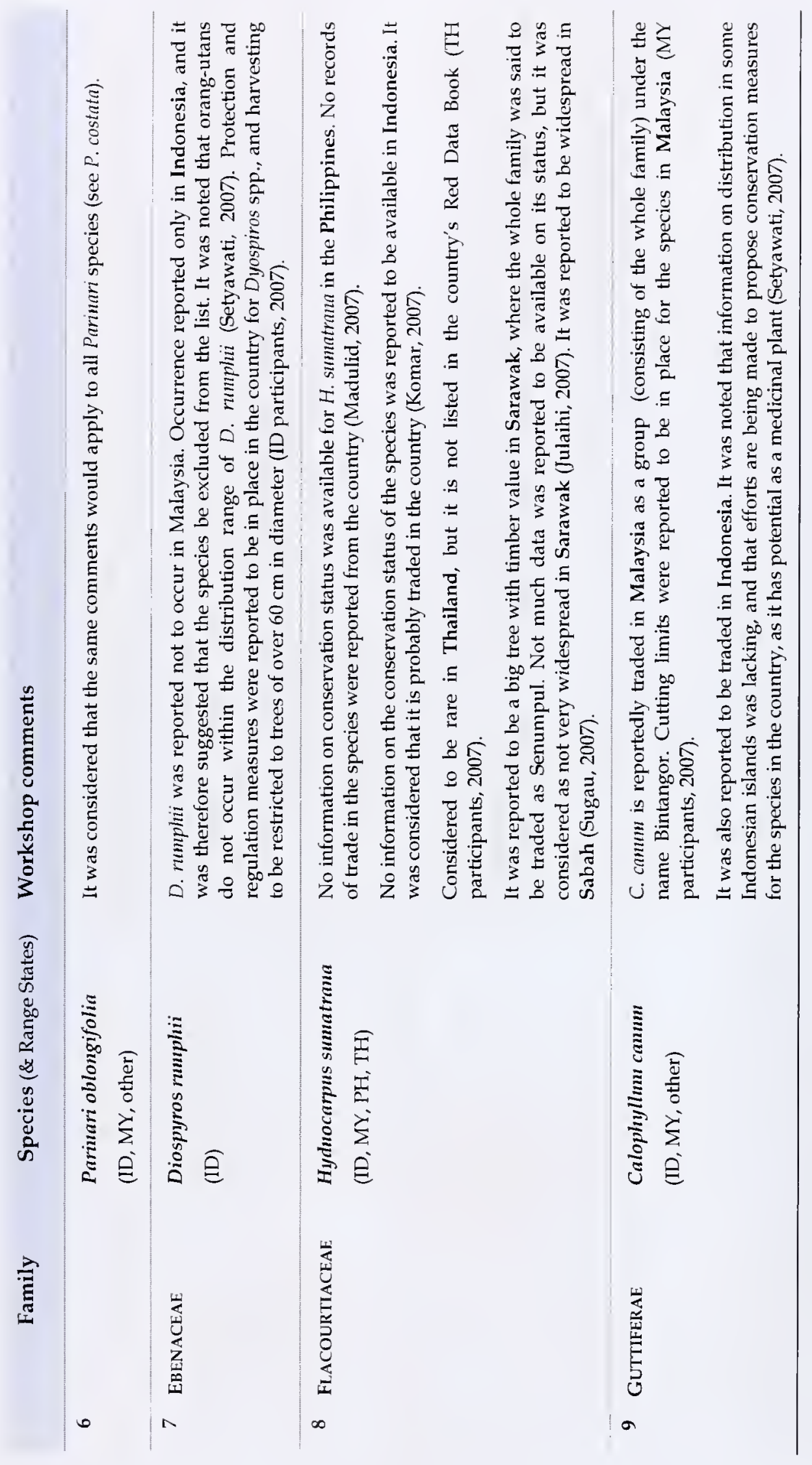




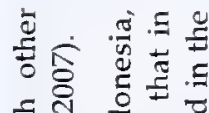

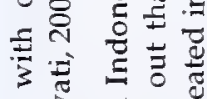

可言

高敦

8

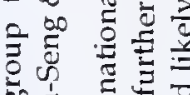

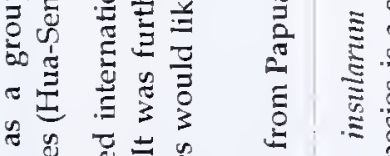

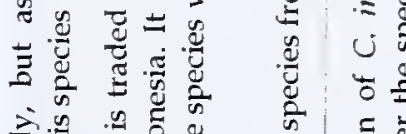

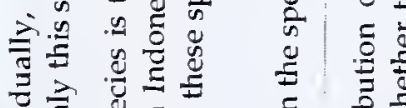

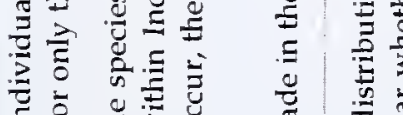

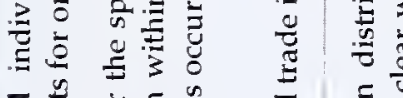

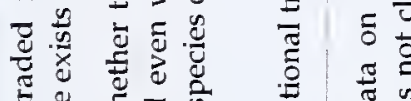

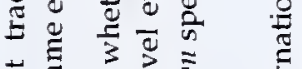

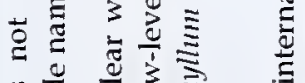

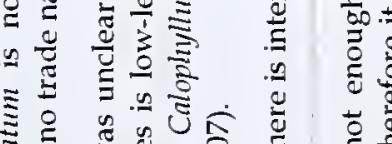

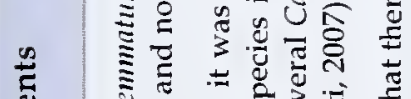

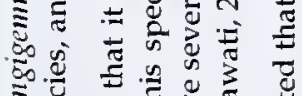

亏气

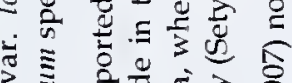

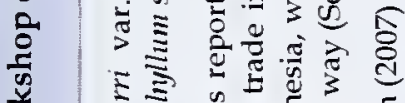

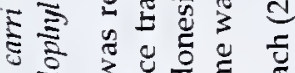

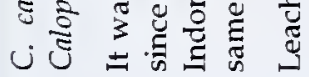

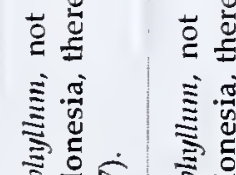

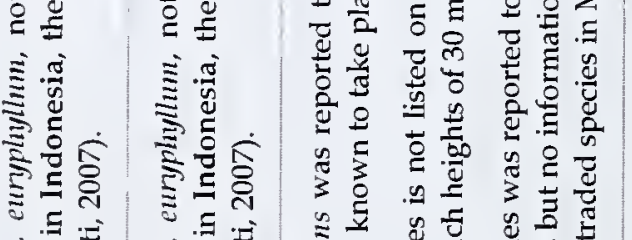

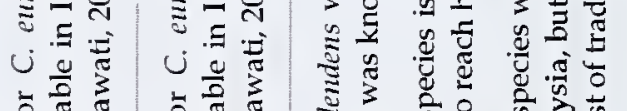

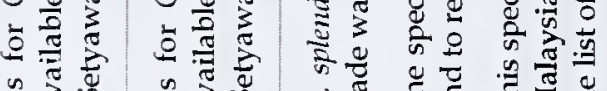

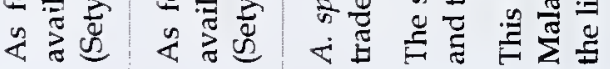
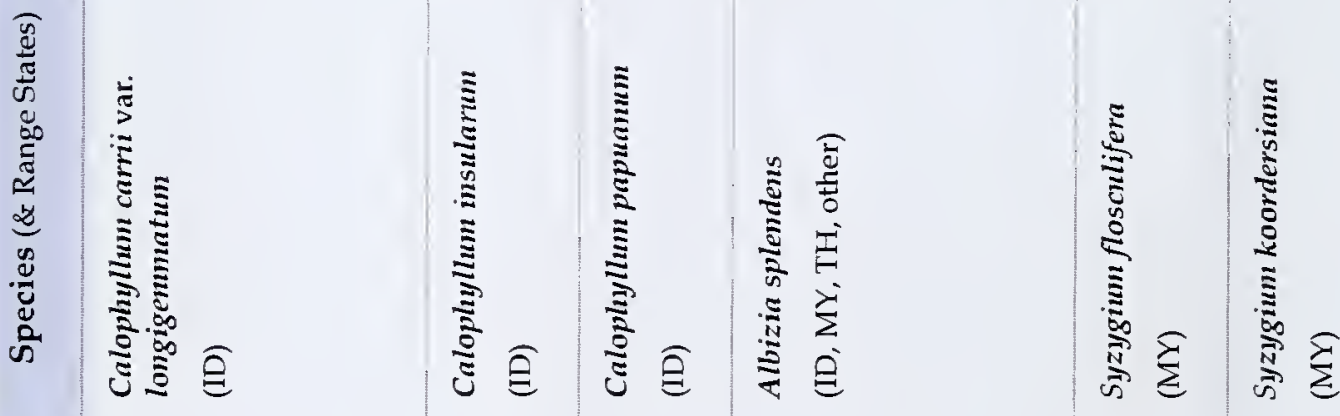

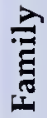
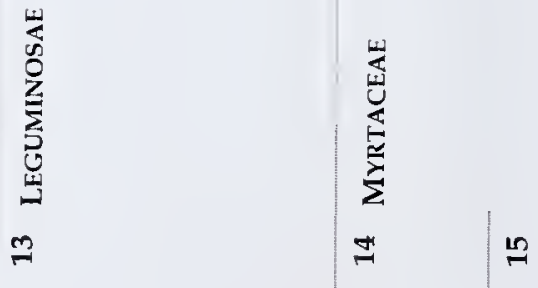


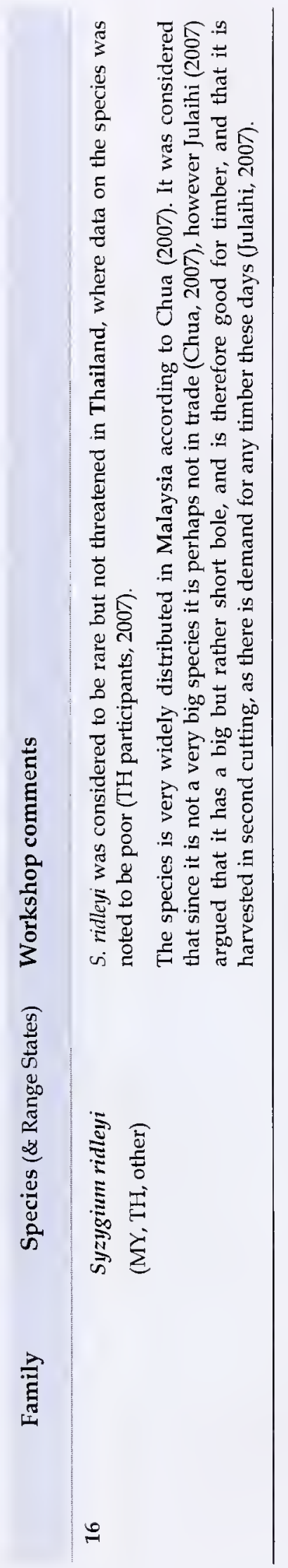




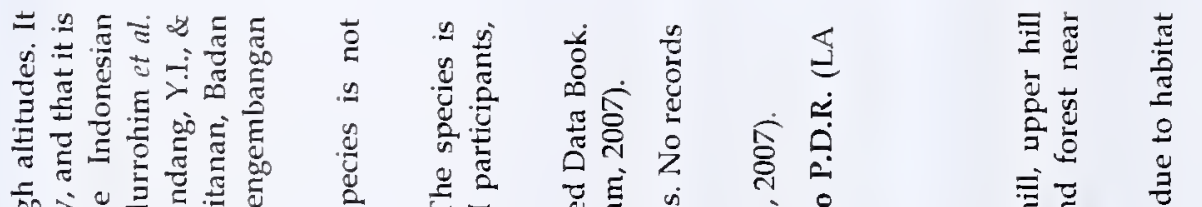

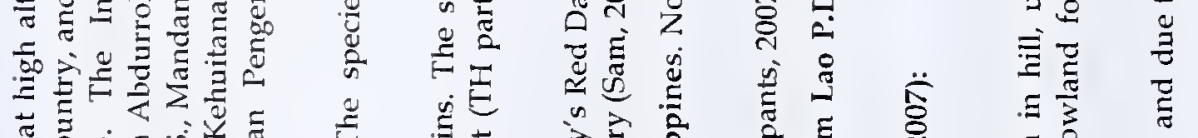

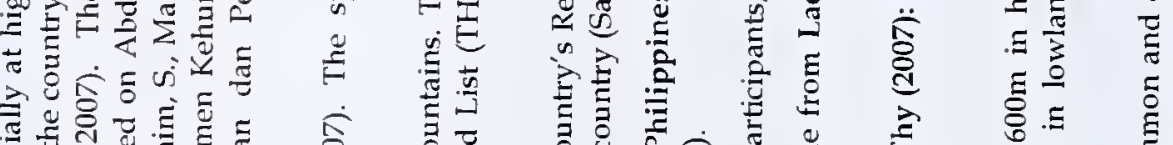

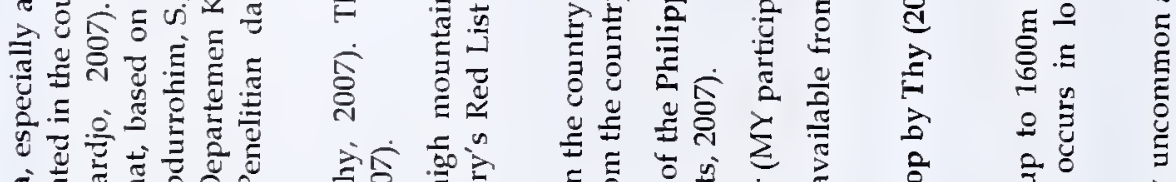

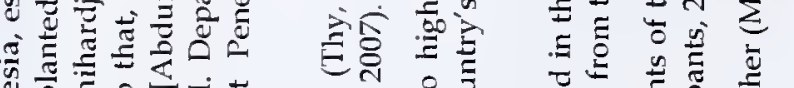

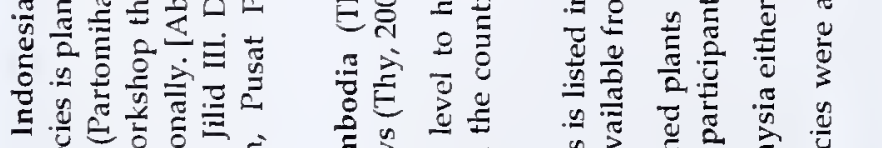

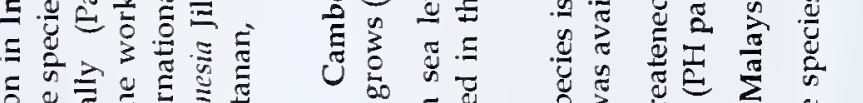

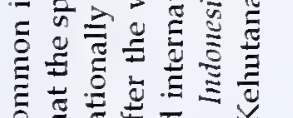

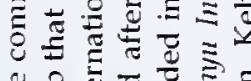

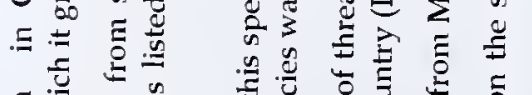

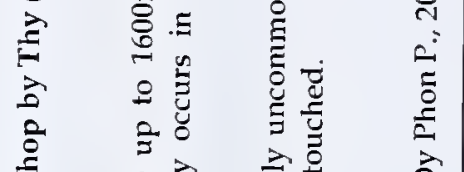

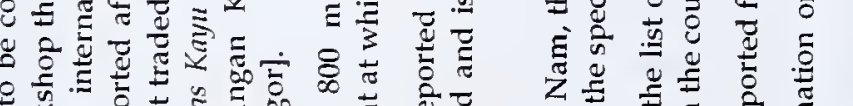

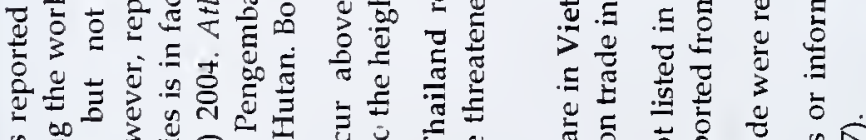

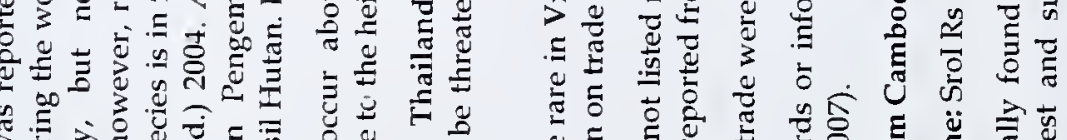

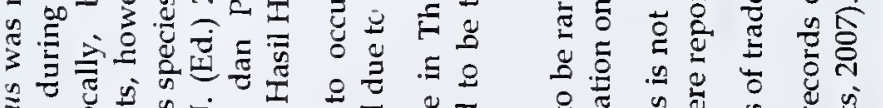

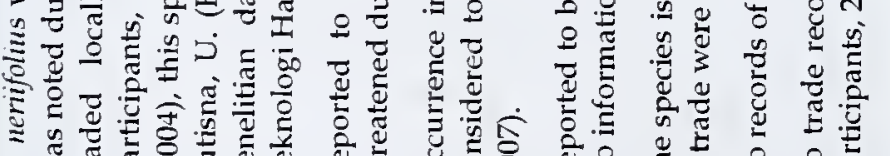

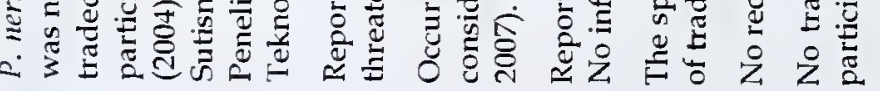

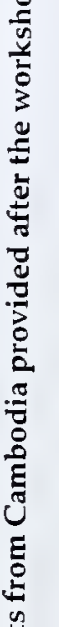

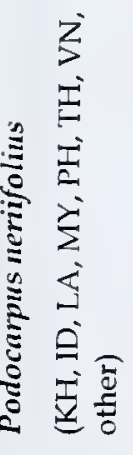

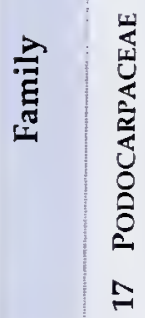




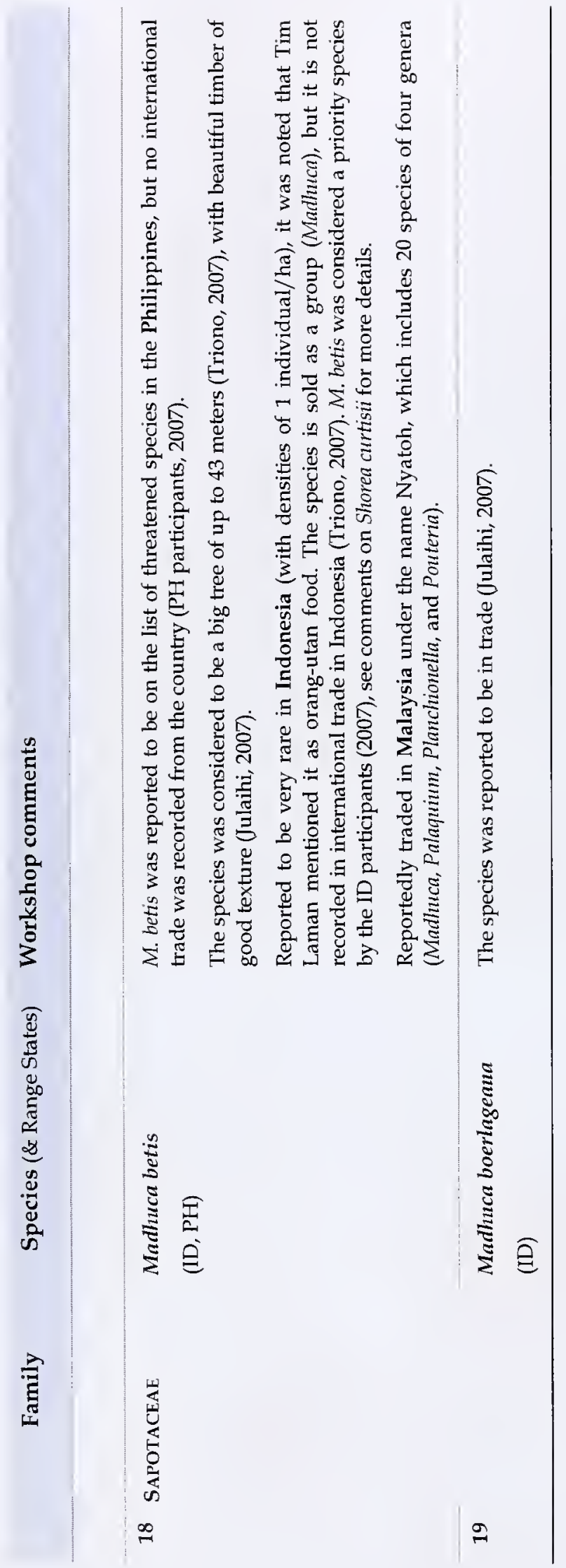

ํํ 


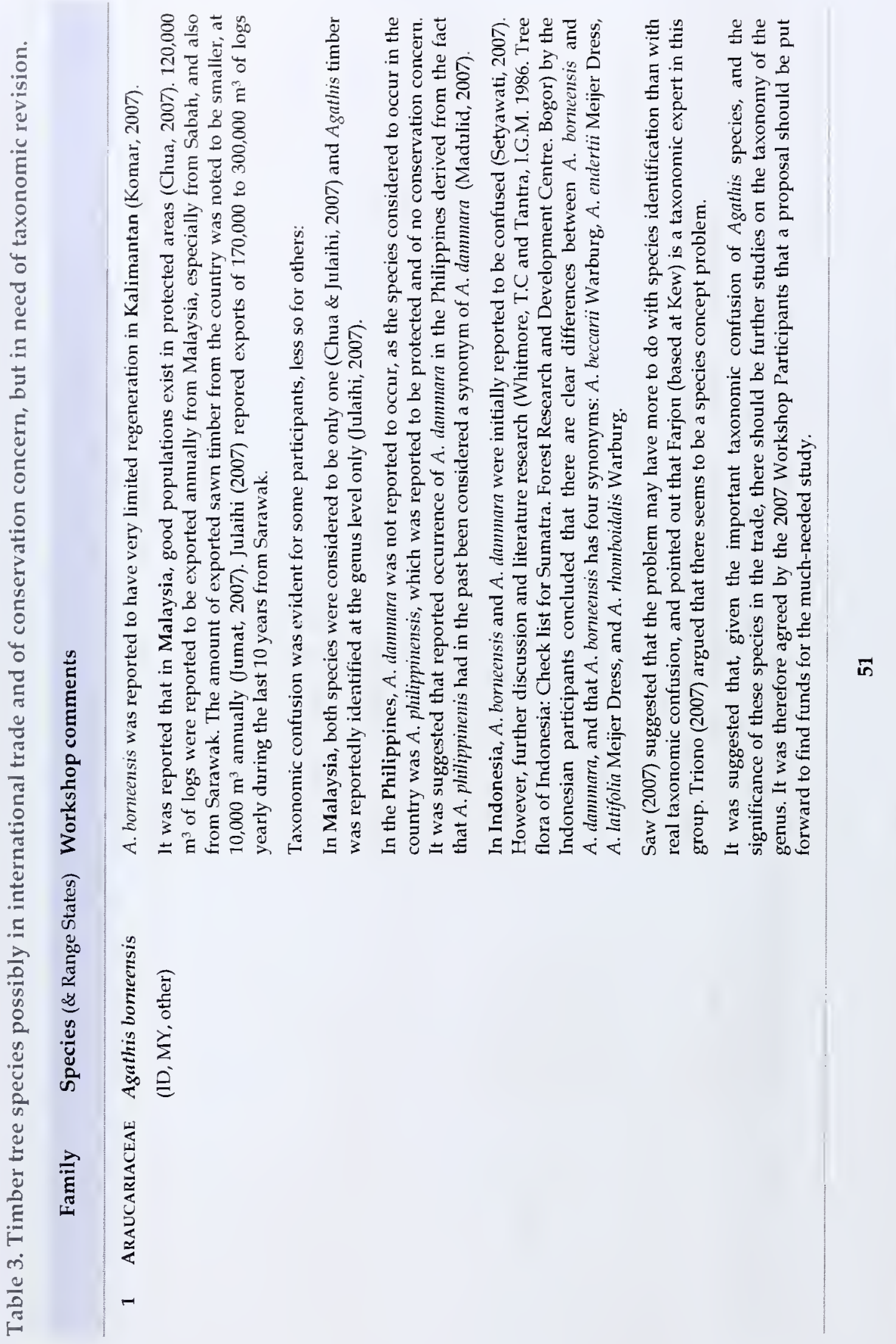




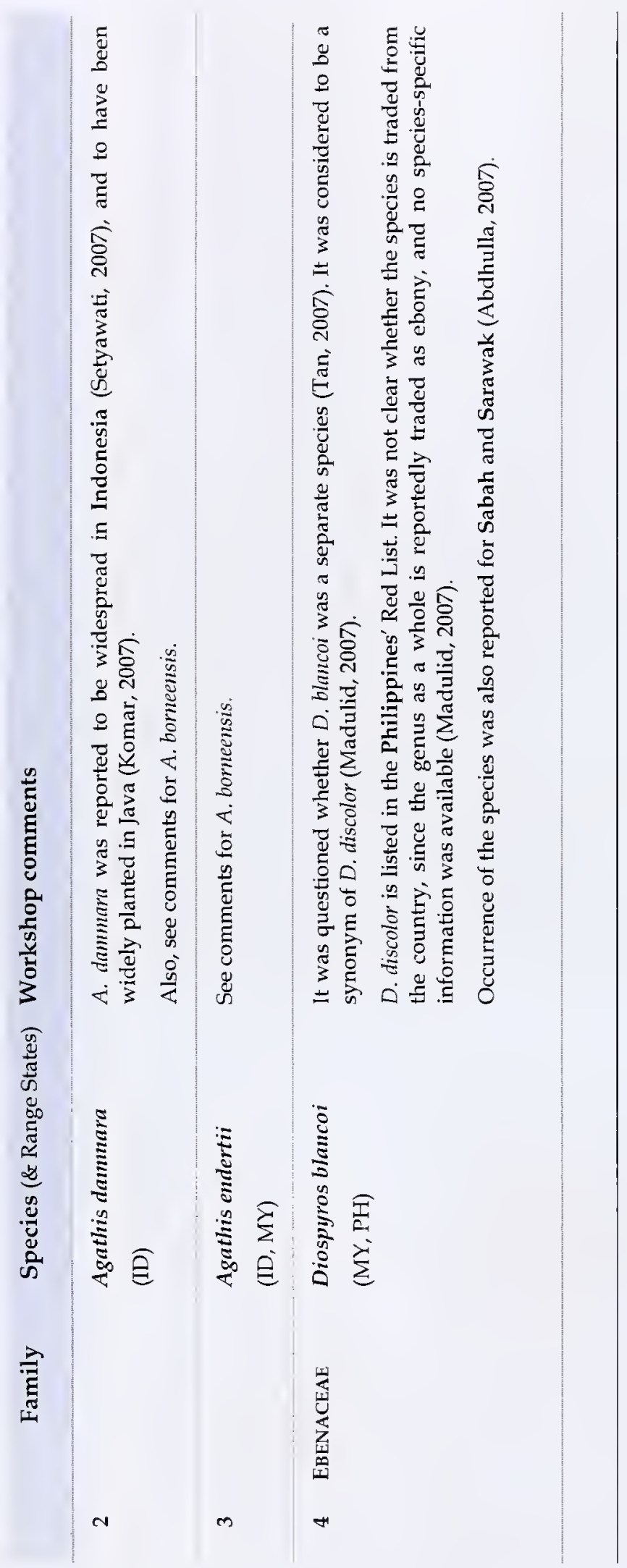

กิ 


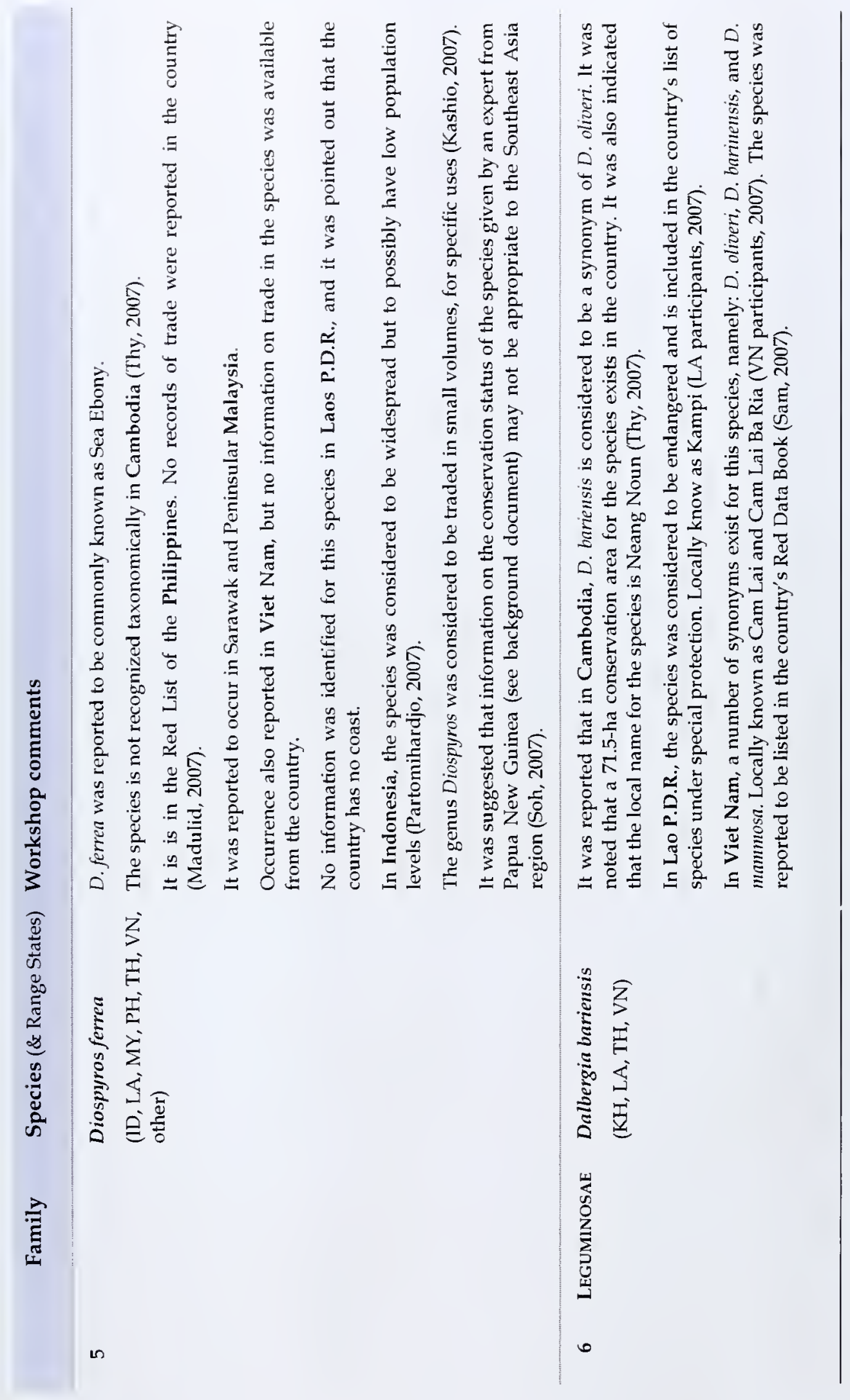

in 


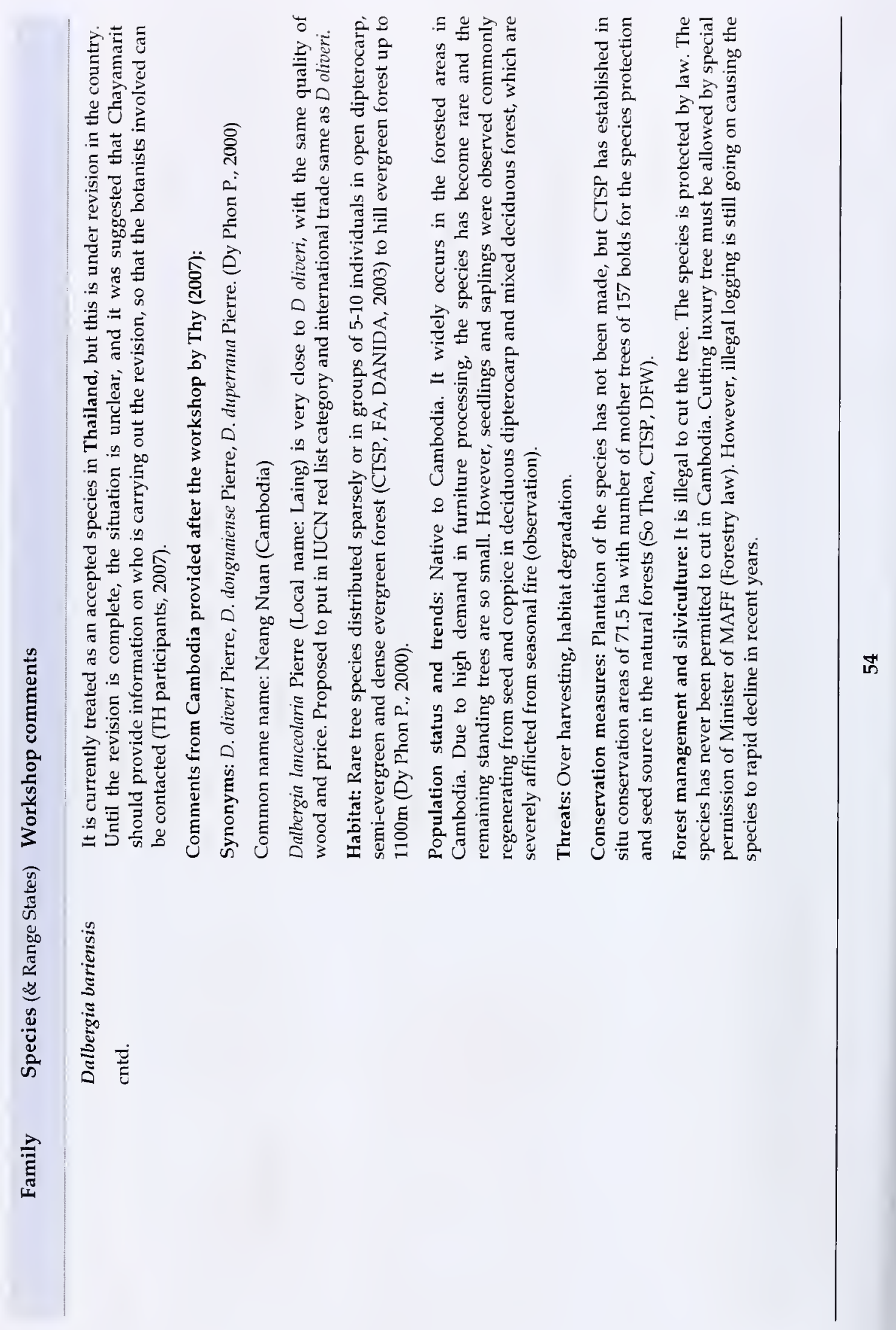




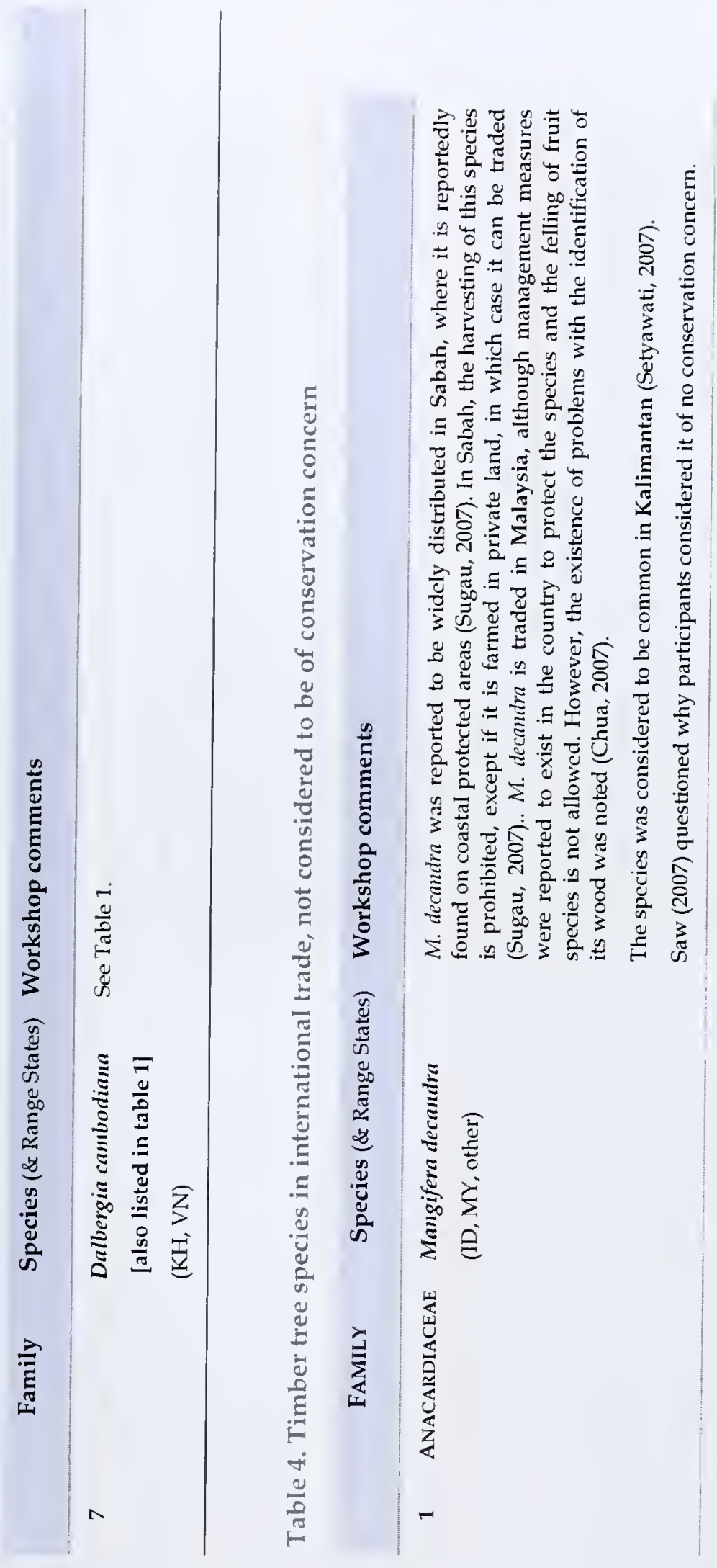




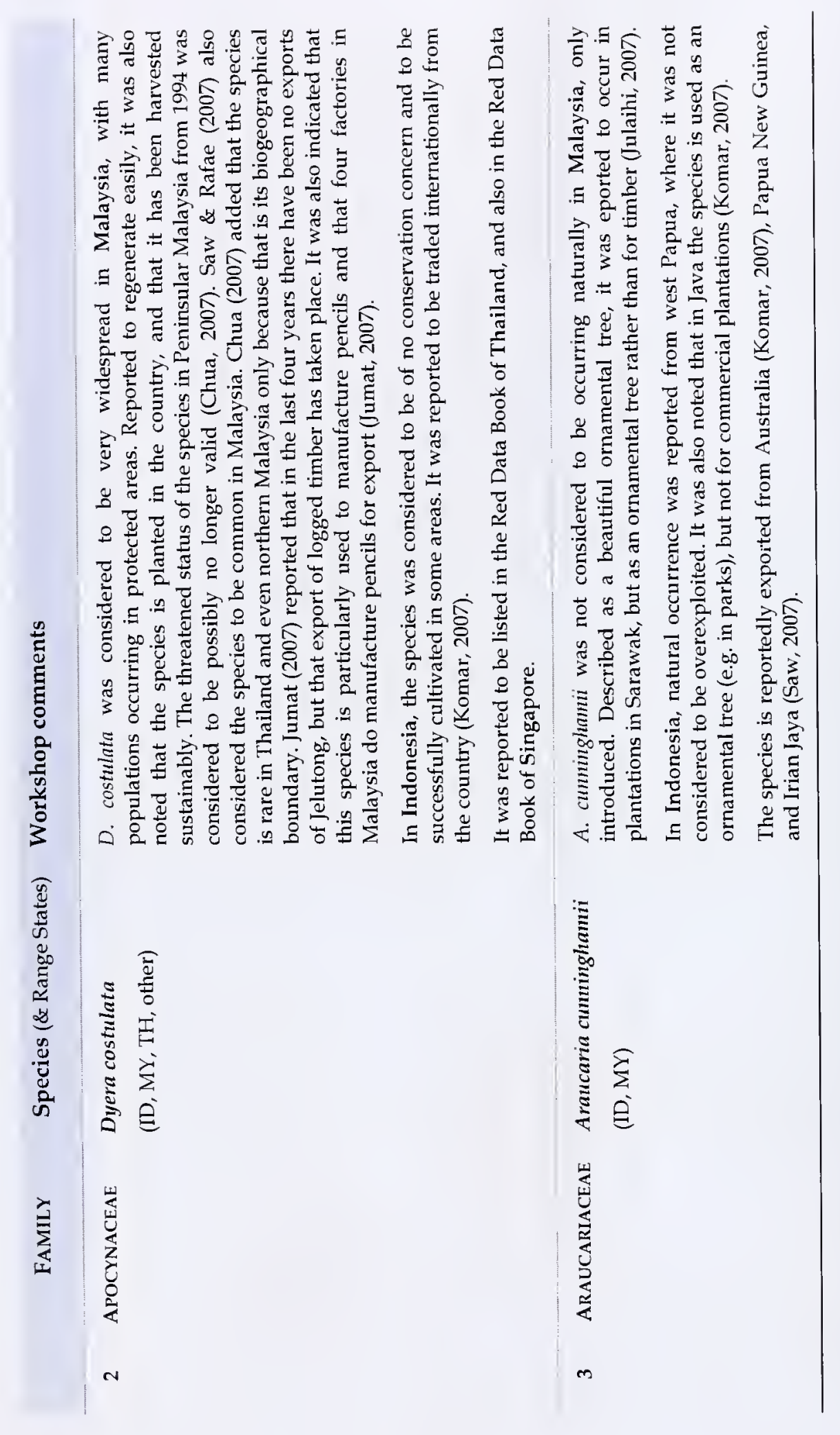

เก 


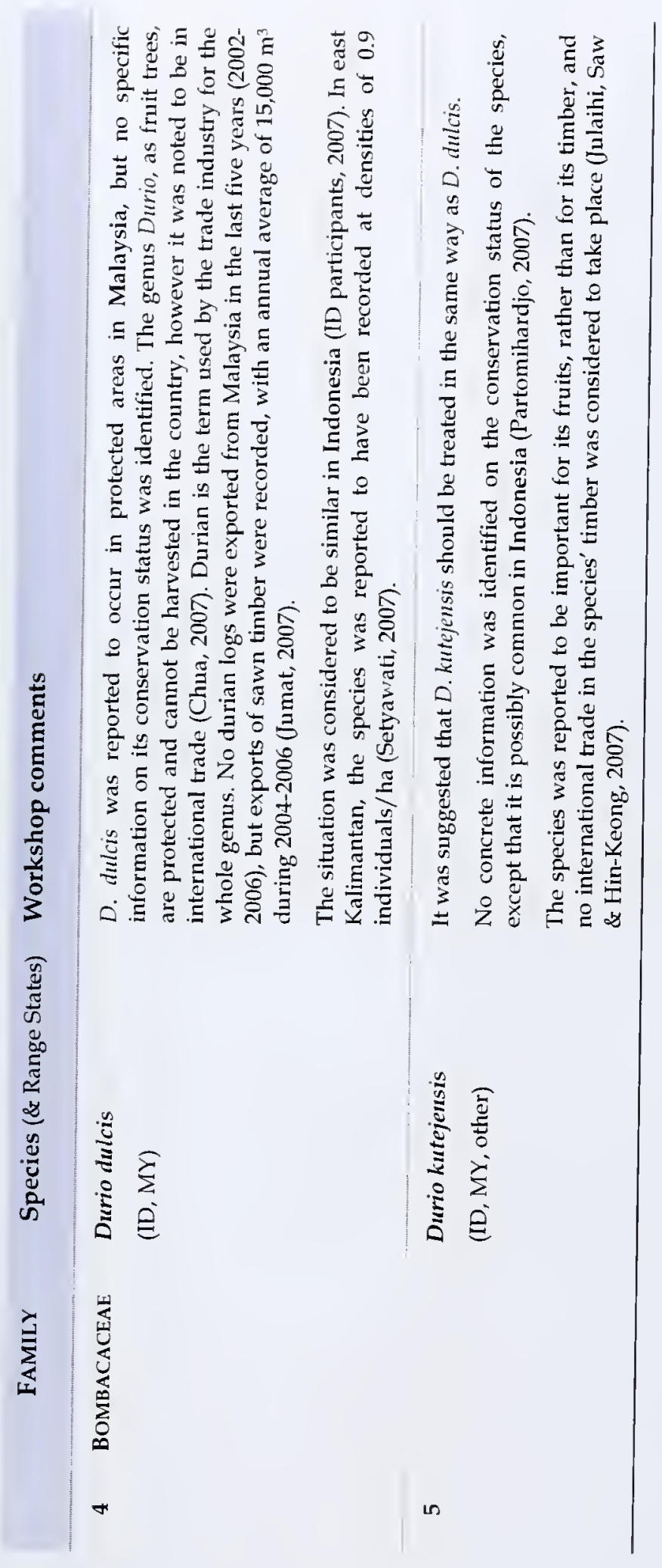




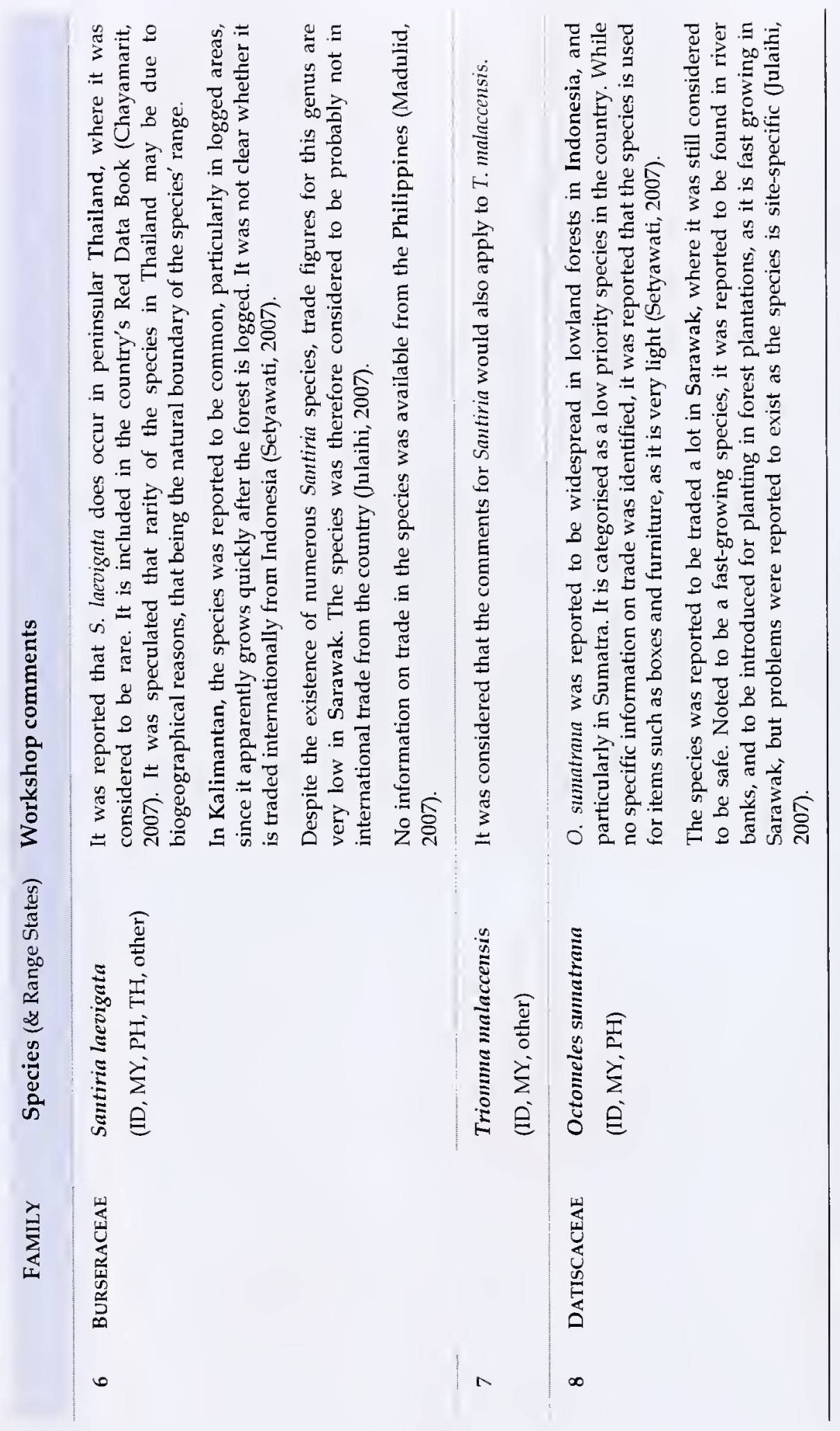

i̊ 


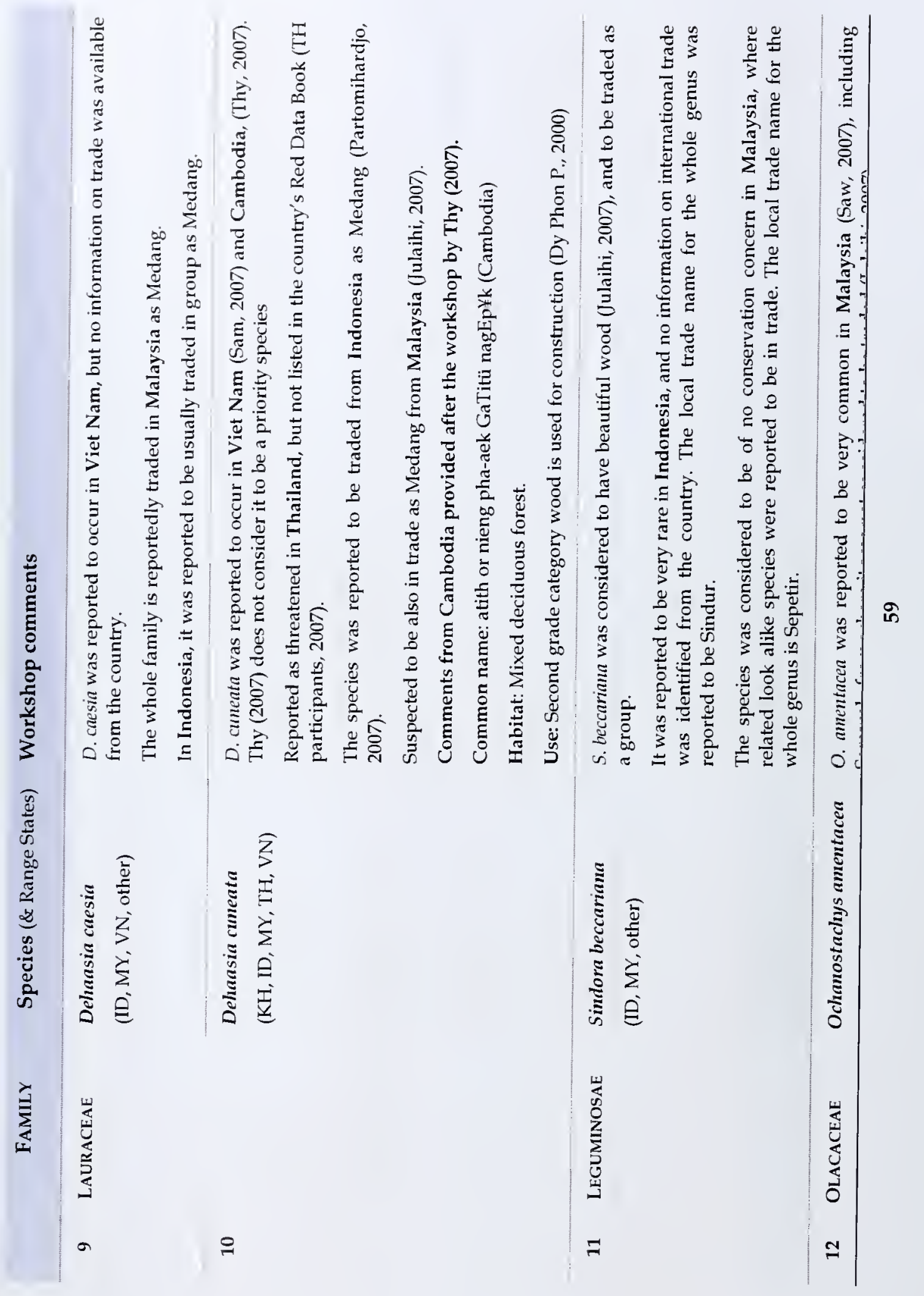




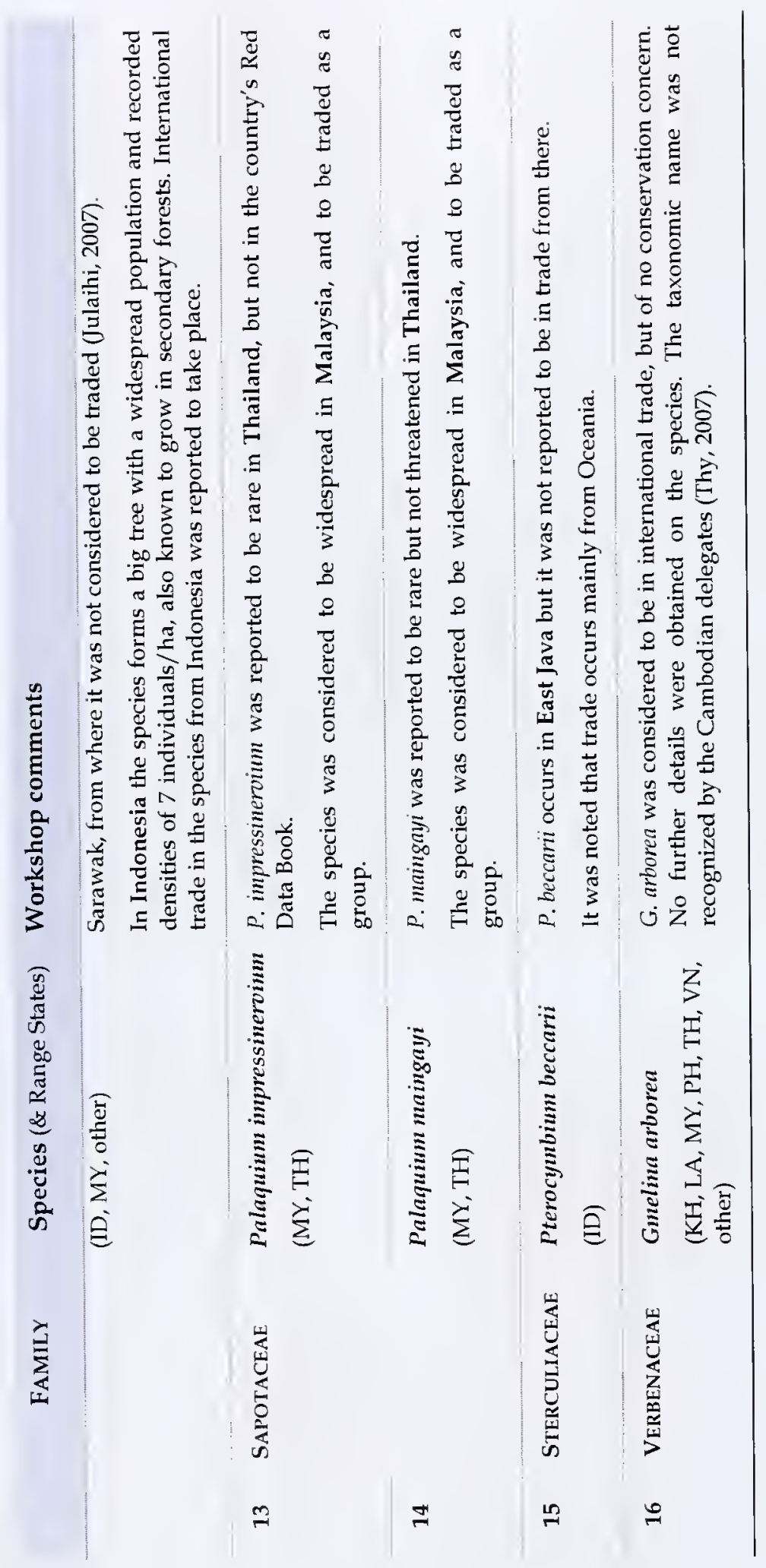

8 


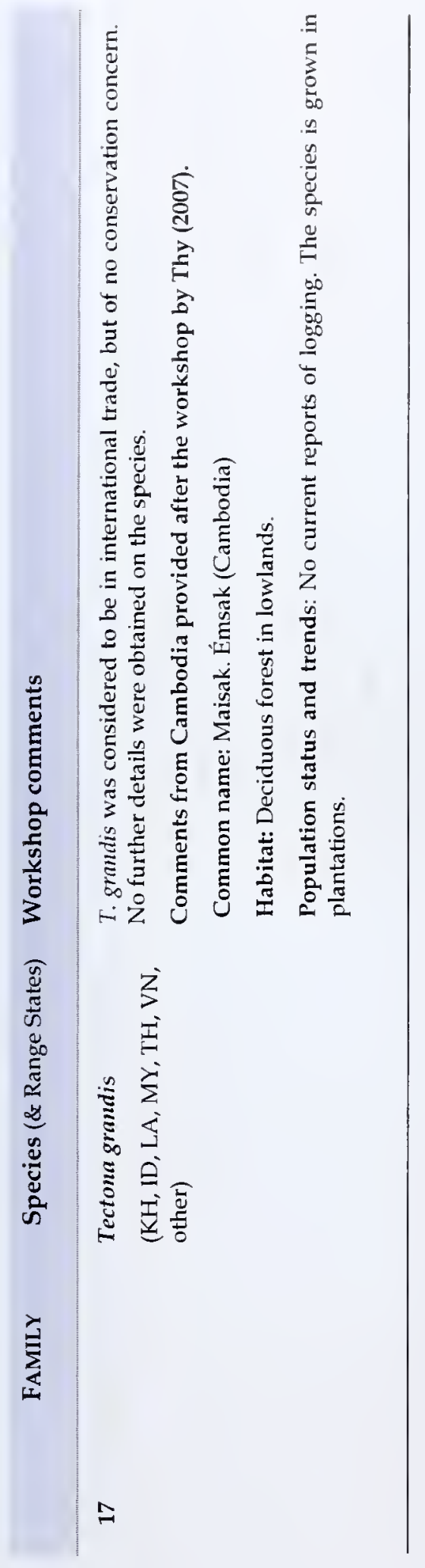




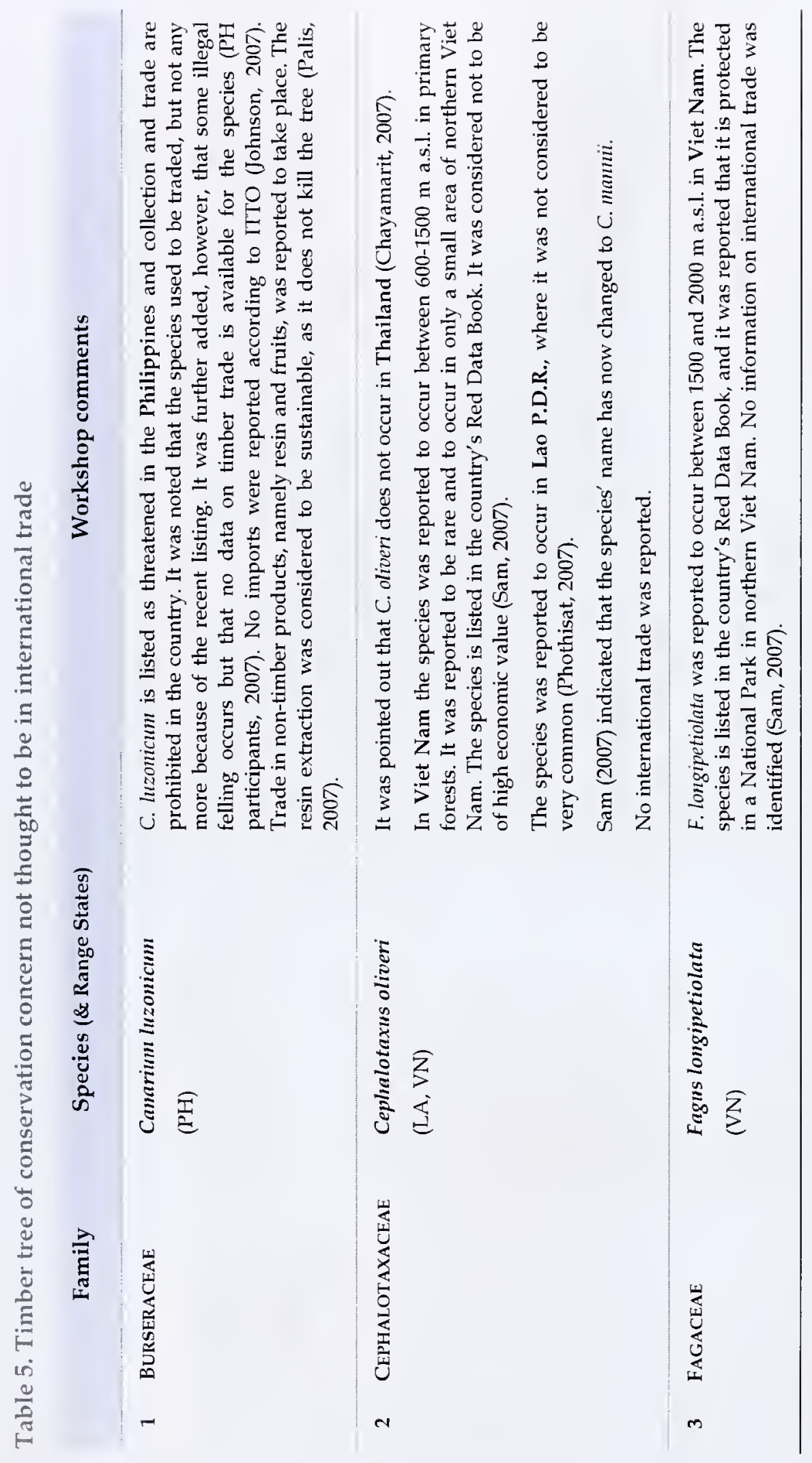









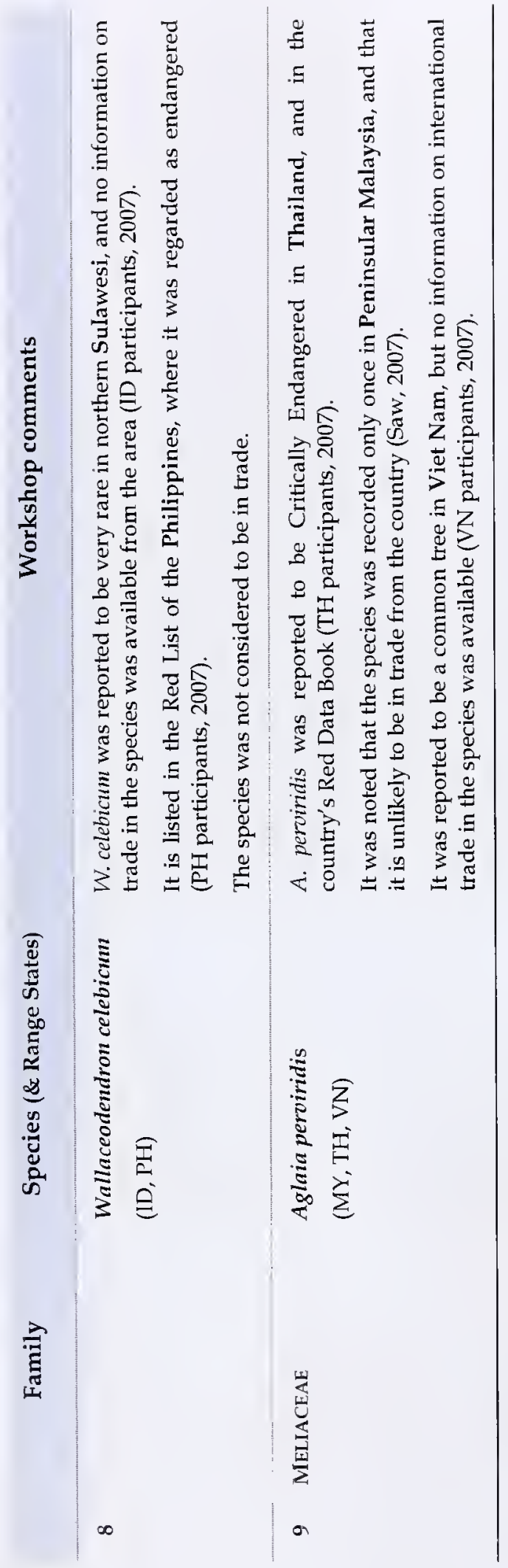




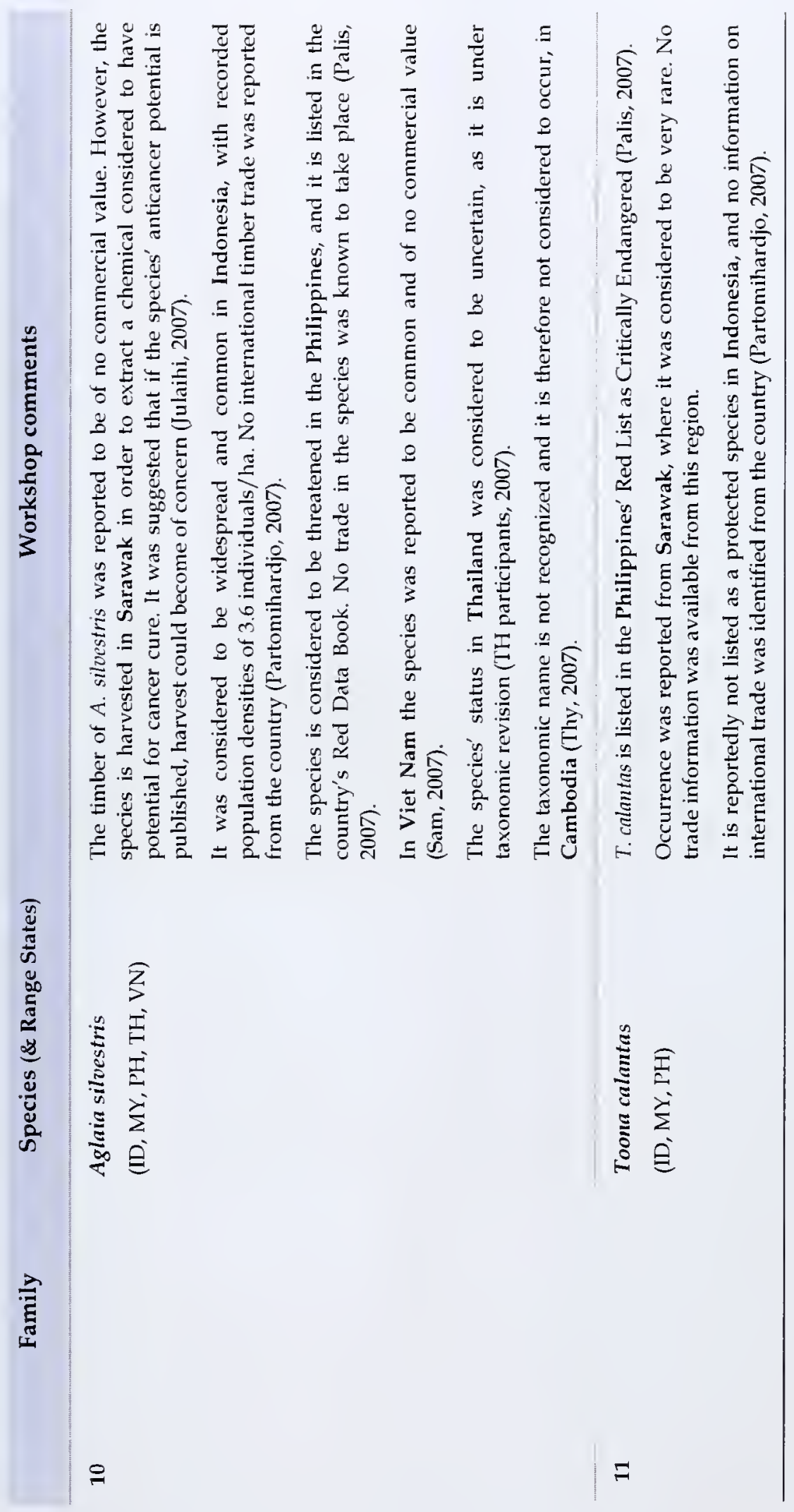




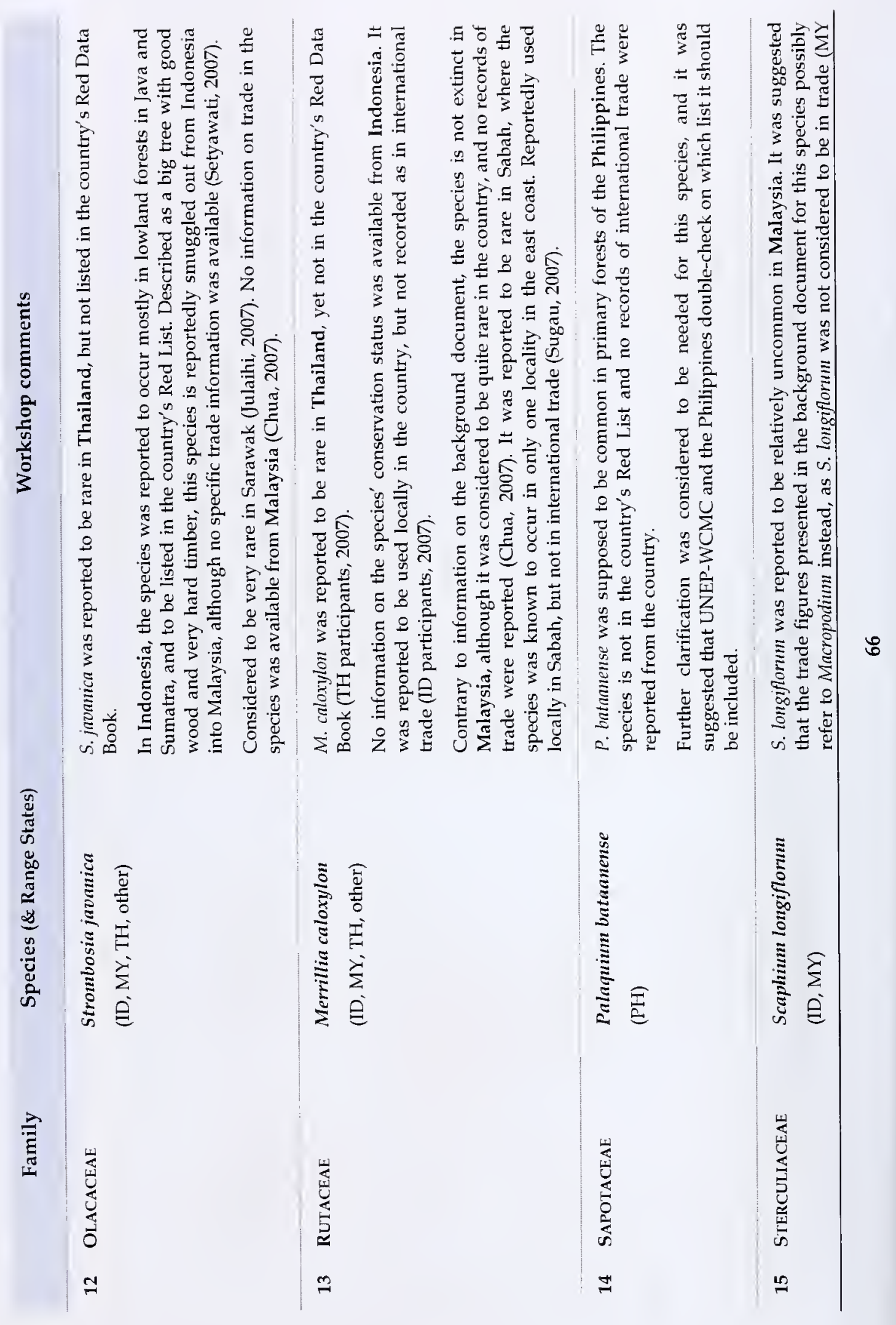




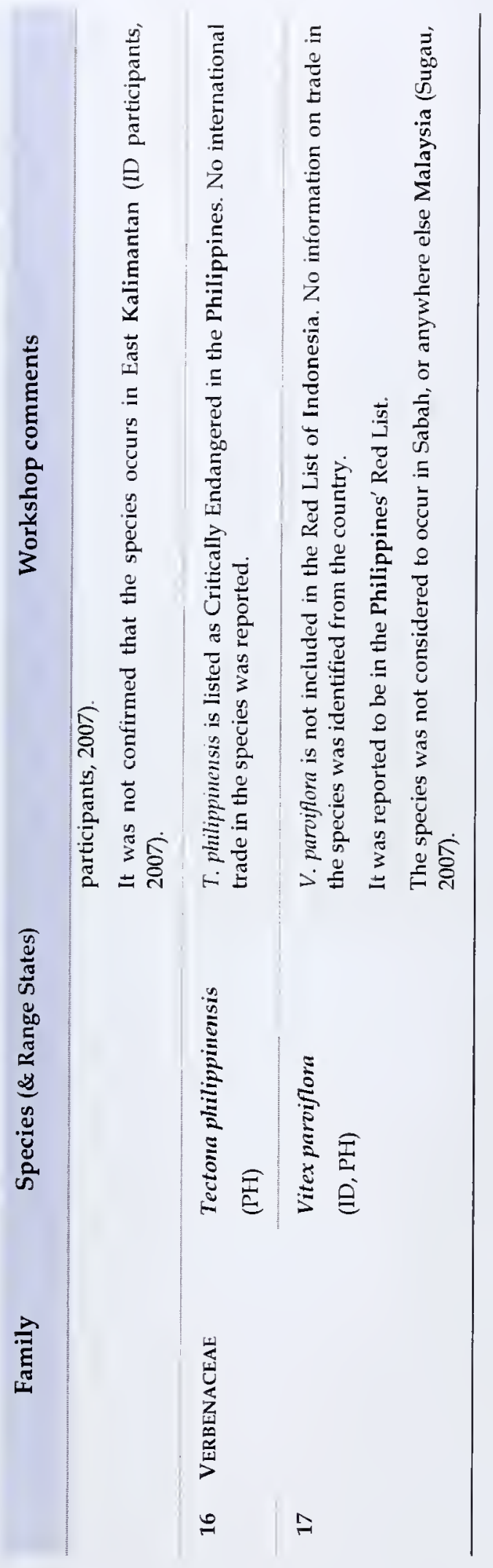




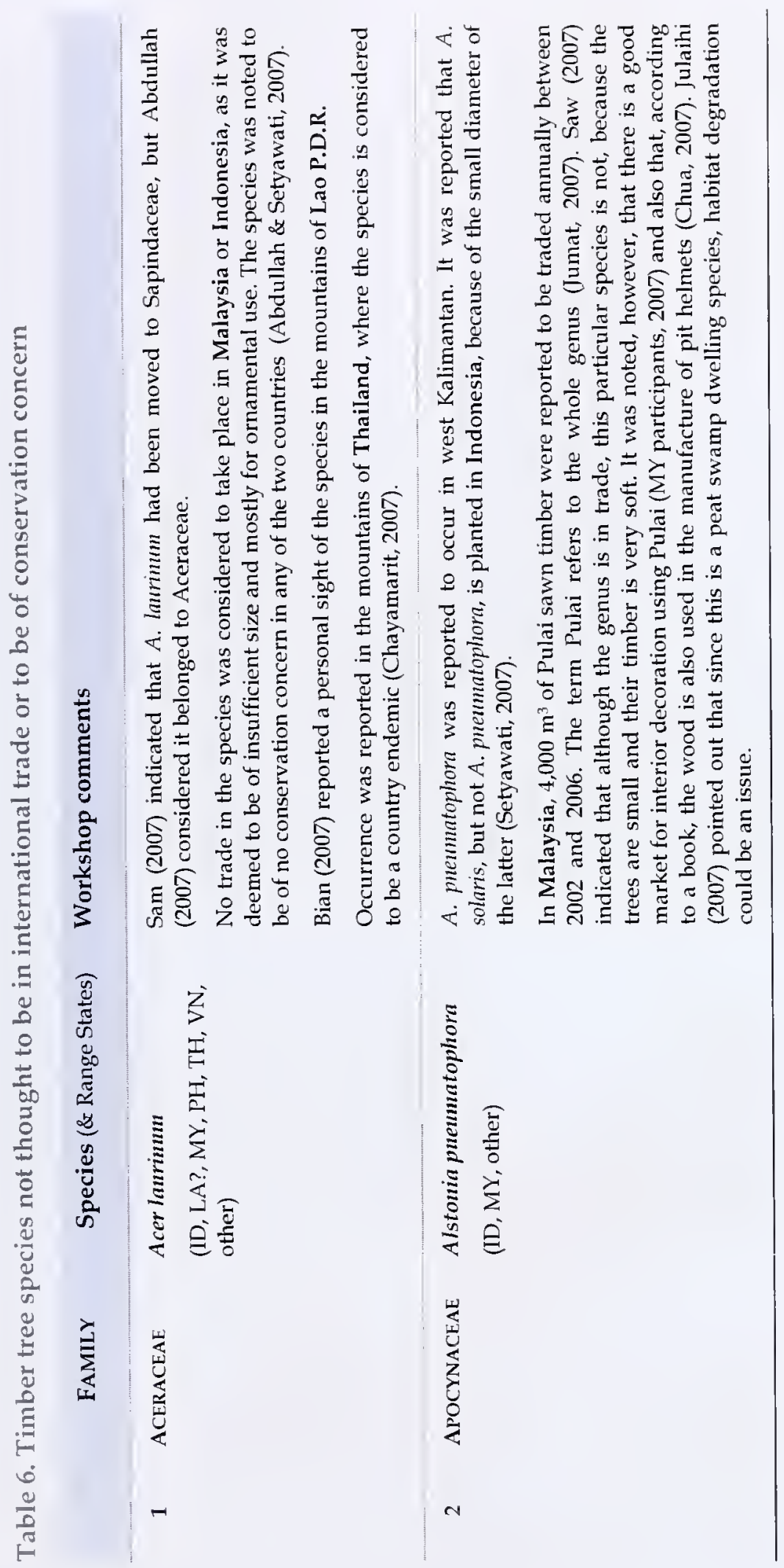




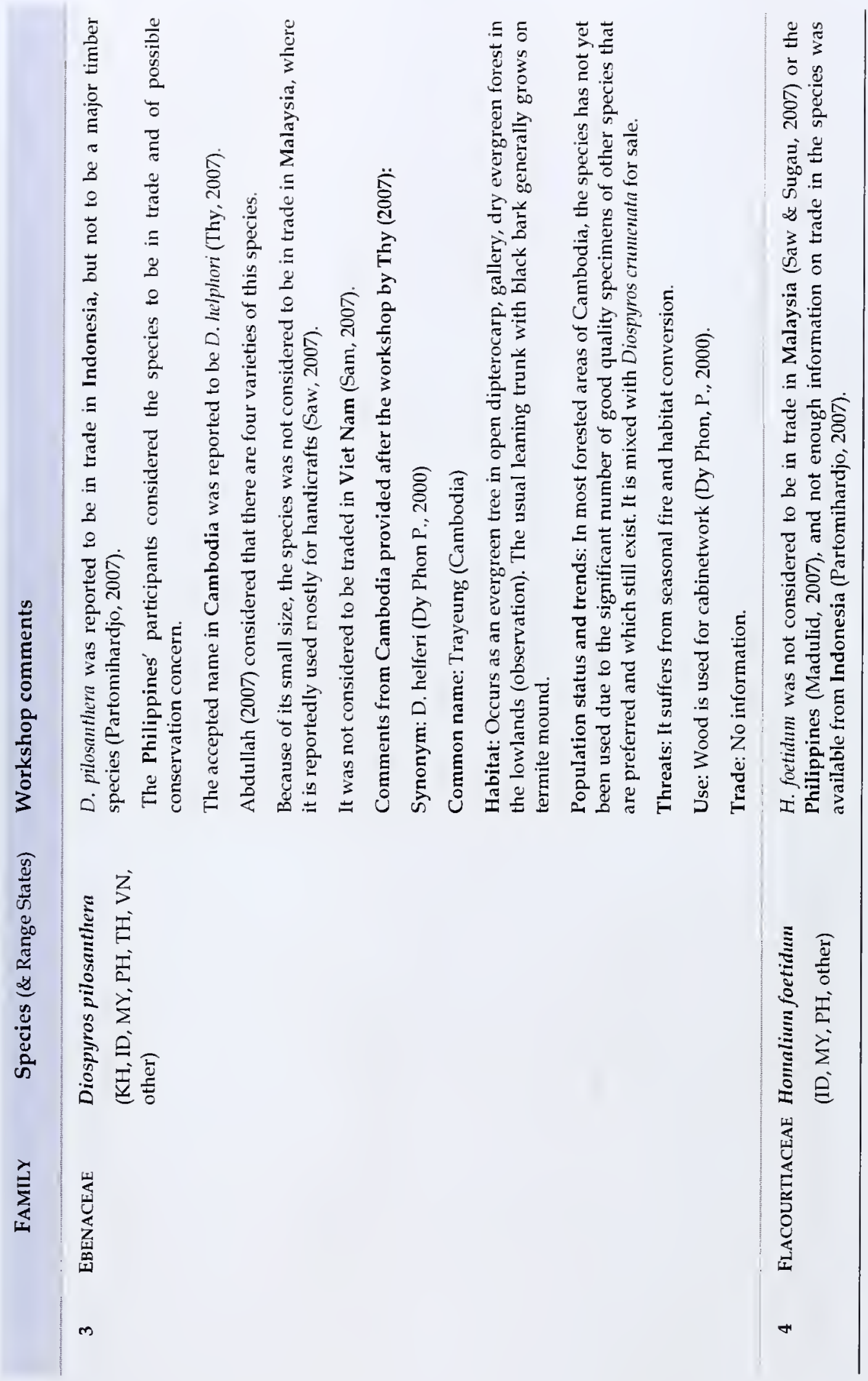




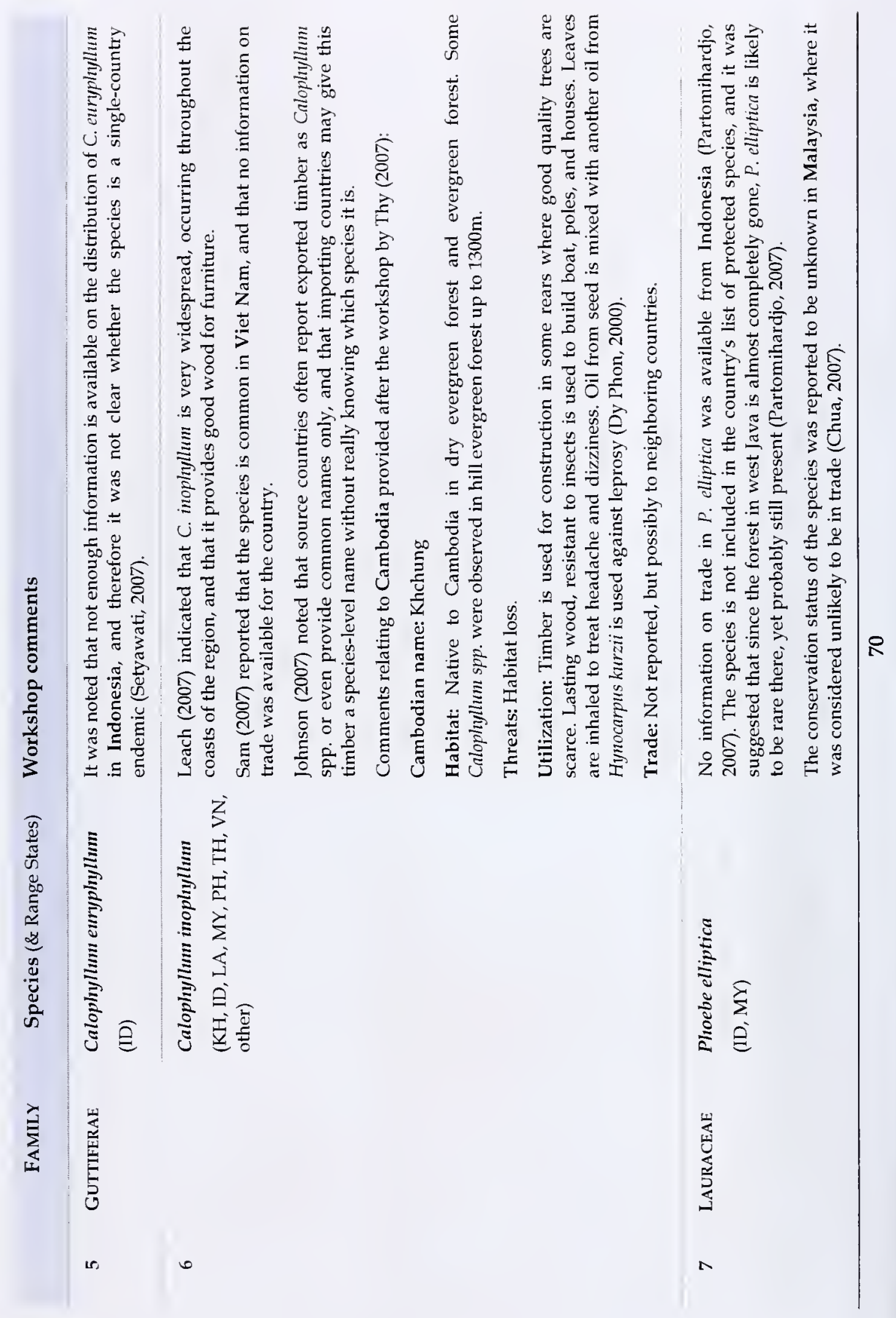




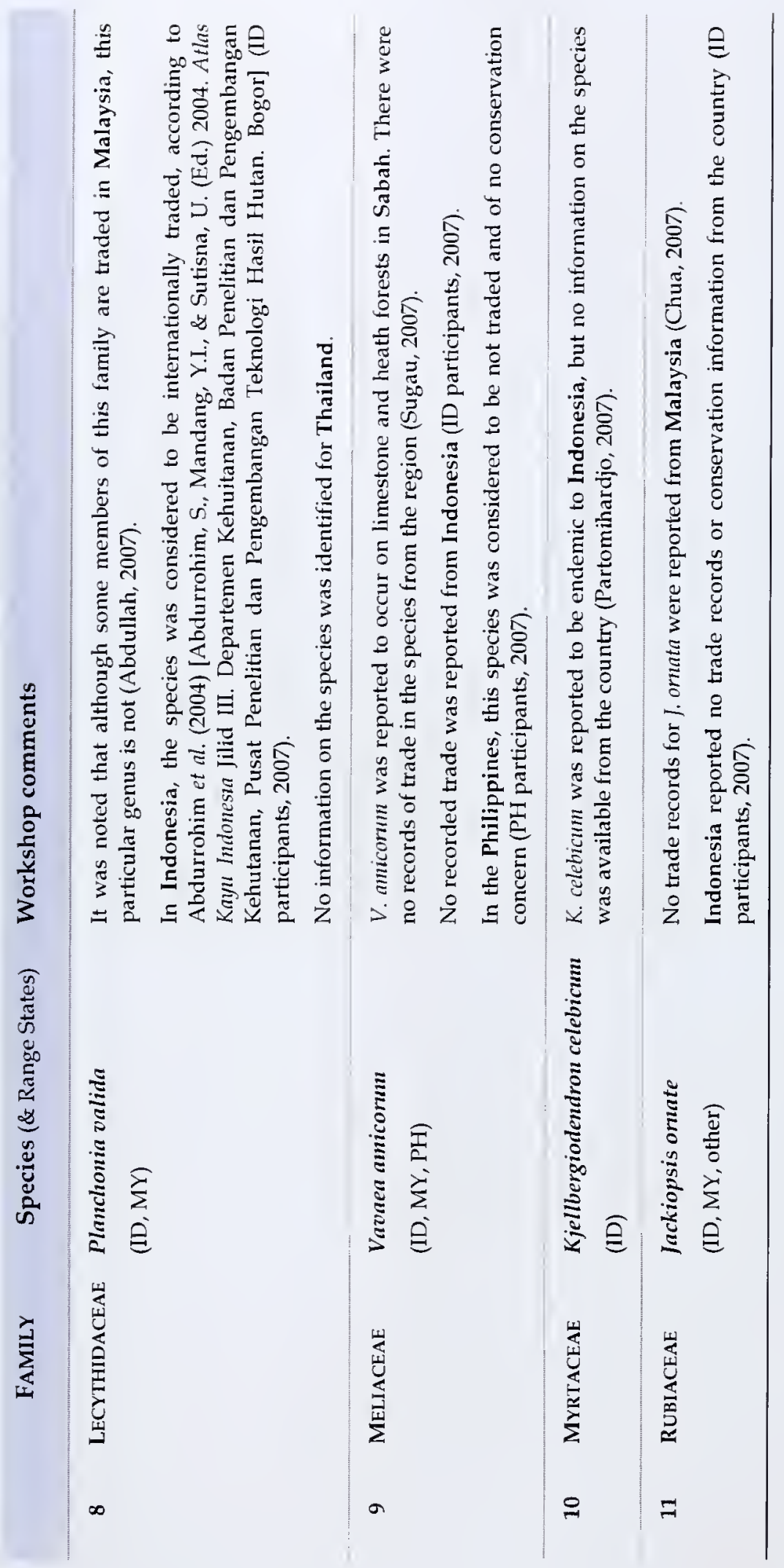

$F$ 


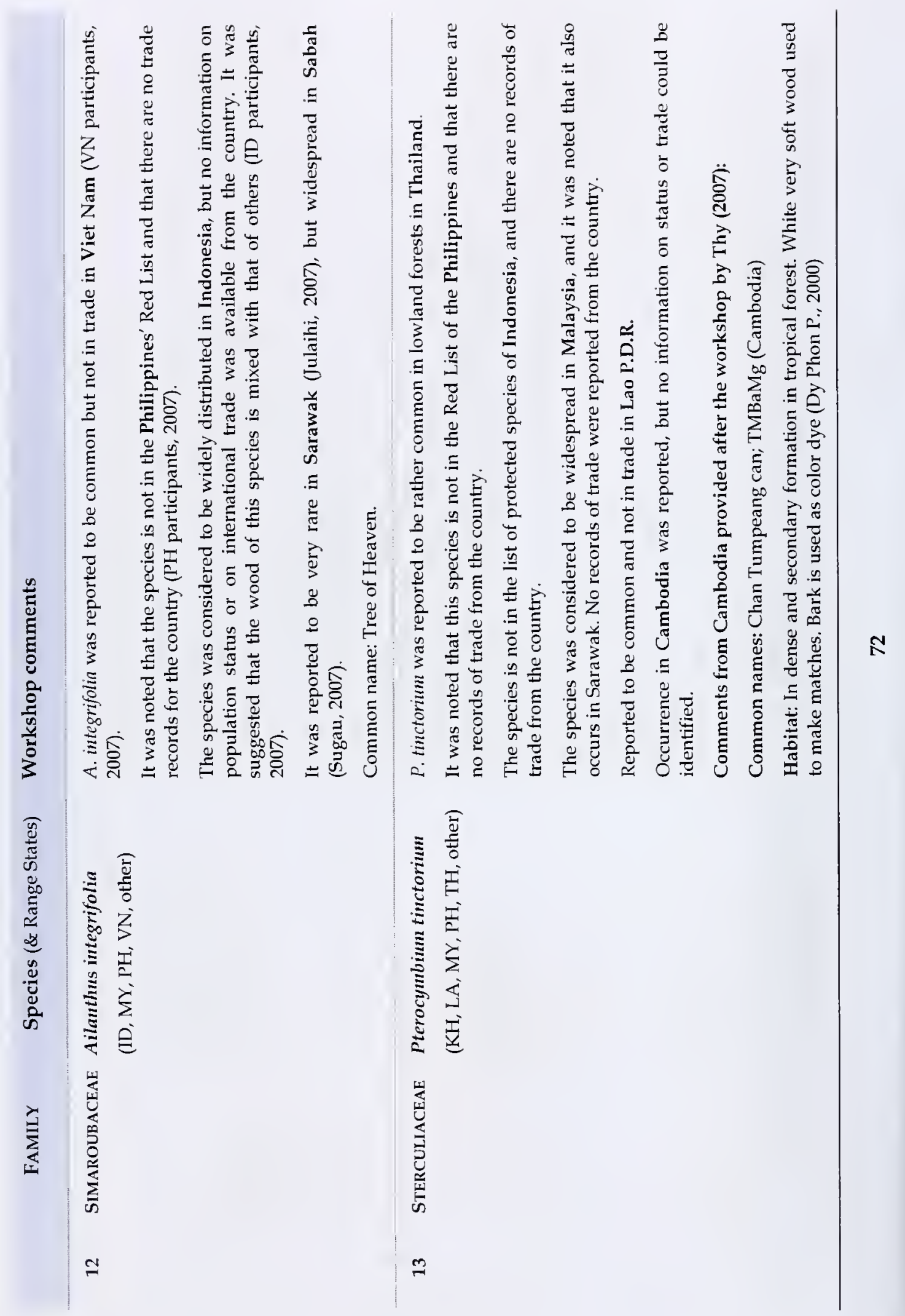




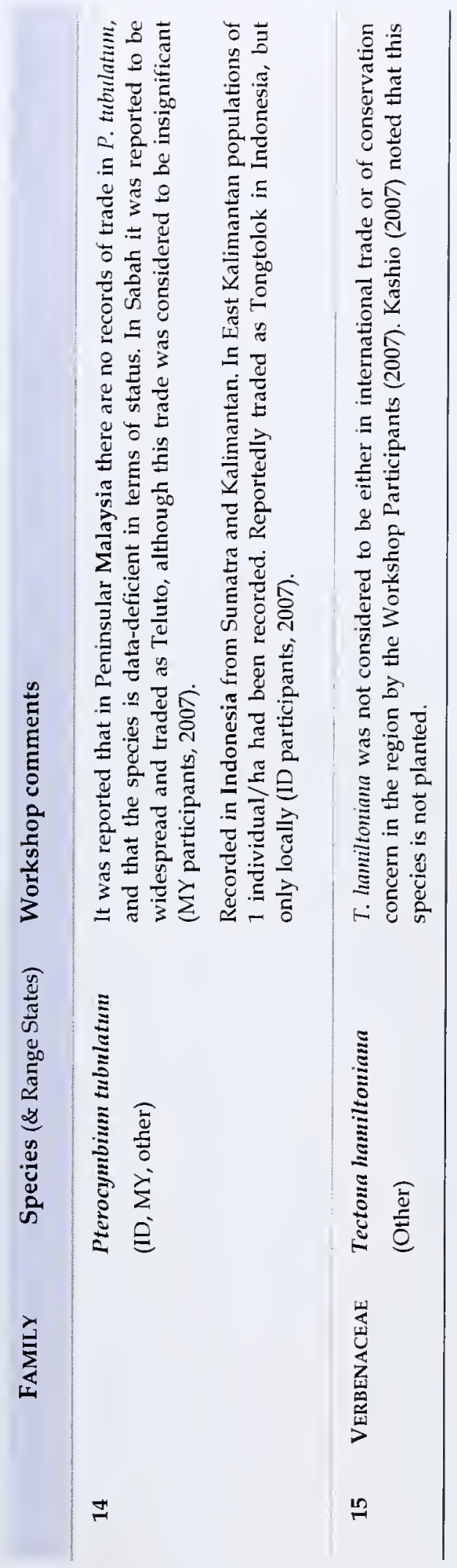


I 

